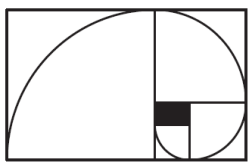

2011, Volume 20, 1-115

https://doi.org/10.21711/217504322011/em201

\title{
Breve introducción a la Matemática de la Estadística Espacial
}

\author{
Oscar Bustos y Aureliano Guerrero
}

\begin{abstract}
Spatial statistical techniques and geostatistics are applied in numerous disciplines, including: Biology, Economic Geography, Image Processing, Earth Sciences and Environment, Ecology, Geography, Epidemiology, Agronomy, Forestry, mineral prospecting, etc. These notes are an introduction to this subject from a mathematically rigorous point of view but without going in details into any particular topic. Chapter 1 gives a brief motivation to the subject by means of an example of the statistical analysis of satellite images. Chapter 2 is devoted to spatial models defined by processes with finite second order moments. Chapter 3 deals with Gibbs-Markov random fields defined on pairs of integers. A few concepts developed in Statistical Physics are adapted to image processing. Finally Chapter 4 discusses briefly aspects related to spatial correlation.
\end{abstract}





\section{Índice general}

1 Prólogo

2 Modelos Espaciales de Segundo Orden y Geoestadística 10

2.1 Conceptos y Resultados Básicos . . . . . . . . . . . . . 10

2.2 Funciones de covarianza definidas positivas y procesos gaus-

sianos . . . . . . . . . . . . . . . . 14

2.3 Estacionaridad estricta. Isotropía . . . . . . . . . . . . 16

2.4 Movimientos Brownianos . . . . . . . . . . . . . . . . . 17

2.5 Procesos intrínsecos y variogramas . . . . . . . . . . 18

2.6 Variogramas para procesos estacionarios . . . . . . . . 20

2.7 Ejemplos de covarianzas y variogramas . . . . . . . . . . . 21

2.8 Anisotropía . . . . . . . . . . . . . . . . . . . 22

2.9 Propiedades Geométricas: continuidad y diferenciabilidad . 23

2.10 Continuidad y diferenciabilidad en el caso estacionario . . . . . . . . . . . . . . . . . . 24

2.11 Representación espectral de covarianzas . . . . . . . . . 26

2.11 .1 Caso $S=\mathbb{R}^{2} \ldots \ldots \ldots \ldots \ldots$

2.11 .2 Caso $S=\mathbb{R} \ldots \ldots \ldots \ldots \ldots \ldots \ldots$

2.11 .3 Caso $S=\mathbb{Z}^{2} \ldots \ldots \ldots \ldots \ldots \ldots \ldots$

2.12 Modelos Autorregresivos Espaciales . . . . . . . . . . . . 28

2.12 .1 Modelos ARMA . . . . . . . . . . . . . . . . . 28

2.13 Procesos SAR (Simultáneos AR) . . . . . . . . . . . . 29

2.14 Procesos autorregresivos condicionales estacionarios . . . . 31

2.14.1 Ejemplos de aplicación de la Proposición 2.14.14 . 37

2.15 Modelos autorregresivos no-estacionarios sobre redes finitas 39

2.15.1 Representación SAR-local uniparamétrica . . . . . . 40

2.15.2 Representación CAR-Markov . . . . . . . . . . . . . 41

2.15 .3 Procesos de Gauss-Markov . . . . . . . . . . . . . 41

2.15.4 Grafo asociado a modelo SAR . . . . . . . . . . . . 43

2.16 Modelos de Regresión Espacial . . . . . . . . . . . . . . 44

2.17 Predicción con varianza conocida . . . . . . . . . . . . 46

2.17 .1 Kriging Universal . . . . . . . . . . . . . . . . 47 
3 Campos Aleatorios de Gibbs Markov sobre Redes 48

3.1 Potenciales y distribuciones de Gibbs . . . . . . . . . . . . . 49

3.2 Ejemplos de Potenciales y distribuciones de Gibbs . . . . . 55

3.3 Potenciales Normalizados e Identificables . . . . . . . . . . 59

3.3.1 El potencial $\Phi^{a}$ para distintos ejemplos . . . . . . . 70

3.4 Potenciales invariante por traslaciones . . . . . . . . . . 72

3.5 Auto-modelos de Besag . . . . . . . . . . . . . . . 73

3.6 Ejemplos de auto-modelos $\mathcal{G}$-markovianos . . . . . . . . . . 77

4 Inferencias en Modelos Espaciales $\quad \mathbf{8 2}$

4.1 Estimación en Geoestadística . . . . . . . . . . . . . . . . . 82

4.1 .1 El caso isotrópico . . . . . . . . . . . . . 86

4.2 Estimación paramétrica en el caso isotrópico fijando $k$ distancias: $0<r_{1}<\ldots<r_{k}(k<\infty) \ldots \ldots$. . . . . 87

4.2.1 Estimador de mínimos cuadrados generalizado . . . 89

4.2.2 Estimador de mínimo contraste . . . . . . . . . . . 89

4.3 Estimación de la función semivariograma bajo presencia de

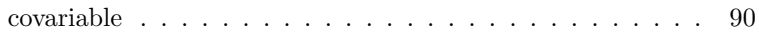

4.4 Estimación de Máxima Verosimilitud . . . . . . . . . . . . . 91

4.5 Validación de modelos para función de semivariograma en el caso isotrópico . . . . . . . . . . . . . . . . . . . 91

4.5.1 Validación cruzada de $H_{0}$. . . . . . . . . . . . . . . 91

4.5.2 Validación de $H_{0}$ por "bootstrap" paramétrico . . . 93

4.6 Autocorrelación en redes espaciales . . . . . . . . . . . . . . 94

4.6 .1 Índice de Moran . . . . . . . . . . . . . . . . . 95

4.6.2 Test asintótico de independencia espacial . . . . . . 98

4.6.3 Cálculo exacto de la esperanza y varianza del índice

de Moran bajo normalidad . . . . . . . . . . . . . . . . 99

4.6 .4 Índice de Geary . . . . . . . . . . . . . . . . . . . 108

4.6.5 Cálculo exacto de la esperanza y varianza del índice de Geary bajo normalidad . . . . . . . . . . . 108

Bibliografía 


\section{Capítulo 1}

\section{Prólogo}

Las técnicas de Estadística Espacial y Geoestadística tienen un rápido desarrollo en estos tiempos, posiblemente a causa del amplio campo de su aplicabilidad. En efecto, es posible encontrarlas en trabajos de numerosas disciplinas, entre otras: Biología, Geografía Económica, Procesamiento de Imágenes, Ciencias de la Tierra y del Medio Ambiente, Ecología, Geografía, Epidemiología, Agronomía, Recursos Forestales, Prospección de Minerales, etc. Referencias a algunos de esos trabajos pueden verse por ejemplo en www.inpe.br y www.conae.gov.ar.

Esa diversidad de aplicaciones y situaciones hace de la Geoestadística una disciplina de gran riqueza y un amplio campo todavía poco explorado por la investigación tanto en Matemática como en Estadística.

Estas notas no pretenden ser más que una introducción al tema desde un punto de vista matemáticamente riguroso pero sin profundizar en tema alguno en particular. Fueron escritas con la atención puesta en dos libros muy recomendables para quienes deseen profundizar en los temas tratados e ir más allá todavía en importantes temas aquí no tratados como los que tienen que ver con Entropía, Grandes Desvíos, Métodos Variacionales, etc. Esos libros son: Gaetan and Guyon (2010) ([5]) y Georgii (1988) ([6]).

Cuando realizamos un análisis de datos debemos buscar un modelo que se ajuste a tales datos.

Por ejemplo, si queremos conocer la distribución de la variable altura, $H$, de personas adultas en un cierto país, tomaremos varias personas adultas, digamos $n$, entre los habitantes de ese país y mediremos su altura obteniendo datos $h_{1}, \cdots, h_{n}$. En los cursos de Estadística Básica hemos aprendido que en un caso como este, es razonable considerar que esos valores son realizaciones (valores observados) de variables aletaorias $H_{1}, \cdots, H_{n}$ que son independientes (e idénticamente distribuidas como $H$ ).

Ahora si queremos conocer la distribución de los valores de energía, $X$, provenientes de distintos puntos de una cierta región geográfica como la 
que muestra la Figura 1, por medio de instrumentos adecuados,

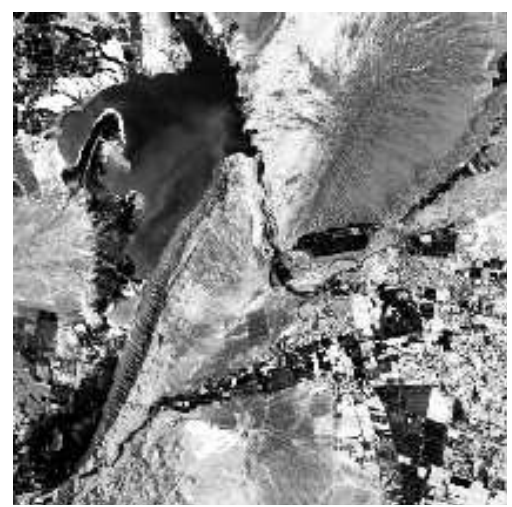

Figura 1. Dique de Ullún

San Juan (Argentina)

podemos trazar una grilla sobre la Figura 1,

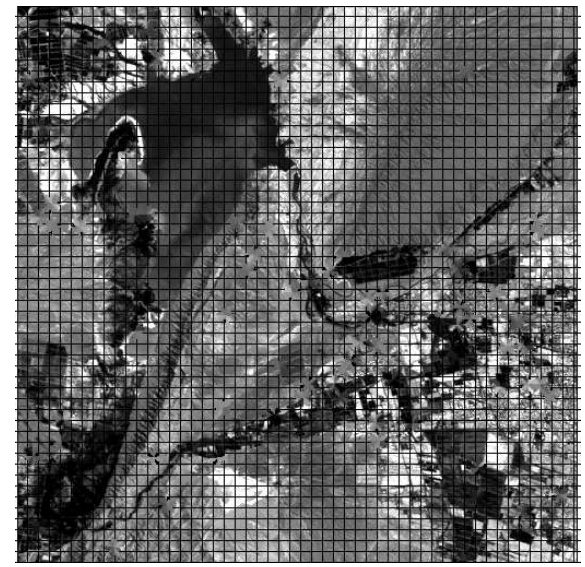

Figura 2. Grilla sobre Figura 1 
digamos de $n$ columnas y $m$ filas, y a cada celda de la misma asignar un cierto valor representativo del promedio de la energía proveniente de esa celda, obteniendo así la matriz de datos

$$
x=\left[\begin{array}{ccc}
x_{(1,1)} & \cdots & x_{(n, 1)} \\
\cdots & \cdots & \cdots \\
x_{(1, m)} & \cdots & x_{(n, m)}
\end{array}\right] .
$$

(En imágenes se acostumbra considerar al primer índice como el correspondiente a la columna y el segundo a la fila, esto es: $x_{(i, j)}$ valor de la matriz $x$ en la celda ubicada en la columna $i$ y la fila $j$ ). También aquí podemos considerar que cada $x_{(i, j)}$ es la realización de una variable aleatoria $X_{(i, j)}$. pero, a diferencia del ejemplo anterior, aquí no es razonable suponer que $X_{(1,1)}, \cdots, X_{(n, m)}$ son independientes.

Situaciones donde las variables aleatorias trabajadas no pueden considerarse como independientes se presentan en Series de Tiempo. Por ejemplo si estudiamos la distribución de la temperatura a lo largo del tiempo en una cierta ciudad consideramos una sucesión $T_{1}, \cdots, T_{n}$ de variables aleatorias con cierta estructura de correlación entre ellas que representa la relación entre la temperatura en el instante $t$ con la temperatura en instantes anteriores en el tiempo: $t-1, t-2, \ldots$ En este tema son muy populares los llamados modelos autoregresivos (modelos $\mathbf{A R}(\mathbf{p})$ ):

$$
T_{t}=\phi_{1} T_{t-1}+\cdots+\phi_{p} T_{t-p}+\varepsilon_{t}, \quad t=p+1, \cdots, n
$$

donde $\phi_{1}, \cdots, \phi_{p}$ son parámetros a estimar. En este caso se suele decir que consideramos un modelo causal, en el sentido que el valor de la variable $T_{t}$ en cierta forma está "causado" por los valores de $T_{s}$ con $s \leq t-1$.

Ahora, en el caso de los datos referidos al ejemplo del dique de Ullún: familias de variables aleatorias cuyos índices se refieren a posiciones espaciales, no tiene sentido suponer que la influencia de otras variables en $X_{(i, j)}$ deba considerarse restringida a $X_{\left(i^{\prime}, j\right)}$ con $i^{\prime} \leq i$ o a $X_{\left(i, j^{\prime}\right)}$ con $j^{\prime} \leq j$. En efecto, la originalidad de la Estadística Espacial (o Geoestadística como algunos prefieren) es incorporar en el modelo a ser ajustado a las observaciones el concepto de no-causalidad.

Extendiendo los modelos AR(p) en Estadística Espacial se consideran los llamados modelos AR 2D. En nuestro trabajo estos modelos son estudiados en el Capítulo 2. Son numerosos los trabajos donde se muestran las ventajas que se obtienen con estos modelos para muchas de las operaciones habituales en procesamiento de imágenes: filtrado de ruido, segmentación, clasificación, etc. 
Simplemente a manera de ejemplo. En la Figura 3

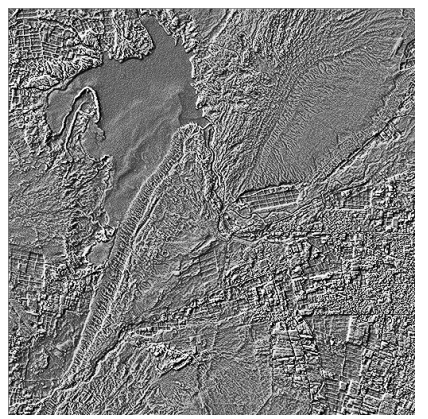

Figura 3. Segmentación de imagen en Figura 1.

se puede observar una segmentación (y detección de bordes) en la imagen de la Figura 1 obtenida por medio del ajuste de un proceso AR 2D a los datos de $X$, aplicando el algoritmo presentado en el trabajo de Ojeda et al (2010) ([9]).

Estos modelos AR 2D (o más generalmente ARMA 2D) son utilizados cuando trabajamos con variables cuyo rango es todo un intervalo de la recta. Pero no se deben aplicar cuando tenemos datos discretos como las imágenes de clase donde el valor de cada $X_{(i, j)}$ está reducido a un conjunto de "etiquetas", por ejemplo:

\{suelo desnudo, vegetación, agua, urbano\} .

Para llevar en cuenta la correlación de cada variable con sus vecinos espaciales en tal situación se vienen usando desde hace ya algunos años (ver por ejemplo Besag (1989) ([2])) los llamados procesos de Markov que son extensiones de las Cadenas de Markov muy usadas en diversos contextos.

Más adelante (3.2.3) veremos uno de los más simples y usados procesos de Markov: el Modelo de Ising. En Frery et al. (2009) ([4]) se puede ver el comportamiento de este modelo para lograr clasificaciones en imágenes, más exactas y eficientes que las obtenidas con las técnicas usuales de clasificación que se aplican en el caso de variables independientes.

Desde el punto de vista de la Estadística Matemática la extensión de resultados referidos a convergencia en probabilidad, en distribución, teoremas de ergodicidad, etc. de modelos causales a no-causales es un verdadero desafío. En la mayoría de los casos es preciso estudiar nuevos conceptos, resultados específicos para estos modelos no-causales y no siempre es posible extender resultados que valen para modelos causales a estos últimos. 
El Capítulo 2 está dedicado a modelos espaciales definidos por procesos con momento de segundo orden finito y propiedades adicionales cuando así se requiera. No se dan pruebas de los resultados a fin de no extendernos en demasía. Por otra parte esas demostraciones son en su mayoría bien conocidas y pueden encontrarse en el ya citado Gaetan and Guyon (2010) $([5])$.

En Capítulo 3 nos referimos a Campos Aleatorios de Gibbs - Markov sobre redes definidas por pares de enteros. Aquí preferimos dar las demostraciones pues son menos conocidos los resultados de este Capítulo que los del anterior. Se trata de adaptar varios conceptos desarrollados en Física Estadística al procesamiento de imágenes.

Por último en el Capítulo 4 estudiamos brevemente los aspectos referidos a la correlación espacial. He aquí como dijimos anteriormente la principal distinción entre la Estadística Espacial y la Estadística como se la necesita para otras aplicaciones.

Esperamos que este pequeño texto pueda servir para entusiasmar a un amante de la Matemática para adentrarse en el campo apenas explorado, bello y rico en aplicaciones, de la Estadística Espacial. 


\section{Capítulo 2}

\section{Modelos Espaciales de Segundo Orden y Geoestadística}

\subsection{Conceptos y Resultados Básicos}

Sean $(\Omega, \mathcal{F}, P)$ un espacio de probabilidad, $S \subseteq \mathbb{R}^{2}$ no vacío, $(E, \mathfrak{E})$ un espacio medible.

Definición 2.1.1. Una imagen con soporte en $S$ y espacio de estado E es una función $x: S \longmapsto E$.

Notación 2.1.2. Usaremos $E^{S}$ para representar al conjunto de todas las imágenes con soporte en $S$.

Notación 2.1.3. Si $F$ es un conjunto y $\mathcal{G}$ es una familia no vacía de subconjuntos de $F$ con $\sigma(\mathcal{G})$ designaremos a la $\sigma$-álgebra de $F$ generada por $\mathcal{G}$.

Ejemplo 2.1.4. Ejemplos:

1. Imagen binaria: $E$ con dos elementos: Por ejemplo $E:=\{0,1\}$.

2. Imagen en escala de grises: $E=\{0,1, \ldots, 255\}$.

Ejemplo 2.1.5. 1. En este caso, el significado visual es:

$0 \longleftrightarrow$ negro $\quad 255 \longleftrightarrow$ blanco.

2. Imagen a colores: $E=\{0,1, \ldots, 255\}^{3}=\{(a, v, r) / 0 \leq a, v, r \leq 255\}$. 
3. Imagen multiespectral: $E=\{0,1, \ldots, 255\}^{k}$ con $k \geq 3$ ( $k$ es el número de bandas).

Definición 2.1.6. Llamaremos proceso estocástico de imágenes (definido sobre $\Omega$ con soporte $S$ y espacio de estados E) a

$$
X:=\left\{X_{s} / s \in S \text { y } X_{s}: \Omega \longmapsto E \text { es }(\mathcal{F}, \mathfrak{E}) \text { medible }\right\} .
$$

Si $\omega \in \Omega$, llamaremos trayectoria de $X$ a la función $X_{\omega}: S \longmapsto E$ (imagen con soporte en $S$ y espacio de estados E) dada por

$$
X_{\omega}(s)=X_{s}(\omega)
$$

Ejemplo 2.1.7. $\quad$ 1. Si $\#(E)<\infty$, siempre tomaremos $\mathfrak{E}=\mathcal{P}(E) \quad($ la familia de todos los subconjuntos de $E$ ).

2. Si $E \subset \mathbb{R}^{k}$ con $k \geq 1$, pondremos $\mathfrak{E}=\left\{B \cap E / B \in \mathcal{B}_{k}\right\}$ donde $\mathcal{B}_{k}$ es la $\sigma$-álgebra de Borel de $\mathbb{R}^{k}$. En este caso, diremos que $(E, \mathfrak{E})$ es un espacio Euclideo si $E$ es un subespacio vectorial de $\mathbb{R}^{k}$.

3. El caso más general que trataremos es $E=$ espacio métrico. En tal caso, E será la $\sigma$-álgebra de Borel de E (esto es, la $\sigma$-álgebra generada por la familia de abiertos de $E$ ).

Definición 2.1.8. Sean $X_{1}=\left\{X_{1, s} / s \in S\right\}, X_{2}=\left\{X_{2, s} / s \in S\right\}$ y $\mathcal{F}\left(X_{i}\right)=$ $\sigma\left(\left\{X_{i, s}^{-1}(B) / s \in S, B \in \mathcal{B}\right\}\right)$. Se dice que $X_{1} y X_{2}$ son $P$ independientes si

$$
A \in \mathcal{F}\left(X_{1}\right) B \in \mathcal{F}\left(X_{2}\right) \Rightarrow P(A \cap B)=P(A) \cdot P(B) .
$$

Sean $X_{1}, \ldots, X_{n} n$ procesos con soporte $S_{1}, \ldots, S_{n}$ respectivamente. Se dice que son $P$-independientes si $\forall k \leq n$ y un conjunto de indices $1 \leq i_{1}<$ $\ldots<i_{k} \leq n$, si $A_{i_{1}} \in \mathcal{F}\left(X_{i_{1}}\right), \ldots, A_{i_{k}} \in \mathcal{F}\left(X_{i_{k}}\right) \Rightarrow P\left(A_{i_{1}} \cap \ldots \cap A_{i_{k}}\right)=$ $\prod_{j=1}^{k} P\left(A_{i_{j}}\right)$.

Definición 2.1.9. Sean $E=\mathbb{R} ; \mathfrak{E}=\mathcal{B}_{1} ; X=\left\{X_{s} / s \in S\right.$ y $X_{s}: \Omega \longmapsto E$ es $(\mathcal{F}, \mathfrak{E})-$ medible $\}$ un proceso estocástico de imágenes con soporte $S y$ espacio de estados E. Diremos que:

a) $X$ es de $1^{\circ}$ orden, si $X_{s} \in \mathcal{L}^{1}(\Omega, \mathcal{F}, P, \mathbb{R}) \forall s \in S$. En tal caso llamaremos a la función $\mu_{X}: S \longmapsto \mathbb{R}$ dada por $\mu_{X}(s):=E_{P}\left(X_{s}\right)$ media de $X$.

b) $X$ es de $\mathscr{2}^{\circ}$ orden, si $X_{s} \in \mathcal{L}^{2}(\Omega, \mathcal{F}, P, \mathbb{R}) \forall s \in S$. En tal caso:

- Llamaremos varianza de $X$, a la función $\operatorname{Var}_{X}: S \longmapsto \mathbb{R}$ dada por $\operatorname{Var}_{X}(s)=\operatorname{Var}_{P}\left(X_{s}\right)$. 
- Llamaremos covarianza de $X$, a la función $C_{X}: S \times S \longmapsto \mathbb{R}$ dada por $C_{X}(s, t)=E_{P}\left[\left(X_{s}-\mu_{X}(s)\right)\left(X_{t}-\mu_{X}(t)\right)\right]$.

- Llamaremos correlación de $X$, a la función $\rho_{X}: S \times S \longmapsto \mathbb{R}$ dada por $\rho_{X}(s, t)=\frac{C_{X}(s, t)}{\left(\operatorname{Var}_{X}(s) \operatorname{Var}_{X}(t)\right)^{1 / 2}}$.

Proposición 2.1.10. Sean $E=\mathbb{R} ; \mathfrak{E}=\mathcal{B}_{1} ; X=\left\{X_{s} / s \in S\right.$ y $X_{s}: \Omega \longmapsto$ $E$ es $(\mathcal{F}, \mathfrak{E})$-medible $\}$ un proceso estocástico de imágenes de $2^{\circ}$ orden. Sea $\Lambda \subset S$ con $\#(\Lambda)=n \geq 2$, pongamos $\Lambda:=\left\{s_{1}, \ldots, s_{n}\right\}$. Sea $C_{X, \Lambda}$ la matriz $n \times n$ dada por $C_{X, \Lambda}(i, j)=C_{X}\left(s_{i}, s_{j}\right)$. Entonces $C_{X, \Lambda}$ es simétrica $y$ definida no negativa.

Definición 2.1.11. Sean $E=\mathbb{R} ; \mathfrak{E}=\mathcal{B}_{1} ; X=\left\{X_{s} / s \in S\right.$ y $X_{s}: \Omega \longmapsto$ $E$ es $(\mathcal{F}, \mathfrak{E})$-medible $\}$ un proceso estocástico de imágenes con soporte $S y$ espacio de estados E. Diremos que $X$ es gaussiano si:

Cada vez que $\Lambda:=\left\{s_{1}, \ldots, s_{n}\right\} \subset S$ con $n \geq 1$ y $\left(a_{1}, \ldots, a_{n}\right) \in \mathbb{R}^{n} \backslash\{\tilde{0}\}$ se tiene que $\omega \longmapsto \sum_{i=1}^{n} a_{i} X_{s_{i}}(\omega)$ definida sobre $\Omega$ con valores $\mathbb{R}$ es una v.a. gaussiana.

Notación 2.1.12. De ahora en adelante, salvo expresa mención en contrario, $S$ será un subconjunto de $\mathbb{R}^{2}$ (o de $\mathbb{R}$ según sea el contexto) tal que junto con la operación suma habitual en $\mathbb{R}^{2}$ (o en $\mathbb{R}$ ) forma un grupo.

Definición 2.1.13. Sean $E=\mathbb{R} ; \mathfrak{E}=\mathcal{B}_{1} ; X=\left\{X_{s} / s \in S\right.$ y $X_{s}: \Omega \longmapsto$ $E$ es $(\mathcal{F}, \mathfrak{E})$-medible $\}$ un proceso estocástico de imágenes con soporte $S y$ espacio de estado $E$.

a) Si $X$ es de $1^{\circ}$ orden, diremos que $X$ es débilmente estacionario de $1^{\circ}$ orden si

$$
\exists \mu_{X} \in \mathbb{R} \text { tal que } \mu_{X}(s)=\mu_{X} \forall s \in S .
$$

$Y$ si $\mu_{X}(s)=0 \forall s \in S$, diremos que $X$ es centrado.

b) Si $X$ es de $2^{\circ}$ orden, diremos que $X$ es débilmente estacionario de $2^{\circ}$ orden si

$$
\text { es débilmente estacionario de } 1^{\circ} \text { orden } y
$$

para cada $h \in S, \exists C_{X}^{0}(h) \in \mathbb{R}$ tal que $C_{X}(s+h, s)=C_{X}^{0}(h) \forall s \in S$.

En tal caso, llamaremos función de covarianza de $X$ a $C_{X}^{0}$ en lugar de $C_{X}$.

Pondremos $X$ es $w-\mathcal{L}^{1}$ si $X$ es débilmente estacionario de $1^{\circ}$ orden y $X$ es $w-\mathcal{L}^{2}$ si es débilmente estacionario de $2^{\circ}$ orden. 
Definición 2.1.14. Diremos que $C^{o}: S \longrightarrow \mathbb{R}$ es

- definida no negativa si se cumple que $n \geq 2, \quad\left\{s_{1}, \ldots, s_{n}\right\} \subset S$ y $a_{1}, \ldots, a_{n} \in \mathbb{R} \Rightarrow \sum_{i, j} a_{i} a_{j} C^{o}\left(s_{i}-s_{j}\right) \geq 0$.

- definida positiva si se cumple que

$n \geq 2, \quad\left\{s_{1}, \ldots, s_{n}\right\} \subset S$ y $a_{1}, \ldots, a_{n} \in \mathbb{R}$ no todos nulos $\Rightarrow$

$$
\sum_{i, j} a_{i} a_{j} C^{o}\left(s_{i}-s_{j}\right)>0 \text {. }
$$

Definición 2.1.15. Diremos que $C: S \times S \longrightarrow \mathbb{R}$ es

- definida no negativa (d.n.n.) si se cumple que $n \geq 2, \quad\left\{s_{1}, \ldots, s_{n}\right\} \subset S y a_{1}, \ldots, a_{n} \in \mathbb{R} \Rightarrow \sum_{i, j} a_{i} a_{j} C\left(s_{i}, s_{j}\right) \geq 0$.

- definida positiva (d.p.) si se cumple que

$$
\begin{aligned}
& n \geq 2, \quad\left\{s_{1}, \ldots, s_{n}\right\} \subset S \text { y } a_{1}, \ldots, a_{n} \in \mathbb{R} \text { no todos nulos } \Rightarrow \\
& \sum_{i, j} a_{i} a_{j} C\left(s_{i}, s_{j}\right)>0 .
\end{aligned}
$$

Proposición 2.1.16. Propiedades de la función de covarianza en general. Sean $E=\mathbb{R} ; \mathfrak{E}=\mathcal{B}_{1} ; X=\left\{X_{s} / s \in S\right\}$ con $X_{s} \in \mathcal{L}^{2}(\Omega, \mathcal{F}, P, \mathbb{R}) \forall s \in S$, $C_{X}: S \times S \longrightarrow \mathbb{R}$ la función de covarianza de $X$. Entonces

1. $C_{X}(s, s) \geq 0 \forall s \in S$.

2. $C_{X}\left(s_{1}, s_{2}\right)=C_{X}\left(s_{2}, s_{1}\right) \forall s_{1}, s_{2} \in S$.

3. $C_{X}$ es d.n.n.

4. $\left|C_{X}\left(s_{1}, s_{2}\right)\right|^{2} \leq C_{X}\left(s_{1}, s_{1}\right) C_{X}\left(s_{2}, s_{2}\right) \forall s_{1}, s_{2} \in S$.

5. $\left|C_{X}\left(s_{1}, s\right)-C_{X}\left(s_{2}, s\right)\right|^{2} \leq C_{X}(s, s)\left[C_{X}\left(s_{1}, s_{1}\right)+C_{X}\left(s_{2}, s_{2}\right)\right.$ $\left.-2 C_{X}\left(s_{2}, s_{1}\right)\right] \forall s_{1}, s_{2} \in S$.

Proposición 2.1.17. Propiedades de la función de covarianza en el caso estacionario débil. Sean $E=\mathbb{R} ; \mathfrak{E}=\mathcal{B}_{1}, X=\left\{X_{s} / s \in S\right\} w-\mathcal{L}^{2}, C_{X}^{0}$ : $S \longrightarrow \mathbb{R}$ la función de covarianza de $X$. Entonces

1. $C_{X}^{0}(-h)=C_{X}^{0}(h) \forall h \in S$. 
2. $\left|C_{X}^{0}(h)\right| \leq C_{X}^{0}(0), \forall h \in S$.

3. $C_{X}^{0}$ es d.n.n.

4. $\left|C_{X}^{0}(s+h)-C_{X}^{0}(s)\right|^{2} \leq C_{X}^{0}(0) 2\left[C_{X}^{0}(0)-C_{X}^{0}(h)\right], \forall s$ y $h \in S$.

Proposición 2.1.18. Sean $E=\mathbb{R} ; \mathfrak{E}=\mathcal{B}_{1} ; X_{1}=\left\{X_{1, s} / s \in S\right\}$ y $X_{2}=$ $\left\{X_{2, s} / s \in S\right\}$ con $X_{i, s} \in \mathcal{L}^{2}(\Omega, \mathcal{F}, P, \mathbb{R}) \forall s \in S$ e $i=1,2 ; C_{X_{1}}: S \times S \longrightarrow$ $\mathbb{R}$ y $C_{X_{2}}: S \times S \longrightarrow \mathbb{R}$ la funciones de covarianza de $X_{1}$ y $X_{2}$ respectivamente; $a_{1} \geq 0, a_{2} \geq 0$. Si $X_{1}$ y $X_{2}$ son independientes entonces $X=\left\{X_{s} / s \in S\right\}$ dado por $X_{s}:=\sqrt{a_{1}} X_{1, s}+\sqrt{a_{2}} X_{2, s}$ es un proceso estocástico con $X_{s} \in \mathcal{L}^{2}(\Omega, \mathcal{F}, P, \mathbb{R})$ tal que $C_{X}\left(s_{1}, s_{2}\right)=a_{1} C_{X_{1}}\left(s_{1}, s_{2}\right)+$ $a_{2} C_{X_{2}}\left(s_{1}, s_{2}\right)$.

Proposición 2.1.19. Sean $E=\mathbb{R} ; \mathfrak{E}=\mathcal{B}_{1}, X_{n}=\left\{X_{n, s} / s \in S\right\}$ con $X_{n, s} \in \mathcal{L}^{2}(\Omega, \mathcal{F}, P, \mathbb{R}) \forall s \in S$ y $n=1,2, \ldots$. Supongamos que para cada $s \in S \exists X_{s} \in \mathcal{L}^{2}(\Omega, \mathcal{F}, P, \mathbb{R})$ tal que $X_{n, s} \underset{n \rightarrow \infty}{\longrightarrow} X_{s}$ en $\mathcal{L}^{2}(\Omega, \mathcal{F}, P, \mathbb{R})$ entonces:

a) $X:=\left\{X_{s} / s \in S\right\}$ es un proceso estocástico de imágenes con soporte $S$, espacio de estados $E$ y tal que $X_{s} \in \mathcal{L}^{2}(\Omega, \mathcal{F}, P, \mathbb{R})$.

b) $C_{X}\left(s_{1}, s_{2}\right)=\lim _{n \rightarrow \infty} C_{X_{n}}\left(s_{1}, s_{2}\right) \forall s_{1}, s_{2} \in S$.

\subsection{Funciones de covarianza definidas positi- vas $\mathbf{y}$ procesos gaussianos}

Nota 2.2.1. Sea $X=\left\{X_{s} / s \in S\right\}$ un proceso gaussiano con espacio de estados $E$, tal que $E\left(X_{s}^{2}\right)>0$ y $E\left(X_{s}\right)=0 \forall s \in S$. Entonces $C_{X}$ es una función definida positiva. Recíprocamente, sea $C: S \times S \longrightarrow \mathbb{R}$ una función simétrica definida positiva, entonces existe un proceso gaussiano $X=\left\{X_{s} / s \in S\right\}$ tal que $C_{X}=C$ y $E\left(X_{s}\right)=0 \forall s$. (La recíprocra se prueba recurriendo al teorema de Kolmogorov).

Este resultado permite generalizar en algún sentido los resultados de la Sección anterior.

Proposición 2.2.2. Sea $C: S \times S \longrightarrow \mathbb{R}$ una función definida positiva. Entonces:

1. $C(s, s)>0 \forall s \in S$.

2. $C\left(s_{1}, s_{2}\right)=C\left(s_{2}, s_{1}\right) \forall s_{1}, s_{2} \in S$.

3. $\left|C\left(s_{1}, s_{2}\right)\right|^{2} \leq C\left(s_{1}, s_{1}\right) C\left(s_{2}, s_{2}\right) \forall s_{1}, s_{2} \in S$. 
4. $\left|C\left(s_{1}, s\right)-C\left(s_{2}, s\right)\right|^{2} \leq$ $C(s, s)\left[C\left(s_{1}, s_{1}\right)+C\left(s_{2}, s_{2}\right)-2 C\left(s_{2}, s_{1}\right)\right] \forall s, s_{1}, s_{2} \in S$.

Proposición 2.2.3. Sea $C: S \times S \longrightarrow \mathbb{R}$ definida positiva tal que

$$
C\left(s_{1}, s_{2}\right)=C\left(s_{1}-s_{2}, 0\right) .
$$

Sea $C^{0}(h)=C(h, 0) \forall h \in S$, entonces

1. $C^{0}(-s)=C^{0}(s) \forall s \in S$.

2. $\left|C^{0}(s)\right| \leq C^{0}(0), \forall s \in S$.

3. $C^{0}$ es d.p.

4. $\left|C^{0}(s+h)-C^{0}(s)\right|^{2} \leq C^{0}(0) 2\left[C^{0}(0)-C^{0}(h)\right], \forall s$ y $h \in S$.

En este caso, a veces se dice que $C$ es definida positiva estacionaria, y que $C^{0}$ es definida positiva sobre $S$.

Proposición 2.2.4. Sean $C_{1}: S \times S \longrightarrow \mathbb{R}$ y $C_{2}: S \times S \longrightarrow \mathbb{R}$ definidas positivas; $a_{1} \geq 0, a_{2} \geq 0$ y $a_{1} \neq a_{2}$. Sea $C: S \times S \longrightarrow \mathbb{R}$ definida por

$$
C:=a_{1} C_{1}+a_{2} C_{2}
$$

entonces $C$ es definida positiva.

Proposición 2.2.5. Para cada $n=1,2, \ldots$ sea $C_{n}: S \times S \longrightarrow \mathbb{R}$ definida positiva. Supongamos que para cada $\left(s_{1}, s_{2}\right) \in S \times S$ existe $\lim _{n \rightarrow \infty} C_{n}\left(s_{1}, s_{2}\right)=$ $C\left(s_{1}, s_{2}\right)$, entonces $C$ es definida positiva.

Proposición 2.2.6. Sean $C_{1}: S \times S \longrightarrow \mathbb{R}$ y $C_{2}: S \times S \longrightarrow \mathbb{R}$ definidas positivas. Sea $C: S \times S \longrightarrow \mathbb{R}$ dada por $C\left(s_{1}, s_{2}\right)=C_{1}\left(s_{1}, s_{2}\right) \cdot C_{2}\left(s_{1}, s_{2}\right)$ entonces $C$ es definida positiva.

Proposición 2.2.7. Sean $\mathcal{U} \in \mathcal{B}_{2} ; \mathcal{B}_{\mathcal{U}}:=\mathcal{B}_{2} \cap \mathcal{U} ; \mu$ una medida finita sobre $\left(\mathcal{U}, \mathcal{B}_{\mathcal{U}}\right)$. Sea $\tilde{C}: \mathcal{U} \times S \times S \longrightarrow \mathbb{R}$ tal que

i) Para cada $u \in \mathcal{U}, C_{u}: S \times S \longrightarrow \mathbb{R}$ dada por

$$
C_{u}\left(s_{1}, s_{2}\right):=\tilde{C}\left(u, s_{1}, s_{2}\right)
$$

es definida positiva.

ii) Para cada $\left(s_{1}, s_{2}\right) \in S \times S$ la función $C_{\left(s_{1}, s_{2}\right)}: \mathcal{U} \longrightarrow \mathbb{R}$ dada por

$$
C_{\left(s_{1}, s_{2}\right)}(u)=\tilde{C}\left(u, s_{1}, s_{2}\right)
$$

está en $\mathcal{L}^{2}\left(\mathcal{U}, \mathcal{B}_{\mathcal{U}}, \mu, \mathbb{R}\right)$. 
Sea $C: S \times S \longrightarrow \mathbb{R}$ dada por

$$
C\left(s_{1}, s_{2}\right):=\int_{\mathcal{U}} C_{\left(s_{1}, s_{2}\right)}(u) d \mu(u) .
$$

Entonces $C$ es definida positiva.

Proposición 2.2.8. Sean $C: S \times S \longrightarrow \mathbb{R}$ definida positiva estacionaria. $C^{0}: S \longrightarrow \mathbb{R}$ dada por

$$
C^{0}(s):=C(s, 0) \forall s \in S .
$$

Si $C^{0}$ es continua en 0 , entonces $C^{0}$ es uniformemente continua en todo $S$.

\subsection{Estacionaridad estricta. Isotropía}

Notación 2.3.1. Para cada $k \geq 1$ y cada $\left(s_{1}, \ldots, s_{k}\right) \in S^{k} \operatorname{sea} P_{X,\left(s_{1}, \ldots, s_{k}\right)}$ la probabilidad sobre $\left(E^{k}, \mathfrak{E}^{k}\right)$ dada por

$$
P_{X,\left(s_{1}, \ldots, s_{k}\right)}(B)=P\left(\left(X_{s_{1}}, \ldots, X_{s_{k}}\right) \in B\right), B \in \mathfrak{E}^{k} .
$$

Definición 2.3.2. Se dice que $X$ es estrictamente estacionario si

$$
P_{X,\left(s_{1}, \ldots, s_{k}\right)}=P_{X,\left(s_{1}+h, \ldots, s_{k}+h\right)} \forall\left(s_{1}, \ldots, s_{k}\right) \in S^{k},
$$

con $k \geq 1$ y $\forall h \in S$.

Nota 2.3.3. Supongamos que $E=\mathbb{R} ; \mathfrak{E}=\mathcal{B}_{1} ; X_{s} \in \mathcal{L}^{2}(\Omega, \mathcal{F}, P, \mathbb{R})$ $\forall s \in S$. Si $X$ es estrictamente estacionario, entonces $X$ es débilmente estacionario de $2^{\circ}$ orden. La recíproca no es cierta en general salvo en el caso en que $X$ sea gaussiano.

Definición 2.3.4. Sean $E=\mathbb{R} ; \mathfrak{E}=\mathcal{B}_{1} ; X=\left\{X_{s} / s \in S\right\}$ un proceso estocástico de imágenes con espacio de estados $E$.

a) Si $X$ es i.i.d. centrado y estrictamente estacionario, entonces diremos que $X$ es un ruido blanco en el sentido fuerte (un $\boldsymbol{S} W \mathbf{N}$ ).

b) Si $X$ es de $2^{\circ}$ orden, centrado no correlacionado con $0<E\left(X_{s}^{2}\right)<\infty$ $\forall s \in S$, entonces diremos que $X$ es un ruido blanco en el sentido débil (un $\boldsymbol{W} W N$ ).

c) Si $X$ es de $2^{\circ}$ orden $y \exists C_{X, I}:[0,+\infty) \longrightarrow \mathbb{R}$ tal que

$$
C_{X}\left(s_{1}, s_{2}\right)=C_{X, I}\left(\left\|s_{1}-s_{2}\right\|\right) \forall s_{1}, s_{2} \in S,
$$

entonces diremos que $X$ es isotrópico. $Y$ en este caso llamaremos correlación isotrópica a

$$
\rho_{X, I}(h):=\frac{C_{X, I}(h)}{C_{X, I}(0)} .
$$


Proposición 2.3.5. Sean $E=\mathbb{R} ; \mathfrak{E}=\mathcal{B}_{1} ; X=\left\{X_{s} / s \in S\right\}$ un proceso estocástico de imágenes con espacio de estados $E$.

Si $X$ es centrado e isotrópico, entonces

Proposición 2.3.6. $\quad$ a) Dados $s_{1}, s_{2}$ y $s_{3}$ en $S$ tales que

$$
\left\|s_{1}-s_{2}\right\|=\left\|s_{1}-s_{3}\right\|=h
$$

entonces

$$
3 C_{X, I}(h)\left(1+2 \rho_{X, I}(h)\right)=E\left(\left(\sum_{i=1}^{3} X_{s_{i}}\right)^{2}\right) \geq 0 .
$$

b) $\rho_{X, I}(h) \geq \frac{1}{2} \forall h$.

\subsection{Movimientos Brownianos}

Definición 2.4.1. Sean $E=\mathbb{R}, \mathfrak{E}=\mathcal{B}_{1}, T=[0,+\infty)$. Se dice que $X=$ $\left\{X_{s} / s \in T\right\}$ con espacio de estados $E$ es un movimiento browniano sobre $T$ si:

i) $X_{0} \equiv 0$.

ii) $X_{s} \sim N(0, s) \forall s>0$.

iii) Para cada $0 \leq s<t$ sea

$$
X_{(s, t]}:=X_{t}-X_{s}
$$

Si $k \geq 2 y\left(s_{1}, t_{1}\right] \cap \ldots \cap\left(s_{k}, t_{k}\right]=\phi$ entonces $X_{\left(s_{1}, t_{1}\right]}, \ldots, X_{\left(s_{k}, t_{k}\right]}$ son independientes.

Proposición 2.4.2. Sea $X=\left\{X_{s} / s \in T\right\}$ un movimiento browniano sobre $T=[0,+\infty)$. Entonces

$$
C_{X}(s, t)=\min (\{s, t\}) .
$$

Definición 2.4.3. Sean $E=\mathbb{R} ; \mathfrak{E}=\mathcal{B}_{1} ; T=[0,+\infty) \times[0,+\infty)$. Se dice que $X=\left\{X_{s} / s \in T\right\}$ con espacio de estados $E$ es un movimiento browniano sobre $T$ si:

i) $X_{(u, v)} \equiv 0$ si $u . v=0$ con $(u, v) \in S$.

ii) Si $(u, v) \in T$ y u.v $\neq 0$ entonces $X_{(u, v)} \sim N(0, u . v)$. 
iii) Para cada $\tilde{s}:=\left(s_{1}, s_{2}\right)$ y $\tilde{t}:=\left(t_{1}, t_{2}\right)$ en $T$, pondremos

$$
\begin{aligned}
& \tilde{s} \leq \tilde{t} \text { si } s_{1} \leq t_{1} \text { y } s_{2} \leq t_{2} \\
& \tilde{s}<\tilde{t} \text { si } \tilde{s} \leq \tilde{t} \text { y son diferentes. }
\end{aligned}
$$

Si $\tilde{s} \leq \tilde{t}$, llamaremos rectángulo $\tilde{s}, \tilde{t}$ (en símbolos $(\tilde{s}, \tilde{t}]$ ) a

$$
(\tilde{s}, \tilde{t}]:=\left\{(u, v) / s_{1}<u \leq t_{1}, s_{2}<v \leq t_{2}\right\} .
$$

con $\tilde{s}=\left(s_{1}, s_{2}\right)$ y $\tilde{t}=\left(t_{1}, t_{2}\right)$.

Si $k \geq 2 y\left(\tilde{s}_{1}, \tilde{t}_{1}\right] \cap \ldots \cap\left(\tilde{s}_{k}, \tilde{t}_{k}\right]=\emptyset$ entonces $X_{\left(\tilde{s}_{1}, \tilde{t}_{1}\right]}, \ldots, X_{\left(\tilde{s}_{k}, \tilde{t}_{k}\right]}$ son independientes, donde

$$
X_{(\tilde{s}, \tilde{t}]}:=X_{\left(t_{1}, t_{2}\right)}-X_{\left(t_{1}, s_{2}\right)}-X_{\left(s_{1}, t_{2}\right)}+X_{\left(s_{1}, s_{2}\right)} .
$$

Proposición 2.4.4. Si $X=\left\{X_{\tilde{s}} / \tilde{s} \in[0,+\infty) \times[0,+\infty)\right\}$ es un movimiento browniano sobre $T=[0,+\infty) \times[0,+\infty)$. Entonces $X$ es centrado, gaussiano $y$

$$
C_{X}(\tilde{s}, \tilde{t})=\min \left(\left\{s_{1}, s_{2}\right\}\right) \cdot \min \left(\left\{t_{1}, t_{2}\right\}\right) .
$$

\subsection{Procesos intrínsecos y variogramas}

En toda esta Sección $E=\mathbb{R} ; \mathfrak{E}=\mathcal{B}_{1}$ y $X=\left\{X_{s} / s \in S\right\}$ un proceso de imágenes con soporte $S$ y espacio de estados $E$.

Definición 2.5.1. Se dice que $X$ es intrínsecamente estacionario o simplemente que es intrínseco, si para cada $h \in S$ el proceso

$$
\Delta_{h} X:=\left\{\left(\Delta_{h} X\right)_{s}:=X_{s+h}-X_{s} / s \in S\right\}
$$

es un proceso estacionario de $2^{\circ}$ orden. En este caso definimos la función semivariograma de $X, \gamma_{X}: S \longrightarrow[0,+\infty)$ por

$$
\gamma_{X}(h):=\frac{1}{2} \operatorname{Var}\left(X_{s+h}-X_{s}\right)
$$

cualquiera sea el $s \in S$.

Proposición 2.5.2. Si $X$ es $w-\mathcal{L}^{2}$, entonces $X$ es intrísecamente estacionario $y$

$$
\gamma_{X}(h)=C_{X}(h)-C_{X}(0) \quad \forall h \in S .
$$

Proposición 2.5.3. Sea $X$ de $2^{\circ}$ orden tal que $\exists \alpha \in \mathbb{R}, \beta \in \mathbb{R}$, satisfaciendo

$$
\mu_{X}(s)=\alpha s+\beta \quad \forall s \in S .
$$

$\left\{X_{s}-\mu_{X}(s) / s \in S\right\}$ es $w-\mathcal{L}^{2}$.

Entonces $X$ es intrínseco. 
Definición 2.5.4. Si $X$ es intrínseco definimos función incremento de $X$ a $m_{X}(h)=E\left(X_{s+h}-X_{s}\right)$ cualquiera sea $s \in S$.

Proposición 2.5.5. Sean $S:=\mathbb{R}^{2}, X$ intrínseco. Si $m_{X}$ es continua en $0 \in \mathbb{R}^{2}$, entonces $\exists !\left(a_{1}, a_{2}\right) \in \mathbb{R}^{2}$ tal que:

$$
m_{X}\left(\left(h_{1}, h_{2}\right)\right)=a_{1} h_{1}+a_{2} h_{2} .
$$

Definición 2.5.6. Diremos que un proceso intrínseco X tiene incremento centrado si

$$
m_{X}(h)=0 \forall h \in S .
$$

De ahora en adelante supondremos que $X$ es intrínseco con incremento centrado ( $X$ es intrínseco i.c.).

Definición 2.5.7. Sea $\gamma: S \longrightarrow[0,+\infty)$. Se dice que $\gamma$ es condicionalmente definida negativa (c.d.n.) si se cumple:

$$
\left.\begin{array}{c}
n \geq 2,\left\{s_{1}, \ldots, s_{m}\right\} \subset S \\
a_{1}, \ldots, a_{m} \in \mathbb{R} \operatorname{con} \sum_{i=1}^{m} a_{i}=0
\end{array}\right\} \Rightarrow \sum_{i, j} a_{i} a_{j} \gamma\left(s_{i}-s_{j}\right) \leq 0 .
$$

Proposición 2.5.8. Si $X$ es un proceso intrínseco, entonces

a) $\gamma_{X}(h)=\gamma_{X}(-h) y \gamma_{X}(0)=0$

b) $\gamma_{X}$ es c.d.n.

Proposición 2.5.9. Sea $T: S \longrightarrow S$ tal que:

$$
T\left(s_{1} \pm s_{2}\right)=T\left(s_{1}\right) \pm T\left(s_{2}\right) \forall s_{1}, s_{2} \in S .
$$

Sea $X$ un proceso con soporte S. Sea $X^{T}=\left\{X_{s}^{T} / s \in S\right\}$ dado por $X_{s}^{T}:=X_{T(s)}$. Si $X$ es intrínseco, entonces $X^{T}$ también lo es.

Proposición 2.5.10. Sean $a_{1} \geq 0, a_{2} \geq 0$ reales; $X_{1}=\left\{X_{1, s} / s \in S\right\}$ y $X_{2}=\left\{X_{2, s} / s \in S\right\}$ procesos intrínsecos i.c., independientes. Entonces $X=\left\{X_{s} / s \in S\right\}$ dado por

$$
X_{s}:=\sqrt{a_{1}} X_{1, s}+\sqrt{a_{2}} X_{2, s} \forall s \in S
$$

es un proceso intrínseco i.c.

Definición 2.5.11. Sea $\varphi: \mathbb{R}^{2} \longmapsto \mathbb{R}$. Se dice que $\varphi$ es localmente acotada en s si existe un entorno $V$ de s tal que $\sup \{|\varphi(t)| / t \in V\}<\infty$.

Proposición 2.5.12. Sea $S=\mathbb{R}^{2}$. Sea $X$ un proceso intrínseco i.c. Si $\gamma_{X}$ es continua en $\tilde{0} \in \mathbb{R}^{2}$, entonces $\gamma_{X}$ es continua en si $\gamma_{X}$ es localmente acotada en $s$. 
Proposición 2.5.13. Sean $S=\mathbb{R} ; X$ un proceso i.c. Si $\gamma_{X}$ es localmente acotada en 0 , entonces existen $a \geq 0, b \geq 0$ tales que

$$
\gamma_{X}(s) \leq a\|s\|^{2}+b .
$$

Nota 2.5.14. Notemos que si $X$ es $w-\mathcal{L}^{2}$, entonces $C_{X}$ es acotada. No es asi en el caso de $\gamma_{X}$. En efecto si $X$ es un movimiento browniano sobre $S=[0,+\infty)$ entonces:

$$
\gamma_{X}(h)=\frac{1}{2} h \quad \forall h \geq 0 .
$$

Nota 2.5.15. Por la Proposición 2.5.13 sabemos que si $X$ es intrínseco i.c. y si $\gamma_{X}$ es localmente acotada en 0 , entonces $\exists a \geq 0$ tal que

$$
\frac{\gamma_{X}(s)}{\|s\|^{2}} \leq a+\frac{b}{\|s\|^{2}} \quad \forall s \in \mathbb{R} .
$$

Veamos un caso en el que vale la igualdad.

Sean $Z_{0}$ y $Z_{1}$ v.a. tales que:

- $E\left(Z_{0}\right)=E\left(Z_{1}\right)=0$ y $\operatorname{Var}\left(Z_{0}\right)<\infty, \operatorname{Var}\left(Z_{1}\right)<\infty$.

- $Z_{0}$ y $Z_{1}$ son independientes.

Sea $\operatorname{Var}\left(Z_{1}\right)=\sigma_{1}^{2} S=\mathbb{R}$. Para cada $t \in \mathbb{R}$ sea

$$
X_{t}=Z_{0}+t Z_{1}
$$

Entonces $X=\left\{X_{t} / t \in S\right\}$ es intrínseco i.c. $y \gamma_{X}(t)=\frac{1}{2} \sigma_{1}^{2} t^{2} \forall t \in \mathbb{R}$.

\subsection{Variogramas para procesos estacionarios}

En esta Sección supondremos que: $S=\mathbb{R}$ o $S=\mathbb{R}^{2} ; X=\left\{X_{s} / s \in S\right\}$ es un proceso $w-\mathcal{L}^{2}$. Por la Proposición 2.5.2 tenemos que $X$ es intrínsecamente estacionario y $\gamma_{X}(h)=C_{X}(h)-C_{X}(0) \quad \forall h \in S$.

Proposición 2.6.1. Si $C_{X}(h) \underset{\|h\| \rightarrow \infty}{\longrightarrow} 0$ entonces $\gamma_{X}(h) \underset{\|h\| \rightarrow \infty}{\longrightarrow} C_{X}(0)$. En este caso, al valor $C_{x}(0)$ lo llamaremos meseta en $\|h\| \rightarrow \infty$.

Definición 2.6.2. Supongamos que $\gamma_{X}$ es no decreciente. Definimos:

a) Rango del semivariograma de $X$ a

$$
r_{\gamma_{X}}:=\min \left\{\|h\| / \gamma_{X}(h)=C_{X}(0)\right\} .
$$

b) Rango práctico del semivariograma de $X$ a

$$
r p_{\gamma_{X}}:=\min \left\{\|h\| / \gamma_{X}(h)=0.95 C_{X}(0)\right\} .
$$




\subsection{Ejemplos de covarianzas y variogramas}

Semivariogramas isotrópicos

Definición 2.7.1. Se dice que $X$ tiene un semivariograma

a) efecto pepita puro si: $\exists \sigma^{2}>0$ tal que

$$
\gamma_{X}(h)= \begin{cases}\sigma^{2} & \text { si } h \neq 0 \\ 0 & \text { si } h=0\end{cases}
$$

b) exponencial con parámetro $a>0$ y $\sigma^{2}>0$ si:

$$
\gamma_{X}(h)=\sigma^{2}\left(1-\exp \left(-\frac{\|h\|}{a}\right)\right) \text {. }
$$

c) esférico con parámetros $a>0 \quad y \sigma^{2}>0$ si:

$$
\gamma_{X}(h)=\left\{\begin{array}{l}
\sigma^{2}\left(\frac{3}{2} \frac{\|h\|}{a}-\frac{1}{2}\left(\frac{\|h\|}{a}\right)^{3}\right) \quad \text { si }\|h\| \leq a \\
\sigma^{2} \text { si }\|h\|>a .
\end{array}\right.
$$

d) exponencial generalizado con parámetros $a>0, \sigma^{2}>0$ y $0<$ $\alpha \leq 2$ si:

$$
\gamma_{X}(h)=\sigma^{2}\left(1-\exp \left(-\frac{\|h\|}{a}\right)^{\alpha}\right) .
$$

Si $\alpha=2$ se llamará gaussiano con parámetros $a>0 \quad \boldsymbol{y} \sigma^{2}>0$.

e) Matérn

Para la definición de este tipo de semivariograma, necesitamos una definición previa:

Definición 2.7.2. Sea $v>-1$ real. Se llama función de Bessel modificada de $2^{o}$ clase con parámetro $v$ a $K_{v}:[0,+\infty) \rightarrow \mathbb{R}$ dada por:

$$
K_{v}(z):=\frac{\Gamma\left(v+\frac{1}{2}\right)(2 z)^{v}}{\sqrt{\pi}} \int_{0}^{+\infty} \frac{\cos (t)}{\left(t^{2}+z^{2}\right)^{v+\frac{1}{2}}} d t ; z \geq 0 .
$$

Propiedades y más detalles de esta función se pueden ver en:

http://www.mathworld.wolram.com.

Se dice que $X$ tiene un semivariograma Matérn con parámetros $v>-1, a>0$ y $\sigma^{2}>0$ si

$$
\gamma_{X}(h)=\sigma^{2}\left(1-\frac{2^{1-v}}{\Gamma(v)}\left(\frac{\|h\|}{a}\right)^{v} K_{v}\left(\frac{\|h\|}{a}\right)\right) .
$$


f) potencial con parámetro $b>0$ y $0<c \leq 2$ si

$$
\gamma_{X}(h)=b\|h\|^{c} .
$$

Proposición 2.7.3. Sean: $k \geq 2$ entero; para cada $i=1, \ldots, k$ sea $X_{i}=$ $\left\{X_{i, s} / s \in S\right\}$ un proceso de imágenes con soporte $S$ y espacio de estados $E=\mathbb{R}$. Supongamos que sea centrado y que $E\left(X_{i, s} X_{j, t}\right)=0$ si $i \neq j y$ $\forall s, t \in S$

a) Si $X_{i}$ es intrínseco para todo $i=1, \ldots, k$ entonces $X=\sum_{i=1}^{k} X_{i}$, $\left(X_{s}:=\sum_{i=1}^{k} X_{i, s}, \forall s \in S\right)$ es intrínseco $y$

$$
\gamma_{X}(h)=\sum_{i=1}^{k} \gamma_{X_{i}}(h) \quad \forall h .
$$

b) Si $X_{i}$ es $w-\mathcal{L}^{2}$ para todo $i=1, \ldots, k$ entonces $X$ es $w-\mathcal{L}^{2} y$

$$
C_{X}(s)=\sum_{i=1}^{k} C_{X_{i}}(s) \quad \forall s .
$$

\subsection{Anisotropía}

En esta Sección supondremos $S=\mathbb{R}^{2}$ y que $X=\left\{X_{s} / s \in S\right\}$ es un proceso intrínseco.

Definición 2.8.1. Sea $\tilde{e} \in \mathbb{R}^{2}$ con $\|\tilde{e}\|=1$. Llamamos semivariograma de $X$ en la dirección de ẽ a $\gamma_{X, \tilde{e}}: \mathbb{R} \longmapsto[0,+\infty)$ dada por

$$
\gamma_{X, \tilde{e}}(h)=\gamma_{X}(h \tilde{e})=\frac{1}{2} E\left(\left(X_{s+h \tilde{e}}-X_{s}\right)^{2}\right) \quad \forall s .
$$

Definición 2.8.2. Diremos que $X$ es anisotrópico o más precisamente que $X$ tiene un semivariograma anisotrópico si existen $\tilde{e}_{1} y \tilde{e}_{2}$ distintos de norma 1 tales que $\gamma_{X, \tilde{e}_{1}} \neq \gamma_{X, \tilde{e}_{2}}$.

Definición 2.8.3. Diremos que $X$ es isotrópico o más precisamente que $X$ tiene un semivariograma isotrópico si dados $h_{1}$ y $h_{2} \in \mathbb{R}^{2}$ con $\left\|h_{1}\right\|=\left\|h_{2}\right\| \Rightarrow \gamma_{X}\left(h_{1}\right)=\gamma_{X}\left(h_{2}\right)$.

Nota 2.8.4. $X$ es isotrópico si y sólo si $X$ no es anisotrópico.

Definición 2.8.5. Se dice que un proceso anisotrópico $X$ tiene anisotropía geométrica si $\exists T: \mathbb{R}^{2} \longmapsto \mathbb{R}^{2}$ lineal y biyectiva tal que $X^{T}=$ $\left\{X_{T(s)} / s \in \mathbb{R}^{2}\right\}$ es isotrópico. 


\subsection{Propiedades Geométricas: continuidad y diferenciabilidad}

En esta Sección supondremos $S=\mathbb{R}$ o $\mathbb{R}^{2}, X$ un proceso de $2^{\circ}$ orden.

Definición 2.9.1. Diremos que $X$ es continuo en media cuadrática en $s \in S$ (c.m.c. en $s \in S$ ) si:

$$
\left(s_{n}\right)_{n \geq 1} \text { en } S, s_{n} \underset{n \rightarrow \infty}{\longrightarrow} s \in S \Rightarrow X_{s_{n}} \stackrel{\mathcal{L}^{2}}{\longrightarrow} X_{s} .
$$

Proposición 2.9.2. Supongamos que $X$ es centrado. Entonces:

$X$ es c.m.c. en $s, \forall s \in S \Longleftrightarrow C_{X}$ es continua en $(s, s) \forall s \in S$.

Definición 2.9.3. Sea $\omega \in \Omega$

a) Llamaremos trayectoria de $X$ en $\omega$ a la función $\operatorname{tr} X_{\omega}: S \longmapsto \mathbb{R}$ dada por $\operatorname{tr} X_{\omega}(s)=X_{s}(\omega) \forall s \in S$.

b) Diremos que $X$ tiene casi seguramente (c.s.) trayectorias continuas si

$\exists \Omega_{0} \subset \Omega$ con $P\left(\Omega_{0}\right)=1$ tal que si $\omega \in \Omega_{0} \Rightarrow \operatorname{tr} X_{\omega}$ es continua en $s$, $\forall s \in S$.

Teorema 2.9.4. Supongamos que:

i) $X$ es gaussiano centrado.

ii) $C_{X}$ es continua.

iii) Existen $0<c<\infty$ y $\varepsilon>0$ tales que: $s \in S, t \in S, E\left(\left(X_{s}-X_{t}\right)^{2}\right) \leq$ $c|\ln (\| s-t||)|^{-(1+\varepsilon)}$.

Entonces $X$ tiene c.s. trayectorias continuas.

Demostración. Ver Adler (1981) ([1]).

Corolario 2.9.5. Si $X$ es gaussiano intrínseco y centrado y existen $0<$ $c<\infty$ y $\varepsilon>0$ tales que: $\gamma_{X}(h) \leq c|\ln (\|h\|)|^{-(1+\varepsilon)} \forall h \in S$. Entonces $X$ tiene c.s. trayectorias continuas.

Ejercicio 2.9.6. Sea $X$ un proceso gaussiano centrado e intrínseco. Supongamos que $\gamma_{X}$ satisface una cualquiera de las definiciones a) - f) de la Definición 2.7.1. Entonces $X$ tiene c.s. trayectorias continuas.

Definición 2.9.7. Sea $S \subset \mathbb{R}$ abierto. Diremos que $X$ es diferenciable en media cuadrática en $s \in S$ ( $X$ es d.m.c. en $s \in S)$ si $\exists \dot{X}_{s} \in$ $\mathcal{L}^{2}(\Omega, \mathcal{F}, P, \mathbb{R})$ tal que

$$
\frac{1}{h}\left(X_{s+h}-X_{s}\right) \underset{|h| \downarrow 0}{\stackrel{\mathcal{L}^{2}}{\longrightarrow}} \dot{X}_{s}
$$


Proposición 2.9.8. Sea $S \subset \mathbb{R}$ abierto. Si $X$ es d.m.c. en s, entonces $X$ es c.m.c. en s.

Ejercicio 2.9.9. Sean: $U$ y $V$ v.a. sobre $(\Omega, \mathcal{F}, P)$ tales que: $U \sim$ $\mathcal{U}(0,2 \pi), V \sim$ Cauchy patrón (esto es, $V$ tiene una densidad $f_{V}$ dada por $\left.f_{V}(x)=\frac{1}{\pi\left(1+x^{2}\right)} \forall x \in \mathbb{R}\right)$ y $U$ y $V$ son independientes. Sea $S:=\mathbb{R}$. Para cada $s \in S$ sea $X_{s}:=\cos (U+s V)$. Entonces $X$ es un proceso de $2^{\circ}$ orden tal que:

a) $E\left(X_{s}\right)=0 \forall s$.

b) $E\left(X_{s} X_{t}\right)=\frac{1}{2} e^{-|s-t|} \forall s, t \in S$.

c) $\operatorname{tr} X_{\omega}$ es infinitamente diferenciable en $s, \forall s \in S$.

d) $X$ no es d.m.c. en $s, \forall s \in S$.

Notación 2.9.10. Con $D_{1,2}$ denotaremos al operador derivada parcial con respecto a la $2^{\circ}$ componente y luego con respecto a la $1^{\circ}$.

Proposición 2.9.11. Sea $X$ en $\mathcal{L}^{2}$ centrado (no necesariamente débilmente estacionario). Supongamos que $\forall s \in \mathbb{R} \exists\left(D_{1,2} C\right)(s, s)$ y $\left|\left(D_{1,2} C\right)(s, s)\right|<$ $\infty \forall s \in \mathbb{R}$. Entonces:

a) $X$ es d.m.c. en $s \in \mathbb{R} y \forall s \in \mathbb{R}$ sea $\dot{X}_{s}$ la derivada.

b) $\exists\left(D_{1,2} C\right)(s, t) \forall(s, t) \in \mathbb{R}^{2}$.

c) $\left(D_{1,2} C\right)(s, t)=C_{\dot{X}}(s, t)$.

\subsection{Continuidad y diferenciabilidad en el caso estacionario}

En esta Sección supondremos: $S=\mathbb{R} ; E=\mathbb{R} ; X$ es un proceso intrínseco.

Proposición 2.10.1. Supongamos que $\exists \gamma_{X}^{\prime \prime}(0)$. Entonces

a) $\exists \gamma_{X}^{\prime \prime}(h), \forall h \in \mathbb{R}$.

b) $X$ es d.m.c. $\forall s \in \mathbb{R}$.

c) $\dot{X}:=\left\{\dot{X}_{s} / s \in \mathbb{R}\right\}$ es $w-\mathcal{L}^{2}$.

d) $\gamma_{X}^{\prime \prime}(h)=C_{\dot{X}}(s+h, s) \forall s, h \in \mathbb{R}$.

e) $\gamma_{X}^{\prime}(t)=E\left(\dot{X}_{s+t} X_{s}\right)$ con $s, t \in \mathbb{R} \quad y \quad-\gamma_{X}^{\prime}(t)=E\left(X_{s+t} \dot{X}_{s}\right)$ con $s, t \in \mathbb{R}$. 
Corolario 2.10.2. Supongamos que $\exists \gamma_{X}^{\prime \prime}(0)$. Como $\gamma_{X}^{\prime}(0)=0$ (pues 0 es un punto mínimo de $\left.\gamma_{X}\right)$ se tiene que $E\left(\dot{X}_{s} X_{s}\right)=0 \forall s \in \mathbb{R}$, esto es, $\dot{X}_{s}$ $y X_{s}$ son no correlacionados $\forall s \in \mathbb{R}$.

Lema 2.10.3. Sean $\left(Y_{n}\right)_{n \geq 1}$ y $Z$ en $\mathcal{L}^{2}(\Omega, \mathcal{F}, P, \mathbb{R})$ con $\mu_{n}=E\left(Y_{n}\right)$, $\sigma_{n}^{2}=E\left(\left(Y_{n}-\mu_{n}\right)^{2}\right), \mu=E(Z)$ y $\sigma^{2}=E\left((Z-\mu)^{2}\right)$. Si $Y_{n} \underset{n \rightarrow \infty}{\longrightarrow} Z$ en $\mathcal{L}^{2}$ entonces:
a) $\mu_{n} \underset{n \rightarrow \infty}{\longrightarrow} \mu$.
b) $\sigma_{n}^{2} \underset{n \rightarrow \infty}{\longrightarrow} \sigma^{2}$.
c) $Y_{n} \underset{n \rightarrow \infty}{\stackrel{\mathcal{D}}{\longrightarrow}} Z$ (convergencia en distribución).
d) Si además $Y_{n} \sim \mathcal{N}\left(\mu_{n}, \sigma_{n}\right)$ y $\sigma>0$ entonces $Z \sim \mathcal{N}(\mu, \sigma)$.

Corolario 2.10.4. Si $\exists \gamma_{X}^{\prime \prime}(0)$ y $X$ es gaussiano, entonces:

a) $\dot{X}$ es gaussiano.

b) $X_{s}$ y $\dot{X}_{s}$ son independientes, $\forall s \in S$.

Corolario 2.10.5. Supongamos que $X$ es estacionario (más precisamente $\left.w-\mathcal{L}^{2}\right)$ y que $C_{X}^{0}$ es dos veces diferenciable. Entonces:

a) $\left(C_{X}^{0}\right)^{\prime \prime}(s-t)=-\left(D_{12} C_{X}\right)(s, t)$ con $s, t \in \mathbb{R}$.

b) $X$ es d.m.c., $\dot{X}$ es $w-\mathcal{L}^{2} y$

$$
C_{\dot{X}}^{0}(h)=-\left(C_{X}^{0}\right)^{\prime \prime}(h) \forall h \in \mathbb{R} .
$$

Notación 2.10.6. Si $X$ es d.m.c., diremos que $X$ es d.m.c. de orden 1. En tal caso pondremos: $X^{(1)}:=\dot{X}$.

Definición 2.10.7. Diremos que $X$ es d.m.c. de orden $m \geq 2$ si $X^{(m-1)}$ es d.m.c. de orden $m-1$ y pondremos $X^{(m)}:=\left(X^{(m-1)}\right)^{-}$.

Corolario 2.10.8. Supongamos:

i) $X$ es $w-\mathcal{L}^{2}$.

ii) Sea $m \geq 1$ entero, $C_{X}^{0}$ es $2 m$-veces diferenciable en todo $t \in \mathbb{R} y$

$\left|\left(C_{X}^{0}\right)^{(2 m)}(0)\right|<\infty$.
Entonces

a) $X$ es d.m.c. de orden $m$. 
b) $C_{X^{(m)}}^{0}(t)=(-1)^{m}\left(C_{X}^{0}\right)^{(2 m)}(t), t \in \mathbb{R}$.

Definición 2.10.9. Diremos que $X$ es d.m.c. infinitamente (o de orden infinito) si es d.m.c. de orden $m \forall m \geq 1$.

Proposición 2.10.10. Supongamos que para todo $m \geq 1$ entero se tiene que $\left|\gamma_{X}^{(m)}(0)\right|<\infty$. Entonces:

a) $X$ es d.m.c. de orden infinito.

b) $\forall t \in \mathbb{R} \frac{1}{k !} \sum_{k=0}^{n} t^{k} X_{0}^{(k)} \underset{\mathcal{L}^{2}}{\longrightarrow} X_{t}$.

\subsection{Representación espectral de covarian-} zas

\subsubsection{Caso $S=\mathbb{R}^{2}$}

Nota 2.11.1. Se puede ver, por ejemplo en: Schlather (1999) ([11]) que:

a) $C^{0}: \mathbb{R}^{2} \longmapsto \mathbb{R}$ satisface que $C^{0}(-X)=C^{0}(X)$ es definida positiva, continua $y$ acotada si y sólo si existe una medida (no negativa) $F_{C^{0}}$ tal que: es simétrica con respecto a $(0,0)$ y

$$
C^{0}(\tilde{h})=\int_{\mathbb{R}^{2}} \cos (<\tilde{h}, \tilde{t}>) F_{C^{0}}(d \tilde{t}),
$$

donde $<\tilde{h}, \tilde{t}>:=h_{1} t_{1}+h_{2} t_{2}$ si $\tilde{h}=\left(h_{1}, h_{2}\right)$ y $\tilde{t}=\left(t_{1}, t_{2}\right)$.

b) $S i C^{0} \in \mathcal{L}^{1}\left(\mathbb{R}^{2}, \mathcal{B}_{2}, \mathbb{R}\right)$ entonces $F_{0}$ es diferenciable p.p. sobre $\mathbb{R}^{2}$ con derivada $f_{C^{0}}$ que llamaremos derivada espectral de $C^{0}$. Esta $f_{C^{0}}$ esta dada por:

$$
f_{C^{0}}(\tilde{t})=\left(\frac{1}{2 \pi}\right)^{2} \int_{\mathbb{R}^{2}} \cos (<\tilde{h}, \tilde{t}>) C^{0}(\tilde{h}) d \tilde{h} .
$$

c) Sea $C^{0}: \mathbb{R}^{2} \longmapsto \mathbb{R}$ definida positiva e isotrópica (esto es $\exists C_{I}^{0}:[0,+\infty$ )

$\longmapsto \mathbb{R}$ tal que $\left.C^{0}(\tilde{h})=C_{I}^{0}(\|\tilde{h}\|)\right)$. Entonces

$$
C_{I}^{0}(\|\tilde{h}\|)=2 \pi \int_{[0,+\infty)} x J_{0}(x\|\tilde{h}\|) f_{2}(x) d x
$$

donde $\forall x \geq 0$

$$
J_{0}(x):=\frac{1}{2 \pi} \int_{0}^{+\infty} \sin (x \cdot \cosh (t)) d t
$$

y $f_{2}:[0,+\infty) \longmapsto \mathbb{R}$ tal que $f_{2}(x):=f_{C^{0}}(\tilde{u})$ con $x=\|\tilde{u}\|$. 


\subsubsection{Caso $S=\mathbb{R}$}

Similar al caso anterior cambiando solamente las expresiones de $C^{0}$ y $f_{C^{0}}$.

a) $C^{0}: \mathbb{R} \longmapsto \mathbb{R}$ está dada por:

$$
C^{0}(h)=\int_{\mathbb{R}} \cos (h t) F_{C^{0}}(d t)
$$

con $F_{C^{0}}$ medida finita (no negativa) sobre $\left(\mathbb{R}, \mathcal{B}_{1}\right)$.

b) $\mathrm{Si} C^{0} \in \mathcal{L}^{1}\left(\mathbb{R}, \mathcal{B}_{1}, \mathbb{R}\right)$, entonces $F_{C^{0}}$ es diferenciable p.p. sobre $\mathbb{R}$ con derivada $f_{C^{0}}$ que llamaremos derivada espectral de $C^{0}$. Esta $f_{C^{0}}$ está dada por:

$$
f_{C^{0}}(t)=\frac{1}{2 \pi} \int_{\mathbb{R}} \cos (h t) C^{0}(h) d h .
$$

c) Sea $C^{0}: \mathbb{R} \longmapsto \mathbb{R}$ definida positiva y par. Entonces

$$
C^{0}(|h|)=2 \int_{0}^{+\infty} \cos (x|h|) f_{1}(x) d x
$$

donde $f_{1}(x):=f_{C^{0}}(u)$ y $x=|u| \forall u \in \mathbb{R}$.

\subsubsection{Caso $S=\mathbb{Z}^{2}$}

a) $C^{0}: S \longmapsto \mathbb{R}$ satisface:

i) $C^{0}(-\tilde{x})=C^{0}(\tilde{x}) \forall \tilde{x} \in S$,

ii) $C^{0}$ es acotada.

iii) $C^{0}$ es definida no negativa.

si y sólo si existe $F_{0}$ medida finita sobre $([0,2 \pi) \times[0,2 \pi)$,

$\left.\mathcal{B}_{2} \cap[0,2 \pi) \times[0,2 \pi)\right)$ tal que:

$$
C^{0}(\tilde{h})=\int \cos (\langle\tilde{h}, \tilde{t}\rangle) F_{C^{0}}(d t) .
$$

b) Si $C^{0}$ es cuadrado sumable $\left(\sum_{\tilde{h} \in S}\left(C^{0}(\tilde{h})\right)^{2}<\infty\right)$, entonces $F_{C^{0}}$ es diferenciable p.p. con derivada dada por:

$$
f_{C^{0}}(\tilde{t})=\left(\frac{1}{2 \pi}\right)^{2} \sum_{\tilde{h} \in \mathbb{Z}^{2}} \cos (<\tilde{h}, \tilde{t}>) C^{0}(\tilde{h}) .
$$

c) En la situación del inciso anterior, si $\sum_{\tilde{h} \in S}\left|C^{0}(\tilde{h})\right|<\infty$, entonces $f_{C^{0}}$ es continua. 


\subsection{Modelos Autorregresivos Espaciales}

Definición 2.12.1. Sea $E=\mathbb{R} ; \mathfrak{E}=\mathcal{B}_{1} ; S=\mathbb{Z}^{2}$;

$$
\begin{aligned}
\tilde{c} & :=\left\{c_{\tilde{s}} / \tilde{s} \in \mathbb{Z}^{2}\right\} \text { tal que } \sum_{\tilde{s} \in \mathbb{Z}^{2}} c_{\tilde{s}}^{2}<\infty ; \\
\eta & :=\left\{\eta_{\tilde{s}} / \tilde{s} \in \mathbb{Z}^{2}\right\} \text { un proceso } S W N .
\end{aligned}
$$

Se dice que $X:=\left\{X_{\tilde{s}} / \tilde{s} \in \mathbb{Z}^{2}\right\}$ es un proceso de medias móviles de orden infinito con proceso de innovaciones $\eta y$ coeficientes $\tilde{c}$ si:

$$
X_{\tilde{s}}=\sum_{\tilde{t} \in \mathbb{Z}^{2}} c_{\tilde{t}} \eta_{\tilde{s}-\tilde{t}} \text { en } \mathcal{L}^{2}(\Omega, \mathcal{F}, P, \mathbb{R}) .
$$

Proposición 2.12.2. Sea la situación de la definición anterior. Entonces:

a) $X$ es $W-\mathcal{L}^{2}$;

b) $C_{X}^{0}(\tilde{u})=\frac{\sigma_{\eta}^{2}}{(2 \pi)^{2}} \sum_{\tilde{h} \in \mathbb{Z}^{2}} \sum_{\tilde{t} \in \mathbb{Z}^{2}} c_{\tilde{t}} c_{\tilde{t}+\tilde{h}} \cos (\langle\tilde{u}, \tilde{t}\rangle) \forall \tilde{u} \in \mathbb{Z}^{2}$.

c) La densidad espectral de $C_{X}^{0}$ es: para todo $: \widetilde{\lambda} \in \mathbb{T}^{2}:=[-\pi, \pi) \times[-\pi, \pi)$

$$
f_{C_{X}^{0}}(\widetilde{\lambda})=\frac{\sigma_{\eta}^{2}}{(2 \pi)^{2}}\left|\sum_{\tilde{t} \in \mathbb{Z}^{2}} c_{\tilde{t}} \cos (\langle\tilde{\lambda}, \tilde{t}\rangle)\right|^{2}
$$

donde $\sigma_{\eta}^{2}:=E\left(\eta_{\tilde{s}}^{2}\right)$, para todo $\tilde{s} \in \mathbb{Z}^{2}$

Definición 2.12.3. Consideremos la situación de la definición anterior. Se dice que $X$ es un proceso de medias móviles de orden finito con proceso de innovaciones $\eta$ y coeficientes $\tilde{c}$ si

$$
\#\left\{\tilde{s} / c_{\tilde{s}} \neq 0\right\}<\infty .
$$

\subsubsection{Modelos ARMA}

Notación 2.12.4. Sea $\tilde{s} \in \mathbb{Z}^{2}$. Con $B^{\tilde{s}}$ denotamos a la funciones definidas sobre $\mathbb{R}^{\mathbb{Z}^{2}}$ en $\mathbb{R}^{\mathbb{Z}^{2}}$ dada por:

$$
B^{\tilde{s}}(\tilde{x})(\tilde{t}):=\tilde{x}(\tilde{t}-\tilde{s}) \quad \forall \tilde{t} \in \mathbb{Z}^{2}, \forall \tilde{x} \in \mathbb{R}^{\mathbb{Z}^{2}} .
$$

Pondremos $\mathbb{Z}_{+}^{2}:=\left\{(i, j) \in \mathbb{Z}^{2} / i \geq 0, j \geq 0\right\}$.

Definición 2.12.5. Sean $R \subset \mathbb{Z}^{2}, M \subset \mathbb{Z}^{2}$ finitos; $\left\{\phi_{\tilde{r}} / \tilde{r} \in R\right\} \subset \mathbb{R}$; $\left\{\theta_{\tilde{m}} / \tilde{m} \in M\right\} \subset \mathbb{R}$ e $I: \mathbb{R}^{\mathbb{Z}^{2}} \longrightarrow \mathbb{R}^{\mathbb{Z}^{2}}$ la identidad; 


$$
\begin{aligned}
P(B): \mathbb{R}^{\mathbb{Z}^{2}} \longrightarrow \mathbb{R}^{\mathbb{Z}^{2}} y Q(B) & : \mathbb{R}^{\mathbb{Z}^{2}} \longrightarrow \mathbb{R}^{\mathbb{Z}^{2}} \text { dadas por: } \\
P(B) & :=\sum_{\tilde{r} \in R} \phi_{\tilde{r}} B^{\tilde{r}} \\
Q(B) & :=\sum_{\tilde{m} \in M} \theta_{\tilde{m}} B^{\tilde{m}} .
\end{aligned}
$$

$\eta=\left\{\eta_{\tilde{s}} / \tilde{s} \in \mathbb{Z}^{2}\right\}$ un proceso $S W N$.

Si $X=\left\{X_{\tilde{s}} / \tilde{s} \in \mathbb{Z}^{2}\right\}$ satisface:

1. $X_{\tilde{s}} \in \mathcal{L}^{2}(\Omega, \mathcal{F}, P, \mathbb{R}) \forall \tilde{s} \in \mathbb{Z}^{2}$.

2. $P(B)(X)(\tilde{s})=Q(B)(\eta)(\tilde{s}) \forall \tilde{s} \in \mathbb{Z}^{2}$

$\left(\Leftrightarrow X_{\tilde{s}}+\sum_{\tilde{r} \in R} \phi_{\tilde{r}} X_{\tilde{s}-\tilde{r}}=\eta_{\tilde{s}}+\sum_{\tilde{m} \in M} \theta_{\tilde{m}} \eta_{\tilde{s}-\tilde{m}}\right)$.

Diremos que $X$ es un $A R M A(P, Q)$ con proceso de innovaciones $\eta$.

Proposición 2.12.6. Consideremos la situación de la definición anterior. Supongamos que

$$
\begin{aligned}
& P\left(z_{1}, z_{2}\right) \neq 0 \quad \forall\left(z_{1}, z_{2}\right) \in \Pi^{2} \text { donde } \\
& P\left(z_{1}, z_{2}\right)=1-\sum_{\left(r_{1}, r_{2}\right) \in R} \phi_{\left(r_{1}, r_{2}\right)} z_{1}^{r_{1}} z_{2}^{r_{2}} y \\
& \Pi^{2}:=\left\{\left(z_{1}, z_{2}\right) \in \mathbb{C}^{2} /\left|z_{1}\right|=\left|z_{2}\right|=1\right\} .
\end{aligned}
$$

Entonces $\exists X$ que es un $\operatorname{ARMA}(P, Q) w-\mathcal{L}^{2}$, con proceso de innovación $\eta=\left\{\eta_{\tilde{s}} / \tilde{s} \in \mathbb{Z}^{2}\right\}$ y además

$$
f_{c_{X}^{0}}(\tilde{t})=\frac{\sigma_{\eta}^{2}}{(2 \pi)^{2}}\left|\frac{Q\left(e^{i t_{1}}, e^{i t_{2}}\right)}{P\left(e^{i t_{1}}, e^{i t_{2}}\right)}\right|^{2}, \quad \forall \tilde{t}=\left(t_{1}, t_{2}\right) \in[0,2 \pi) \times[0,2 \pi)
$$

con $\sigma_{\eta}^{2}:=E\left(\eta_{\tilde{s}}^{2}\right)$, para todo $\tilde{s} \in \mathbb{Z}^{2}$.

\subsection{Procesos $S A R$ (Simultáneos AR)}

Definición 2.13.1. Sean $R \subset \mathbb{Z}^{2}$ finito tal que $(0,0) \notin R ; A_{R}:=\left\{a_{\tilde{r}} / \tilde{r} \in R\right\} \subset$ $\mathbb{R} ; \eta:=\left\{\eta_{\tilde{s}} / \tilde{s} \in \mathbb{Z}^{2}\right\}$ un proceso $S W N$.

Si $X=\left\{X_{\tilde{s}} / \tilde{s} \in \mathbb{Z}^{2}\right\}$ satisface:

a) $E\left(X_{\tilde{s}}\right)=0 \forall \tilde{s} \in \mathbb{Z}^{2}$.

b) $X_{\tilde{s}} \in \mathcal{L}^{2}(\Omega, \mathcal{F}, P, \mathbb{R}) \forall \tilde{s} \in \mathbb{Z}^{2}$.

c) $X_{\tilde{s}}=\sum_{\tilde{r} \in R} a_{\tilde{r}} X_{\tilde{s}-\tilde{r}}+\eta_{\tilde{s}}$ en $\mathcal{L}^{2}(\Omega, \mathcal{F}, P, \mathbb{R})$. 
d) $E\left(X_{\tilde{s}} \eta_{\tilde{t}}\right)=0$ si $\tilde{s} \neq \tilde{t}$.

Entonces diremos que $X$ es un proceso $S A R$ con coeficientes $A_{R}$ y proceso de innovación $\eta$.

Proposición 2.13.2. En la situación de la definición anterior, si

$$
P\left(e^{i \tilde{\lambda}}\right):=1-\sum_{\tilde{s} \in R} a_{\tilde{s}} \exp (i\langle\tilde{\lambda}, \tilde{s}\rangle) \neq 0 \quad \forall \tilde{\lambda} \in \Pi^{2}
$$

entonces

1. Existe $X$, proceso $S A R$ con coeficientes $A_{R}$ y proceso de innovación $\eta$.

2. $C_{X}^{0}$ tiene densidad espectral dada por

$$
f_{C_{X}^{0}}(\tilde{\lambda})=\frac{\sigma_{\eta}^{2}}{(2 \pi)^{2}}\left|\frac{1}{P\left(e^{i \tilde{\lambda}}\right)}\right|^{2}, \quad \tilde{\lambda} \in \Pi^{2} .
$$

Demostración. Se puede ver en Guyon (1995) ([7]).

Ejemplo 2.13.3. SAR isotrópico para entorno de cuatro vecinos.

Sean $R=\{(0,1),(0,-1),(1,0),(-1,0)\} ; a \in \mathbb{R} ; A_{R}:=\left\{a_{\tilde{r}} / a_{\tilde{r}}=a\right.$, $\tilde{r} \in R\} ; \eta:=\left\{\eta_{\tilde{s}} / \tilde{s} \in \mathbb{Z}^{2}\right\}$ un proceso $S W N$.

Se dice que $X=\left\{X_{\tilde{s}} / \tilde{s} \in \mathbb{Z}^{2}\right\}$ es un proceso SAR isotrópico para entorno de cuatro vecinos con proceso de innovación $\eta$, si:

1. $E\left(X_{\tilde{s}}\right)=0 \forall \tilde{s} \in \mathbb{Z}^{2}$.

2. $X_{\tilde{s}} \in \mathcal{L}^{2}(\Omega, \mathcal{F}, P, \mathbb{R}) \forall \tilde{s} \in \mathbb{Z}^{2}$.

3. $X_{\tilde{s}}=a \sum_{\tilde{r} \in R} X_{\tilde{s}-\tilde{r}}+\eta_{\tilde{s}}$.

Proposición 2.13.4. Consideremos la situación del ejemplo anterior. Si $|a|<\frac{1}{4}$, entonces $\exists X \in w-\mathcal{L}^{2}$ tal que $X$ es un SAR isotrópico para entorno de cuatro vecinos.

Ejemplo 2.13.5. Modelo SAR(1) factorizable. Sean $R=\{(0,1),(0,-1),(1,0),(-1,0)\} ; \alpha, \beta \in \mathbb{R}:|\alpha|<1$ y $|\beta|<1 ; A_{R}:=$ $\left\{a_{\tilde{r}} / \tilde{r} \in R\right.$ y $a_{(-1,0)}=\alpha, a_{(0,-1)}=\beta$ y $\left.a_{(-1,-1)}=\alpha \beta\right\} ; \eta:=\left\{\eta_{\tilde{s}} / \tilde{s} \in \mathbb{Z}^{2}\right\}$ un proceso $S W N$. Se dice que $X=\left\{X_{\tilde{s}} / \tilde{s} \in \mathbb{Z}^{2}\right\}$ es un proceso $\boldsymbol{S A R}(1)$ factorizable con proceso de innovaciones $\eta$, si:

1. $E\left(X_{\tilde{s}}\right)=0 \forall \tilde{s} \in \mathbb{Z}^{2}$.

2. $X_{\tilde{s}} \in \mathcal{L}^{2}(\Omega, \mathcal{F}, P, \mathbb{R}) \forall \tilde{s} \in \mathbb{Z}^{2}$. 
3. $X_{\tilde{s}}=\sum_{\tilde{r} \in R} a_{\tilde{r}} X_{\tilde{s}-\tilde{r}}+\eta_{\tilde{s}}=\alpha X_{\left(s_{1}-1, s_{2}\right)}+\beta X_{\left(s_{1}, s_{2}-1\right)}+\alpha \beta X_{\left(s_{1}-1, s_{2}-1\right)}+$ $\eta_{\tilde{s}} \operatorname{con} \tilde{s}=\left(s_{1}, s_{2}\right)$.

Notación 2.13.6. Sea $s \in \mathbb{Z}$. Con $B_{1}^{s} y B_{2}^{s}$ denotaremos las funciones definidas sobre $\mathbb{R}^{\mathbb{Z}^{2}}$ por:

$$
\begin{aligned}
& B_{1}^{s}(x)(\tilde{t}):=x\left(t_{1}-s, t_{2}\right) \\
& B_{2}^{s}(x)(\tilde{t}):=x\left(t_{1}, t_{2}-s\right)
\end{aligned}
$$

para todo $\tilde{t}=\left(t_{1}, t_{2}\right)$.

Notación 2.13.7. Consideremos el ejemplo anterior. Si X satisface 1), 2) y 3) del ejemplo entonces,

$$
\left(I-\alpha B_{1}^{1}\right)\left(\left(I-\beta B_{2}^{1}\right)(X)\right)=\eta,
$$

donde I es el operador identidad de $\mathbb{R}^{\mathbb{Z}^{2}}$. Este resultado justifica el nombre de "factorizable" dado a un proceso como el $X$.

Proposición 2.13.8. Sea $X$ un proceso SAR(1) factorizable como en el ejemplo. Entonces cualquiera sean $\left(s_{1}, s_{2}\right)$ y $\left(s_{1}^{\prime}, s_{2}^{\prime}\right)$ en $\mathbb{Z}^{2}$ :

$C_{X}^{0}\left(s_{1}-s_{1}^{\prime}, s_{2}-s_{2}^{\prime}\right)=\sigma_{X}^{2} \alpha^{\left|s_{1}-s_{1}^{\prime}\right|} \beta^{\left|s_{2}-s_{2}^{\prime}\right|}$ con $\sigma_{X}^{2}=\sigma_{\eta}^{2} \frac{1}{1-\alpha^{2}} \frac{1}{1-\beta^{2}} y$ $\sigma_{\eta}^{2}=\operatorname{Var}\left(\eta_{\tilde{s}}\right) \forall \tilde{s} \in S$.

Definición 2.13.9. Diremos que un sistema $\left(R, A_{R}, \eta\right)$ identifica un proceso $\boldsymbol{S A R}, X=\left\{X_{\tilde{s}} / \tilde{s} \in \mathbb{Z}^{2}\right\}$ si

i) $R \subset \mathbb{Z}^{2} \backslash\{(0,0)\}$ es finito no vacío.

ii) $A_{R}=\left\{a_{\tilde{r}} / \tilde{r} \in R\right\} \subset \mathbb{R}$.

iii) $\eta=\left\{\eta_{\tilde{s}} / \tilde{s} \in \mathbb{Z}^{2}\right\}$ es un proceso $S W N$.

iv) $X_{\tilde{s}}=\sum_{\tilde{r} \in R} a_{\tilde{r}} X_{\tilde{s}-\tilde{r}}+\eta_{\tilde{s}}$ en $\mathcal{L}^{2}(\Omega, \mathcal{F}, P, \mathbb{R})$.

v) Sea $\left(R^{*}, A_{R^{*}}, \eta^{*}\right)$ otro sistema que satisface $\left.i\right)$ - iv), entonces $R^{*}=R$, $A_{R^{*}}=A_{R}$ y $\eta_{\tilde{s}}^{*}=\eta_{\tilde{s}}$ en $\mathcal{L}^{2}(\Omega, \mathcal{F}, P, \mathbb{R})$ y $\forall \tilde{s} \in \mathbb{Z}^{2}$.

\subsection{Procesos autorregresivos condicionales estacionarios}

Proposición 2.14.1. Sea $O(\leq):=\left\{\left((a, b),\left(a^{\prime}, b^{\prime}\right)\right) \in \mathbb{Z}^{2} \times \mathbb{Z}^{2} / a<a^{\prime}\right.$ ó $b \leq b^{\prime}$ y $\left.a=a^{\prime}\right\}$.

Si $\left((a, b),\left(a^{\prime}, b^{\prime}\right)\right) \in O(\leq)$, entonces pondremos $(a, b) \leq\left(a^{\prime}, b^{\prime}\right) . L a$ relación $\leq$ entre elementos de $\mathbb{Z}^{2}$ es de orden total, llamado orden lexicográfico en $\mathbb{Z}^{2}$. 
De ahora en adelante entenderemos que $(a, b) \leq\left(a^{\prime}, b^{\prime}\right)$ entre elementos de $\mathbb{Z}^{2}$ se refiere al orden lexicográfico.

Definición 2.14.2. Sean: $L \subset \mathbb{Z}^{2} \backslash\{(0,0)\}$ finito, no vacío y simétrico de $\mathbb{Z}^{2}$ (esto es $\left.(a, b) \in L \Rightarrow(-a,-b) \in L\right) ; L^{+}:=\{\tilde{s} \in L / \tilde{0} \leq \tilde{s}\}$.

Diremos que $X=\left\{X_{\tilde{s}} / \tilde{s} \in \mathbb{Z}^{2}\right\}$ con $X_{\tilde{s}} \in \mathcal{L}^{2}$ y $E\left(X_{\tilde{s}}\right)=0 \forall \tilde{s} \in \mathbb{Z}^{2}$ es un proceso aleatorio L-markoviano $\operatorname{CAR}(L)$ si:

1. $X \in w-\mathcal{L}^{2}$.

2. $\sum_{\tilde{s} \in L} c_{\tilde{s}} X_{\tilde{t}-\tilde{s}}+e_{\tilde{t}}$ donde

(a) $c_{\tilde{s}} \in \mathbb{R}, c_{\tilde{s}}=c_{-\tilde{s}} \forall \tilde{s} \in L$.

(b) $e:=\left\{e_{\tilde{t}} / \tilde{t} \in \mathbb{Z}^{2}\right\}$ es un proceso con $E\left(e_{\tilde{t}}\right)=0 \forall \tilde{t} \in \mathbb{Z}^{2}$.

(c) $\operatorname{Cov}\left(e_{\tilde{t}}, X_{\tilde{s}}\right)=0 \forall \tilde{t} \neq \tilde{s} \in \mathbb{Z}^{2}$.

Definición 2.14.3. Sean $X=\left\{X_{\tilde{s}} / \tilde{s} \in \mathbb{Z}^{2}\right\}$ y $\hat{X}=\left\{\hat{X}_{\tilde{s}} / \tilde{s} \in \mathbb{Z}^{2}\right\}$ dos procesos sobre el mismo espacio medible. Diremos que $\hat{X}$ es un predictor lineal de $X$ si $\exists R \subset \mathbb{Z}^{2} \backslash\{(0,0)\}$ finito, no vacío; $A_{R}=\left\{a_{\tilde{r}} / \tilde{r} \in R\right\} \subset \mathbb{R}$ tales que

$$
\hat{X}_{t}=\sum_{\tilde{r} \in R} a_{\tilde{r}} X_{\tilde{t}-\tilde{r}} .
$$

Notación 2.14.4. Pondremos: $\mathcal{L}(X)=\{\hat{X} / \hat{X}$ es un predictor lineal de $X\}$.

Definición 2.14.5. Sea $X=\left\{X_{\tilde{s}} / \tilde{s} \in \mathbb{Z}^{2}\right\}$ un proceso con $X_{\tilde{s}}$ en $\mathcal{L}^{2}(\Omega, \mathcal{F}$, $P, \mathbb{R})$, diremos que $\hat{X}=\left\{\hat{X}_{\tilde{s}} / \tilde{s} \in \mathbb{Z}^{2}\right\} \in \mathcal{L}(X)$ es un predictor lineal óptimo de $X$ si:

$$
\left\|X_{\tilde{s}}-\hat{X}_{\tilde{s}}\right\|_{\mathcal{L}^{2}} \leq\left\|X_{\tilde{s}}-\tilde{X}_{\tilde{s}}\right\|_{\mathcal{L}^{2}} \forall \tilde{s} \in \mathbb{Z}^{2} \forall \tilde{X} \in \mathcal{L}(X)
$$

Proposición 2.14.6. Sea $X=\left\{X_{\tilde{s}} / \tilde{s} \in \mathbb{Z}^{2}\right\}$ un proceso $C A R(L)$. Para cada $\tilde{t} \in \mathbb{Z}^{2}$ sea

$$
\hat{X}_{\tilde{t}}:=\sum_{\tilde{s} \in L} c_{\tilde{s}} X_{\tilde{t}-\tilde{s}} .
$$

Entonces $\hat{X}=\left\{\hat{X}_{\tilde{s}} / \tilde{s} \in \mathbb{Z}^{2}\right\}$ es un predictor lineal óptimo de $X$.

Definición 2.14.7. Sean $X=\left\{X_{\tilde{s}} / \tilde{s} \in \mathbb{Z}^{2}\right\}$ y $\hat{X}=\left\{\hat{X}_{\tilde{s}} / \tilde{s} \in \mathbb{Z}^{2}\right\}$ dos procesos del mismo espacio medible. Diremos que $\hat{X}$ es un predictor de $X$ si $\exists R \subset \mathbb{Z}^{2} \backslash\{(0,0)\}$ finito, no vacío y $g: \mathbb{R}^{R} \rightarrow \mathbb{R}$ tal que para cada $\tilde{t} \in \mathbb{Z}^{2}$ se tiene

$$
\hat{X}_{\tilde{t}}=g\left(X_{\tilde{t}-R}\right)
$$


donde $X_{\tilde{t}-R}: \Omega \longrightarrow \mathbb{R}^{R}$ está definida por

$$
X_{\tilde{t}-R}(\omega)(\tilde{s})=X_{\tilde{t}-\tilde{s}}(\omega) \forall \omega \in \Omega, \forall \tilde{s} \in R .
$$

Notación 2.14.8. Pondremos $\mathcal{P}(X):=\{\hat{X} / \hat{X}$ es un predictor de $X\}$.

Definición 2.14.9. Sea $X=\left\{X_{\tilde{s}} / \tilde{s} \in \mathbb{Z}^{2}\right\}$ un proceso con $X_{\tilde{s}}$ en $\mathcal{L}^{2}(\Omega, \mathcal{F}$, $P, \mathbb{R})$, diremos que $\hat{X}=\left\{\hat{X}_{\tilde{s}} / \tilde{s} \in \mathbb{Z}^{2}\right\} \in \mathcal{P}(X)$ es un predictor óptimo de $X$ si:

$$
\left\|X_{\tilde{s}}-\hat{X}_{\tilde{s}}\right\|_{\mathcal{L}^{2}} \leq\left\|X_{\tilde{s}}-\tilde{X}_{\tilde{s}}\right\|_{\mathcal{L}^{2}} \forall \tilde{s} \in \mathbb{Z}^{2} \forall \tilde{X} \in \mathcal{P}(X)
$$

Proposición 2.14.10. Sea $X=\left\{X_{\tilde{s}} / \tilde{s} \in \mathbb{Z}^{2}\right\}$ un proceso $C A R(L)$ gaussiano. Para cada $\tilde{t} \in \mathbb{Z}^{2}$ sea

$$
\hat{X}_{\tilde{t}}:=\sum_{\tilde{s} \in L} c_{\tilde{s}} X_{\tilde{t}-\tilde{s}}
$$

Entonces $\hat{X}=\left\{\hat{X}_{\tilde{s}} / \tilde{s} \in \mathbb{Z}^{2}\right\}$ es un predictor óptimo de $X$.

Lema 2.14.11. Sea $X=\left\{X_{\tilde{s}} / \tilde{s} \in \mathbb{Z}^{2}\right\}$ un $w-\mathcal{L}^{2}$ tal que $C_{X}^{0}$ tiene densidad espectral $f_{C_{X}^{0}}$.

Sea $a \in l^{2}\left(\mathbb{Z}^{2}, \mathbb{R}\right)$. Sea $Y=\left\{Y_{\tilde{s}} / \tilde{s} \in \mathbb{Z}^{2}\right\}$ dado por

$$
Y_{\tilde{s}}=\sum_{\tilde{r} \in \mathbb{Z}^{2}} a_{\tilde{r}} X_{\tilde{s}-\tilde{r}}
$$

Entonces $Y$ es $w-\mathcal{L}^{2}$ tal que $C_{Y}^{0}$ tiene densidad espectral $f_{C_{Y}^{0}}$ dada por

$$
f_{C_{Y}^{0}}(\tilde{\lambda})=\left|\sum_{\tilde{r} \in \mathbb{Z}^{2}} a_{\tilde{r}} \exp (i\langle\tilde{\lambda}, \tilde{r}\rangle)\right|^{2} f_{C_{X}^{0}}(\tilde{\lambda})
$$

Proposición 2.14.12. Sea $\hat{X}=\left\{\hat{X}_{\tilde{s}} / \tilde{s} \in \mathbb{Z}^{2}\right\}$ un proceso $C A R(L)$. Supongamos que

i) $\sum_{\tilde{s} \in \mathbb{Z}^{2}}\left(C_{X}^{0}(\tilde{s})\right)^{2}<\infty$.

ii) Sea $P\left(e^{i \tilde{\lambda}}\right):=1-\sum_{\tilde{s} \in L^{+}} c_{\tilde{s}} \exp (i\langle\tilde{\lambda}, \tilde{s}\rangle)=1-2 \sum_{\tilde{s} \in L^{+}} c_{\tilde{s}} \cos (\langle\tilde{\lambda}, \tilde{s}\rangle), \tilde{\lambda} \in \mathbb{T}^{2}$, $\operatorname{con} P\left(e^{i \tilde{\lambda}}\right) \neq 0 \forall \tilde{\lambda} \in \mathbb{T}^{2}$.

Entonces: 
a) $C_{X}^{0}$ tiene densidad espectral dada por

$$
f_{C_{e}^{0}}(\tilde{\lambda})=\left(\frac{1}{2 \pi}\right)^{2} \sigma_{e}^{2} \frac{1}{P\left(e^{i \tilde{\lambda}}\right)}, \tilde{\lambda} \in \mathbb{T}^{2}
$$

b) Se cumple:

$$
\operatorname{Cov}\left(e_{\tilde{t}}, e_{\tilde{t}+\tilde{s}}\right)=\left\{\begin{array}{l}
\sigma_{e}^{2} \quad \text { si } \quad \tilde{s}=0 \\
-\sigma_{e} c_{\tilde{s}} \quad \text { si } \quad \tilde{s} \in L \\
0 \quad \text { c.c. }
\end{array}\right.
$$

Proposición 2.14.13. Sean: $L \subset \mathbb{Z}^{2} \backslash\{(0,0)\}$ finito, no vacío y simétrico; $L^{+}:=\{\tilde{s} \in L / \tilde{0} \leq \tilde{s}\} ;\left\{c_{\tilde{s}} / \tilde{s} \in \mathbb{Z}^{2}\right\}$ satisfaciendo:

$c_{\tilde{s}}=c_{-\tilde{s}} \forall \tilde{s} \in L$.

$c_{\tilde{s}}=0 \forall \tilde{s} \notin L$.

$\sigma_{e}>0 ; \widetilde{e}=\left\{e_{\tilde{t}} / \tilde{t} \in \mathbb{Z}^{2}\right\}$ es un proceso $w-\mathcal{L}^{2}$ centrado con

$$
C_{e}^{0}(\tilde{s})=\left\{\begin{array}{l}
\sigma_{e}^{2} \quad \text { si } \quad \tilde{s}=0 \\
-\sigma_{e} c_{\tilde{s}} \quad \text { si } \quad \tilde{s} \in L \\
0 \quad \text { c.c. }
\end{array}\right.
$$

Para cada $\tilde{\lambda} \in \mathbb{T}^{2}$ sea $P\left(e^{i \tilde{\lambda}}\right):=1-2 \sum_{\tilde{s} \in L^{+}} c_{\tilde{s}} \cos (\langle\tilde{\lambda}, \tilde{s}\rangle) \operatorname{con} P\left(e^{i \tilde{\lambda}}\right) \neq 0$ $\forall \tilde{\lambda} \in \mathbb{T}^{2}$.

Entonces:

a) $C_{e}^{0}$ tiene densidad espectral dada por

$$
f_{C_{X}^{0}}(\tilde{\lambda})=\left(\frac{\sigma_{e}}{2 \pi}\right)^{2} P\left(e^{i \tilde{\lambda}}\right), \tilde{\lambda} \in \mathbb{T}^{2}
$$

b) Existe $X=\left\{X_{\tilde{s}} / \tilde{s} \in \mathbb{Z}^{2}\right\}$ un $w-\mathcal{L}^{2}$ tal que

$$
X_{\tilde{t}}:=\sum_{\tilde{s} \in L} c_{\tilde{s}} X_{\tilde{t}-\tilde{s}}+e_{\tilde{t}} \quad \forall \tilde{t} \in \mathbb{Z}^{2} .
$$

Esto es: $X$ es un proceso $C A R(L)$ con $\widetilde{e}$ como proceso residual.

Proposición 2.14.14. Sea $X=\left\{X_{\tilde{s}} / \tilde{s} \in \mathbb{Z}^{2}\right\}$ un proceso SAR tal que

$$
P\left(e^{i \tilde{\lambda}}\right):=1-\sum_{\tilde{s} \in R} a_{\tilde{s}} \exp (i\langle\tilde{\lambda}, \tilde{s}\rangle) \neq 0, \tilde{\lambda} \in \mathbb{T}^{2} .
$$

Sea $\tilde{R}:=\left\{\left(\tilde{r}_{1}, \tilde{r}_{2}\right) / \tilde{r}_{1} \in R, \tilde{r}_{2} \in R\right.$ y $\left.\tilde{r}_{1}>\tilde{r}_{2}\right\}$ y sea $\equiv$ la relación de equivalencia en $\tilde{R}$ dada por $\left(\tilde{r}_{1}, \tilde{r}_{2}\right) \equiv\left(\tilde{r}_{1}^{\prime}, \tilde{r}_{2}^{\prime}\right)$ si $\tilde{r}_{1}-\tilde{r}_{2}=\tilde{r}_{1}^{\prime}-\tilde{r}_{2}^{\prime}$. 
Sea: $R_{0}=\tilde{R} / \equiv y Q: \tilde{R} \mapsto R_{0}$ la proyección canónica. Para cada $J \in R_{0} \operatorname{sea}\left(\tilde{r}_{1}^{J}, \tilde{r}_{2}^{J}\right) \in \tilde{R}$ tal que

$Q\left(\left(\tilde{r}_{1}^{J}, \tilde{r}_{2}^{J}\right)\right)=J$ y $\tilde{r}_{1}^{J} \leq \tilde{r}_{1}, \tilde{r}_{2}^{J} \leq \tilde{r}_{2} \quad \forall\left(\tilde{r}_{1}, \tilde{r}_{2}\right) \in \tilde{R}$ tal que $Q\left(\left(\tilde{r}_{1}, \tilde{r}_{2}\right)\right)=J$

Sea $R_{1}:=\left\{\tilde{r}_{1}^{J}-\tilde{r}_{2}^{J} / J \in R_{0}\right\}$ y sean $R_{1}^{*}=R_{1} \backslash R ; L:=R \cup(-R) \cup$ $R_{1}^{*} \cup\left(-R_{1}^{*}\right)$.

Sea $\tilde{s} \in \mathbb{Z}^{2} \Rightarrow c_{\tilde{s}}:=0$ si $\tilde{s} \notin L$.

$$
c_{\tilde{s}}=\left\{\begin{array}{c}
a_{\tilde{s}} /\left(1+\sum_{\left(\tilde{r}_{1}, \tilde{r}_{2}\right) \in J(\tilde{s})} a_{\tilde{r}_{1}} a_{\tilde{r}_{2}}\right) \quad \text { si } \quad \tilde{s} \in R \backslash R_{1} \\
\frac{1}{1+\sum_{\tilde{t} \in R} a_{\tilde{t}}^{2}}\left(a_{\tilde{s}}-\sum_{\left(\tilde{r}_{1}, \tilde{r}_{2}\right) \in J(\tilde{s})} a_{\tilde{r}_{1}} a_{\tilde{r}_{2}}\right)
\end{array}\right.
$$

siendo $\tilde{s}=\tilde{r}_{1}^{J}-\tilde{r}_{2}^{J}, J \in R_{0}, y J(\tilde{s}):=\left\{\left(\tilde{r}_{1}, \tilde{r}_{2}\right) \in \tilde{R} / Q\left(\left(\tilde{r}_{1}, \tilde{r}_{2}\right)\right)=J\right\}$.

$$
c_{\tilde{s}}=-\frac{1}{1+\sum_{\tilde{t} \in R} a_{\tilde{t}}^{2}} \sum_{\left(\tilde{r}_{1}, \tilde{r}_{2}\right) \in J(\tilde{s})} a_{\tilde{r}_{1}} a_{\tilde{r}_{2}} \quad \text { si } \quad \tilde{s} \in R_{1}^{*} .
$$

Finalmente, definimos $c_{\tilde{s}}=c_{-\tilde{s}}$ si $\tilde{s} \in(-R) \cup\left(-R_{1}^{*}\right)$.

Entonces

$$
\begin{array}{r}
f_{C_{X}^{0}}(\tilde{\lambda})=\left(\frac{1}{2 \pi}\right)^{2} \frac{\frac{\sigma_{\eta}^{2}}{1+\sum_{\tilde{t} \in R} a_{\tilde{t}}^{2}}}{\mathcal{C}(\exp (i \tilde{\lambda}))} \\
\operatorname{con} \mathcal{C}(\exp (i \tilde{\lambda})):=1-\sum_{\tilde{s} \in L} c_{\tilde{s}} \exp (i\langle\tilde{\lambda}, \tilde{s}\rangle) .
\end{array}
$$

Luego por la Proposición 2.14.13 existe $Y=\left\{Y_{\tilde{s}} / \tilde{s} \in \mathbb{Z}^{2}\right\}$ tal que

$$
Y_{\tilde{t}}:=\sum_{\tilde{s} \in L} c_{\tilde{s}} Y_{\tilde{t}-\tilde{s}}+e_{\tilde{t}} \quad \forall \tilde{t} \in \mathbb{Z}^{2}
$$

donde $\widetilde{e}=\left\{e_{\tilde{t}} / \tilde{t} \in \mathbb{Z}^{2}\right\}$ es un proceso $w-\mathcal{L}^{2}$ con

$$
C_{e}^{0}(\tilde{s})=\left\{\begin{array}{lll}
\sigma_{e}^{2} & \text { si } & \tilde{s}=0 \\
-\sigma_{e} c_{\tilde{s}} & \text { si } & \tilde{s} \in L . \\
0 & \text { c.c. }
\end{array} .\right.
$$

siendo $\sigma_{e}^{2}:=\frac{\sigma_{\eta}^{2}}{1+\sum_{\dot{t} \in R} a_{\hat{t}}^{2}}$.

Esto es: $Y$ es un proceso $C A R(L)$ con $\widetilde{e}$ como proceso residual y tal que $C_{Y}^{0}(\tilde{t})=C_{X}^{0}(\tilde{t}) \forall \tilde{t} \in \mathbb{Z}^{2}$. 
Proposición 2.14.15. Sean: $X=\left\{X_{s} / s \in \mathbb{Z}\right\}$ un $w-\mathcal{L}^{2} ; l>1$ entero;

$$
L:=\{ \pm 1, \ldots, \pm l\} \text {. }
$$

Para cada $s \in L$ sea $c_{s} \in \mathbb{R}$ tales que $c_{s}=c_{-s} \forall s \in L$ y $1-$ $2 \sum_{j=1}^{l} c_{j} \cos (j \lambda)>0 \forall \lambda \in(-\pi, \pi]$.

Supongamos que $X$ es un $C A R(L)$ con coeficientes $\left\{c_{s} / s \in L\right\}$ y con proceso de residuos $\widetilde{e}=\left\{e_{s} / s \in \mathbb{Z}\right\} ;$ esto es, se cumple

$$
X_{\tilde{t}}:=\sum_{s \in L} c_{s} X_{t-s}+e_{t} \quad \forall t \in \mathbb{Z}
$$

donde $\widetilde{e}=\left\{e_{t} / t \in \mathbb{Z}\right\}$ es un proceso $w-\mathcal{L}^{2}$ tal que

$$
\operatorname{Cov}\left(e_{t}, e_{t+s}\right)=\left\{\begin{array}{llll}
\sigma_{e}^{2} & \text { si } & s=0 \\
-\sigma_{e} c_{s} & \text { si } & s \in L
\end{array}\right.
$$

$\operatorname{con} \sigma_{e}>0$.

Entonces existen: $a_{1}, \ldots, a_{l}$ en $\mathbb{C}$ tales que si $\eta:=\left\{\eta_{s} / s \in \mathbb{Z}\right\}$ es un proceso $S W N$ e $Y=\left\{Y_{s} / s \in \mathbb{Z}\right\}$ es un proceso satisfaciendo:

$$
Y_{t}:=\sum_{s=1}^{l} a_{s} Y_{t-s}+e_{t} \quad \forall t \in \mathbb{Z},
$$

entonces

$$
C_{Y}^{0}(t)=C_{X}^{0}(t) \quad \forall t \in \mathbb{Z}
$$

Esta proposición junto con la anterior nos dice que: en dimensión 1 los conceptos de proceso $S A R$ y CAR son equivalentes.

El siguiente ejemplo muetra un $C A R(L)$ con dimensión 2 que no admite una representación $S A R$.

Ejemplo 2.14.16. Sean $L=\{(1,0),(0,1),(-1,0),(0,-1)\} ; c>0 ; X=$ $\left\{X_{\tilde{s}} / \tilde{s} \in \mathbb{Z}^{2}\right\}$ tal que $E\left(X_{\tilde{s}}\right)=0 \forall \tilde{s} \in \mathbb{Z}^{2}$ y

$$
X_{\tilde{t}}=c \sum_{\tilde{s} \in L} X_{\tilde{t}-\tilde{s}}+e_{\tilde{t}} \quad \forall \tilde{t} \in \mathbb{Z}^{2},
$$

donde $\widetilde{e}=\left\{e_{\tilde{t}} / \tilde{t} \in \mathbb{Z}^{2}\right\}$ es un proceso $w-\mathcal{L}^{2}$ satisfaciendo

$$
\operatorname{Cov}\left(e_{\tilde{t}}, e_{\tilde{t}+\tilde{s}}\right)=\left\{\begin{array}{lll}
\sigma_{e}^{2} & \text { si } & \tilde{s}=0 \\
-\sigma_{e} c_{\tilde{s}} & \text { si } & \tilde{s} \in L \\
0 & \text { c.c. }
\end{array}\right.
$$

$\operatorname{con} \sigma_{e}^{2}:=\frac{(2 \pi)^{2}}{c}$.

Entonces no existe $Y=\left\{Y_{\tilde{s}} / \tilde{s} \in \mathbb{Z}^{2}\right\}$ que sea SAR y tal que $f_{C_{X}^{0}}(\tilde{\lambda})=$ $f_{C_{Y}^{0}}(\tilde{\lambda}) \quad \forall \tilde{\lambda} \in \mathbb{T}^{2}$. 
Definición 2.14.17. Sean: $c \in l^{2}\left(\mathbb{Z}^{2}\right) ; \eta=\left\{\eta_{\tilde{s}} / \tilde{s} \in \mathbb{Z}^{2}\right\}$ un proceso $S W N$ $\operatorname{con} \sigma_{\eta}^{2}:=E\left(\eta_{\tilde{s}}^{2}\right) \forall \tilde{s} \in \mathbb{Z}^{2}$.

Diremos que $X=\left\{X_{\tilde{s}} / \tilde{s} \in \mathbb{Z}^{2}\right\}$ es un proceso de medias móviles de orden $\infty$ sobre $\mathbb{Z}^{2}$ con coeficiente c y proceso de innovación $\eta$ si

$$
X_{\tilde{t}}=\sum_{\tilde{s} \in \mathbb{Z}^{2}} c_{\tilde{s}} \eta_{\tilde{t}-\tilde{s}} \quad \forall \tilde{t} \in \mathbb{Z}^{2} .
$$

En símbolos: $X \in M A(\infty, c, \eta)$.

Proposición 2.14.18. Sean: $c \in l^{2}\left(\mathbb{Z}^{2}\right) ; \eta=\left\{\eta_{\tilde{s}} / \tilde{s} \in \mathbb{Z}^{2}\right\}$ un proceso $S W N$. Entonces existe $X \in M A(\infty, c, \eta)$.

Definición 2.14.19. Sea $c: \mathbb{Z}^{2} \mapsto \mathbb{R}$. Llamaremos soporte de $c$ a:

$$
\operatorname{Sop}(c):=\left\{\tilde{s} \in \mathbb{Z}^{2} / c(\tilde{s}) \neq 0\right\} .
$$

Definición 2.14.20. Sea $X \in M A(\infty, c, \eta)$. Diremos que $X$ es $M A d e$ orden finito si $\#(\operatorname{Sop}(c))<\infty$.

Nota 2.14.21. Sea $X \in M A(\infty, c, \eta)$. Si $X$ es $M A$ de orden finito, entonces $C_{X}^{0}(\tilde{s})=0$ para todo $\tilde{s} \notin(\operatorname{Sop}(c)-\operatorname{Sop}(c))$.

Proposición 2.14.22. Sea $X=\left\{X_{s} / s \in \mathbb{Z}\right\}$ un $w-\mathcal{L}^{2}$ centrado con $\#\left(\operatorname{Sop}\left(C_{X}^{0}\right)\right)<\infty$. Entonces existen $c \in l^{2}(\mathbb{Z}) ; \eta=\left\{\eta_{\tilde{s}} / \tilde{s} \in \mathbb{Z}\right\}$ un proceso $S W N$ tal que $X \in M A(\infty, c, \eta)$ y es de orden finito.

Nota 2.14.23. La proposición anterior en general no es cierta cuando consideramos procesos en $\mathbb{Z}^{2}$. En efecto. Sea $X=\left\{X_{\tilde{s}} / \tilde{s} \in \mathbb{Z}^{2}\right\}$ tal que

$$
\begin{gathered}
E\left(X_{\left(t_{1}, t_{2}\right)} X_{\left(t_{1}-1, t_{2}\right)}\right)=E\left(X_{\left(t_{1}, t_{2}\right)} X_{\left(t_{1}+1, t_{2}\right)}\right) \\
=E\left(X_{\left(t_{1}, t_{2}\right)} X_{\left(t_{1}, t_{2}-1\right)}\right)=E\left(X_{\left(t_{1}, t_{2}\right)} X_{\left(t_{1}, t_{2}+1\right)}\right)=\varrho \neq 0 \\
y c_{X}^{0}(\tilde{s})=0 \forall \tilde{s} \notin\{(0,1),(0,-1),(0,0),(1,0),(-1,0)\} .
\end{gathered}
$$

Procediendo en forma similar a lo realizado en el ejemplo anterior se puede probar que no existe $c \in l^{2}\left(\mathbb{Z}^{2}, \mathbb{C}\right) ; \eta=\left\{\eta_{\tilde{s}} / \tilde{s} \in \mathbb{Z}^{2}\right\}$ un proceso $S W N$ y tal que $X \in M A(\infty, c, \eta)$.

\subsubsection{Ejemplos de aplicación de la Proposición 2.14.14}

Ejemplo 2.14.24. SAR causal.

Sean $R=\{(1,0),(0,1)\} ; a_{(1,0)}:=\alpha ; a_{(0,1)}:=\beta ; X_{\tilde{t}}=\alpha X_{\tilde{t}-(1,0)}+$ $\beta X_{\tilde{t}-(0,1)}+\eta_{\tilde{t}}, \forall \tilde{t} \in \mathbb{Z}^{2}$ y $\eta=\left\{\eta_{\tilde{s}} / \tilde{s} \in \mathbb{Z}^{2}\right\}$ un proceso $S W N$.

Veamos su representacióm como CAR(L) usando la Proposición 2.14.14. 
Sean:

$$
\begin{gathered}
R^{1} \quad: \quad=\{(1,-1)\} \\
L \quad: \quad=R \cup(-R) \cup R^{1} \cup\left(-R^{1}\right) \\
=\{(0,1),(0,-1),(-1,1),(1,-1),(1,0),(-1,0)\} ; \\
c_{(0,1)}:=\frac{a_{(0,1)}}{1+a_{(0,1)}^{2}+a_{(1,0)}^{2}}=\frac{\beta}{1+\alpha^{2}+\beta^{2}} \quad c_{(1,0)}:=\frac{\alpha}{1+\alpha^{2}+\beta^{2}} \\
c_{(0,-1)}=c_{(1,0)} y c_{(-1,0)}=c_{(1,0)} \\
c_{(-1,1)}=c_{(1,-1)}=\frac{\alpha \beta}{1+\alpha^{2}+\beta^{2}} . \\
\text { Luego: } \\
X_{\tilde{t}}=\frac{1}{1+\alpha^{2}+\beta^{2}}\left(\alpha\left(X_{\tilde{t}-(1,0)}+X_{\tilde{t}-(-1,0)}\right)+\beta\left(X_{\tilde{t}-(0,1)}+X_{\tilde{t}-(0,-1)}\right)+\right. \\
\left.\alpha \beta\left(X_{\tilde{t}-(1,-1)}+X_{\tilde{t}-(-1,1)}\right)\right)+e_{\tilde{t}} \text { con } \widetilde{e}=\left\{e_{\tilde{t}} / \tilde{t} \in \mathbb{Z}^{2}\right\} \text { proceso centrado con } \\
\text { momento de } 2^{o} \text { orden finito y tal que }
\end{gathered}
$$

$$
\begin{aligned}
& E\left(X_{\tilde{t}} e_{\tilde{t}}\right)=E\left(e_{\tilde{t}}^{2}\right):=\sigma_{e}^{2} \quad \forall \tilde{t} \in \mathbb{Z}^{2} \\
& E\left(X_{\tilde{s}} e_{\tilde{t}}\right)=0 \quad \text { si } \tilde{s} \neq \tilde{t} \forall \tilde{t}, \tilde{s} \in \mathbb{Z}^{2} .
\end{aligned}
$$

Como ya probamos, se tiene:

$$
C_{e}^{0}(\tilde{s})=\left\{\begin{array}{l}
\sigma_{e}^{2} \quad \text { si } \quad \tilde{s}=0 \\
-\sigma_{e} c_{\tilde{s}} \text { si } \quad \tilde{s} \in L \\
0 \quad \text { c.c. }
\end{array}\right.
$$

Ejemplo 2.14.25. SAR no causal.

Sea $X_{\tilde{t}}=a\left(X_{\tilde{t}-(-1,0)}+X_{\tilde{t}-(1,0)}\right)+b\left(X_{\tilde{t}-(0,1)}+X_{\tilde{t}-(0,-1)}\right)+\eta_{\tilde{t}}$ para todo $\tilde{t} \in \mathbb{Z}^{2}$ con $\eta=\left\{\eta_{\tilde{s}} / \tilde{s} \in \mathbb{Z}^{2}\right\}$ un $S W N$.

Aplicando la Proposición 2.14.14 se obtiene la siguiente representación $C A R$ de $X=\left\{X_{\tilde{t}} / \tilde{t} \in \mathbb{Z}^{2}\right\}$ :

$$
X_{\tilde{t}}=\sum_{\tilde{s} \in L} c_{\tilde{s}} X_{\tilde{t}-\tilde{s}}+e_{\tilde{t}} \quad \forall \tilde{t} \in \mathbb{Z}^{2}
$$

con

$$
\begin{aligned}
L & :=L_{1} \cup L_{2}, \\
L_{1}: & =\{(0,1),(0,-1),(-1,1),(1,-1),(1,0),(-1,0)\}, \\
L_{2}: & =\{(1,1),(-1,-1),(2,0),(-2,0),(0,2),(0,-2)\} .
\end{aligned}
$$

Los coeficientes $\left\{c_{\tilde{s}} / \tilde{s} \in L\right\}$ resultan ser:

$$
\begin{aligned}
& c_{(1,0)}=c_{(-1,0)}=a K ; c_{(0,1)}=c_{(0,-1)}=b K \\
& c_{(1,1)}=c_{(-1,-1)}=-2 a b K=c_{(1,-1)}=c_{(-1,1)} \\
& c_{(2,0)}=c_{(-2,0)}=-a^{2} K ; c_{(0,2)}=c_{(0,-2)}=-b^{2} K ;
\end{aligned}
$$


$K=\left(1+2 a^{2}+2 b^{2}\right)^{-1}$.

$\widetilde{e}=\left\{e_{\tilde{t}} / \tilde{t} \in \mathbb{Z}^{2}\right\}$ proceso centrado tal que

$$
\begin{aligned}
E\left(e_{\tilde{t}}^{2}\right) & :=\sigma_{e}^{2}>0 \\
\operatorname{Cov}\left(e_{\tilde{t}}, e_{\tilde{t}+\tilde{s}}\right) & =\left\{\begin{array}{lll}
-\sigma_{e} c_{\tilde{s}} & \text { si } & \tilde{s} \in L, \tilde{t} \in \mathbb{Z}^{2} \\
0 & \text { si } & \tilde{s} \notin L, \tilde{t} \in \mathbb{Z}^{2} .
\end{array}\right.
\end{aligned}
$$

Ejemplo 2.14.26. SAR factorizante.

$X_{\tilde{t}}=\alpha X_{\tilde{t}-(1,0)}+\beta X_{\tilde{t}-(0,1)}-\alpha \beta X_{\tilde{t}-(-1,1)}+\eta_{\tilde{t}}, \forall \tilde{t} \in \mathbb{Z}^{2} y \eta=\left\{\eta_{\tilde{s}} / \tilde{s} \in \mathbb{Z}^{2}\right\}$ un proceso $S W N$.

Aplicando la Proposición 2.14.14 se obtiene la siguiente representación CAR de $X=\left\{X_{\tilde{t}} / \tilde{t} \in \mathbb{Z}^{2}\right\}$ :

$$
X_{\tilde{t}}=\sum_{\tilde{s} \in L} c_{\tilde{s}} X_{\tilde{t}-\tilde{s}}+e_{\tilde{t}} \quad \forall \tilde{t} \in \mathbb{Z}^{2}
$$

con $L=\{(0,1),(0,-1),(-1,1),(1,-1),(1,0),(-1,0),(1,1),(-1,-1)\}$. Los coeficientes $\left\{c_{\tilde{s}} / \tilde{s} \in L\right\}$ resultan ser:

$$
\begin{aligned}
& c_{(1,0)}=c_{(-1,0)}=\alpha\left(1+\alpha^{2}\right)^{-1} \\
& c_{(0,1)}=c_{(0,-1)}=\beta\left(1+\beta^{2}\right)^{-1} \\
& c_{(1,1)}=c_{(-1,-1)}=-\alpha \beta K=c_{(1,-1)}=c_{(-1,1)} ; \\
& K=\left(1+\alpha^{2}\right)^{-1}\left(1+\beta^{2}\right)^{-1} . \\
& \widetilde{e}=\left\{e_{\tilde{t}} / \tilde{t} \in \mathbb{Z}^{2}\right\} \text { como en los ejemplos anteriores. }
\end{aligned}
$$

\subsection{Modelos autorregresivos no-estacionarios sobre redes finitas}

Sea $S=\left\{s_{1}, . ., s_{n}\right\}$ con $n \geq 2$ entero. Sea $I: S \mapsto\{1, \ldots, n\}$ biyectiva.

Sea $X=\left\{X_{s} / s \in S\right\}$.

Definimos $X^{*}$ vector aleatorio con valores en $\mathbb{R}^{n}$ dado por:

$$
X_{s_{i}}=X_{I\left(s_{i}\right)}^{*} \quad 1 \leq i \leq n .
$$

Suponemos que $X$ es centrado. Luego:

$$
E\left(X^{*}\right)=0 \in \mathbb{R}^{n} .
$$

Con $\Sigma\left(X^{*}\right)$ denotamos la matriz de covarianza de $X^{*}$.

En esta Sección supondremos que todos las variables que trabajaremos tienen momento de $2^{\circ}$ orden finito. 
Definición 2.15.1. Sea $\varepsilon=\left\{\varepsilon_{s} / s \in S\right\}$ un proceso. Se dice que $\varepsilon$ es un ruido si $E\left(\varepsilon_{s}\right)=0 \forall s \in S$ y $\Sigma\left(\varepsilon^{*}\right)=\sigma_{\varepsilon} I d_{n}$ donde Id $d_{n}$ es la matriz identidad $n \times n$ y $\sigma_{\varepsilon}>0$.

Definición 2.15.2. Sean $X=\left\{X_{s} / s \in S\right\}$ un proceso $y \varepsilon=\left\{\varepsilon_{s} / s \in S\right\}$ un ruido. Diremos que:

a) $X$ admite una representación $\boldsymbol{A} \boldsymbol{R}$ con innovación $\varepsilon$ si $\exists A$ matriz $n \times n$ (real o compleja) tal que $A X^{*}=\varepsilon^{*}$.

b) $X$ admite una representación $M A$ con innovación $\varepsilon$ si $\exists B$ matriz $n \times n$ tal que $X^{*}=B \varepsilon^{*}$.

c) $X$ admite una representación $\boldsymbol{A} \boldsymbol{R} \boldsymbol{M A}$ con innovación $\varepsilon$ si $\exists A, B$ matriz $n \times n$ tal que $A X^{*}=B \varepsilon^{*}$.

Nota 2.15.3. Continuando la definición anterior. Relaciones entre $§\left(X^{*}\right)$ y $\Sigma\left(\varepsilon^{*}\right)$.

a) $A X^{*}=\varepsilon^{*}$ y A inversible $\Rightarrow \nwarrow\left(X^{*}\right)=A^{-1} \varepsilon^{*}\left(A^{-1}\right)^{t}$.

b) $X^{*}=B \varepsilon^{*} \Rightarrow \Sigma\left(X^{*}\right)=B \not\left(\varepsilon^{*}\right) B^{t}$.

c) $A X^{*}=B \varepsilon^{*}$ y $A$ inversible $\Rightarrow \nwarrow\left(X^{*}\right)=A^{-1} B \not\left(\varepsilon^{*}\right) B^{t}\left(A^{-1}\right)^{t}$.

\section{Nota 2.15.4. Descomposición de Cholesky}

Si $\Sigma\left(X^{*}\right)$ es definida no negativa, entonces existe una matriz L triangular inferior tal que

$$
\Sigma\left(X^{*}\right)=L L^{t}
$$

En el caso complejo: Si $\Sigma\left(X^{*}\right)$ es hermitiana $\left(\Sigma\left(X^{*}\right)=\left(\Sigma\left(X^{*}\right)\right)^{*}\right.$ transpuesta conjugada de $\left.\Sigma\left(X^{*}\right)\right)$ entonces $\Sigma\left(X^{*}\right)=L L^{*}$ donde $L^{*}$ es la transpuesta conjugada de $L$.

Si $\Sigma\left(X^{*}\right)$ es definida positiva, entonces la diagonal de $L$ tiene todas sus valores mayores que 0 .

\subsubsection{Representación SAR-local uniparamétrica}

Sea $W$ una matriz $n \times n$ tal que $W_{(i, i)}=0 \forall i=1, \ldots, n$.

Definición 2.15.5. Sean $X=\left\{X_{s} / s \in S\right\}$ un proceso y $\varepsilon=\left\{\varepsilon_{s} / s \in S\right\}$ un ruido. Diremos que $X$ admite una representación $S A R$-local uniparamétrica con matriz de pesos $W$ e innovación $\varepsilon$ si $\exists \varrho \in \mathbb{R}$ tal que:

$$
X^{*}=\varrho W X^{*}+\varepsilon^{*}
$$




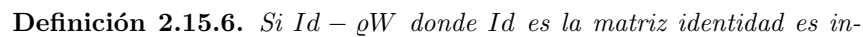
vertible, entonces $X^{*}$ está bien definida, en el sentido que si $Y=\left\{Y_{s} /\right.$ $s \in S\}$ es otro proceso tal que

$$
Y^{*}=\varrho W Y^{*}+\varepsilon^{*}
$$

entonces $X^{*}=Y^{*}$.

\subsubsection{Representación CAR-Markov}

Definición 2.15.7. Sean $X=\left\{X_{s} / s \in S\right\}$ un proceso y $e=\left\{e_{s} / s \in S\right\}$ un ruido con $E\left(e_{s}\right)=0, \operatorname{Var}\left(e_{s}\right)>0 \forall s \in S$. Diremos que $X$ admite una representación $\boldsymbol{C A R}$ con matriz de coeficientes $C=\left[c_{s, t}\right]_{s \in S, t \in S} \boldsymbol{y}$ proceso de innovaciones dado por e si

i) $c_{s, s}=0 \forall s \in S$.

ii) $X^{*}=C X^{*}+e^{*}$.

iii) $E\left(X_{t} e_{s}\right)=0$ si $s \neq t$.

Proposición 2.15.8. Si $X$ admite una representación CAR con matriz de coeficientes $C$ y proceso de innovaciones dado por e entonces:

a) $(I d-C) \Sigma\left(X^{*}\right)=\mathcal{D}:=\operatorname{Diagonal}\left(\left(\operatorname{Var}\left(e_{s}\right)\right)_{s \in S}\right)$.

b) $\Sigma\left(X^{*}\right)$ es invertible si y solo si $\mathcal{D}^{-1}(I d-C)$ es simétrica definida positiva.

Luego debe cumplirse:

$$
c_{s, t} \operatorname{Var}\left(e_{t}\right)=c_{t, s} \operatorname{Var}\left(e_{s}\right) .
$$

Nota 2.15.9. Sean $X$ e $Y$ procesos Gaussianos sobre $S$ tales que

$$
\begin{aligned}
& X^{*}=C X^{*}+e \\
& Y^{*}=C Y^{*}+e .
\end{aligned}
$$

Entonces $X \stackrel{\mathcal{D}}{=} Y$.

\subsubsection{Procesos de Gauss-Markov}

Sean:

i) $X=\left\{X_{s} / s \in S\right\}$ un proceso Gaussiano tal que

$$
X^{*} \sim \mathcal{N}_{n}(\tilde{\mu}, \Sigma) \quad \text { con } \Sigma \text { definida positiva; }
$$


ii) $\mathcal{V}=\left\{V_{s} / s \in S\right\}$ un sistema de vencindades sobre $S$, esto es:
1. $V_{s} \neq \emptyset \forall s \in S$,
2. $s \notin V_{s}, \forall s \in S$,
3. $s \in V_{t} \Leftrightarrow t \in V_{s}$,
4. $S=\bigcup_{s \in S} V_{s}$.

Al par $\mathcal{G}=(S, \mathcal{V})$ lo llamaremos grafo sobre $S$.

Sea

$$
Q=\left[q_{(s, t)}\right]_{(s, t) \in S \times S}:=\Sigma^{-1} .
$$

Supongamos que $q_{(s, t)}=0$ si $s \neq t$ y $s \notin V_{t}$

Proposición 2.15.10. Para cada $s \in S$ sea $\gamma_{\{s\}}^{0}: \mathcal{B}_{1} \times \mathbb{R}^{S} \rightarrow[0,1]$ dada por:

$$
\gamma_{\{s\}}^{0}(A \mid x)=\int_{A} \phi\left(y ; \mu\left(x_{S \backslash\{s\}}\right), \sigma\left(x_{S \backslash\{s\}}\right)\right) d y
$$

con

$$
\begin{aligned}
\phi(y ; a, b) & :=\frac{1}{\sqrt{2 \pi b}} \exp \left(-\frac{1}{2} \frac{(x-a)^{2}}{b^{2}}\right) \quad a \in \mathbb{R}, b>0 \\
\mu\left(x_{S \backslash\{s\}}\right) & :=\mu_{s}-\frac{1}{q_{(s, s)}} \sum_{t \in V_{s}} q_{(t, s)}\left(x_{t}-\mu_{t}\right) \\
\sigma\left(x_{S \backslash\{s\}}\right) & :=\frac{1}{q(s, s)} .
\end{aligned}
$$

Entonces:

$$
\gamma_{\{s\}}^{0}(\cdot \mid x) \in P\left(X_{s}^{*} \mid X_{t}^{*}=x_{t}, t \neq s\right) .
$$

Nota 2.15.11. Sea $Y=\left\{Y_{s} / s \in S\right\}$ dada por

$$
Y_{s}=X_{s}-\mu_{s} \quad \forall s \in S \text {. }
$$

Sean:

$$
\begin{gathered}
{[Q](s, t):=\left\{\begin{array}{cc}
0 & \text { si } s=t \\
q_{(s, t)} & \text { si } s \neq t
\end{array}\right.} \\
\text { Diag }:=\operatorname{Diagonal}\left(q_{(s, s)} / s \in S\right) .
\end{gathered}
$$

Sea $e=\left\{e_{s} / s \in S\right\}$ dado por:

$$
\begin{gathered}
e_{s}=Y_{s}-\frac{1}{q_{(s, s)}} \sum_{t \in V_{s}} q_{(s, t)} Y_{t} \\
y \operatorname{Var}\left(e_{s}\right):=\frac{1}{q_{(s, s)}}
\end{gathered} \quad \forall s \in S .
$$

Entonces $Y$ admite una representación $C A R$ con matriz de coeficientes

$$
C=-\operatorname{Diag}^{-1} \cdot[Q]
$$

$y$ proceso de innovaciones dado por $e$.

Si se cumple que $q_{(s, t)} \neq 0$ si y sólo si $t \in V_{s}$ cuando $s \neq t$, diremos que el grafo $\mathcal{G}=(S, \mathcal{V})$ es el grafo asociado a la representación $C A R$ de $Y$. 


\subsubsection{Grafo asociado a modelo SAR}

Sean $X=\left\{X_{s} / s \in S\right\}$ un proceso y $\varepsilon=\left\{\varepsilon_{s} / s \in S\right\}$ un ruido blanco gaussiano con varianza $0<\sigma_{\varepsilon}^{2}:=E\left(\varepsilon_{s}^{2}\right)$ cualquiera sea $s \in S$.

(Equivalentemente: $\varepsilon=\left\{\varepsilon_{s} / s \in S\right\}$ son v.a.i.i.d. con $\varepsilon \sim \mathcal{N}\left(0, \sigma_{\varepsilon}^{2}\right)$, $s \in S$ cualquiera).

Supongamos que $X$ admite representación $A R$ con innovación $\varepsilon$ dada por:

$$
A X^{*}=\varepsilon^{*} \quad ; \quad A=\left[a_{(s, t)}\right]_{(s, t) \in S \times S} .
$$

Supongamos que $A$ es invertible. Luego, $X$ es centrado. También se tiene que $\Sigma\left(X^{*}\right)$ es invertible y

$$
\Sigma\left(X^{*}\right)^{-1}=\sigma_{\varepsilon}^{-2} A^{t} A .
$$

Para cada $t \in S$ sea

$$
\vec{V}_{t}:=\left\{s \in S / a_{(s, t)} \neq 0\right\} .
$$

Notemos que puede suceder:

$$
a_{(s, t)} \neq 0 \text { y } a_{(t, s)}=0 .
$$

Diremos que $\mathcal{G}=\left(S, \overrightarrow{\mathcal{V}}:=\left\{\vec{V}_{t} / t \in S\right\}\right)$ es un grafo orientado llamado grafo orientado asociado a la representación $S A R$ de $X$ dada por (2.3).

Sea:

$$
Q=\Sigma\left(X^{*}\right)^{-1}
$$

y pongamos

$$
Q=\left[q_{(s, t)}\right]_{(s, t) \in S \times S} .
$$

$[Q]$ como en la nota 2.15.11; esto es

$$
\begin{gathered}
{[Q](s, t):=\left\{\begin{array}{cc}
0 & \text { si } s=t \\
q_{(s, t)} & \text { si } s \neq t .
\end{array}\right.} \\
\text { Diag }:=\text { Diagonal }\left(q_{(s, s)} / s \in S\right) .
\end{gathered}
$$

Entonces $X$ tiener una representación $C A R$ con matriz de coeficientes:

$$
C=-\operatorname{Diag}^{-1} \cdot[Q] .
$$

Ejemplo 2.15.12. Representación $C A R$ asociada al modelo $S A R$ local uniparamétrico.

Consideremos la situación de la Definicion 2.15.5 con $\varepsilon=\left\{\varepsilon_{s} / s \in S\right\}$ un ruido blanco gaussiano con varianza $\sigma_{\varepsilon}^{2}>0$. 
Aplicando lo visto en la Nota 2.15.11 con

$$
A(\rho):=I d-\rho W,
$$

y suponiendo $A(\rho)$ invertible, tenemos:

$$
\begin{aligned}
Q(\rho) & :=\Sigma\left(X^{*}\right)^{-1}=\sigma_{\varepsilon}^{-2}(I d-\rho W)^{t}(I d-\rho W) \\
& =\sigma_{\varepsilon}^{-2}\left(I d-\rho\left(W+W^{t}\right)+\rho^{2} W^{t} W\right) .
\end{aligned}
$$

Entonces $X$ tiene una representación CAR con matriz de coeficientes:

$$
C(\rho):=-(\operatorname{Diag}(\rho))^{-1}[Q(\rho)],
$$

siendo:

$$
\begin{gathered}
\operatorname{Diag}(\rho):=\text { Diagonal de } Q(\rho) \\
{[Q(\rho)]_{(s, t)}:=\left\{\begin{array}{cc}
0 & \text { si } s=t \\
Q(\rho)_{(s, t)} & \text { si } s \neq t .
\end{array}\right.}
\end{gathered}
$$

Por la Proposición 2.14.6, si $\rho$ es conocido tenemos que

$$
\hat{X}:=-(\operatorname{Diag}(\rho))^{-1}[Q(\rho)] X
$$

es un predictor lineal óptimo de $X$.

Si $\rho$ no es conocido, en la fórmula anterior se remplazará $\rho$ por un estimador $\hat{\rho}$ definido a partir de los valores de $X$.

\subsection{Modelos de Regresión Espacial}

Sean: $S \subset \mathbb{Z}^{2}$ finito.

Definición 2.16.1. Se dice que $X=\left\{X_{s} / s \in S\right\}$ con $X_{s} \in \mathcal{L}^{2}(\Omega, \mathcal{F}, P, \mathbb{R})$ $\forall s \in S$ satisface un modelo de regresión espacial sobre $S$ si existen $m: S \mapsto \mathbb{R}$ y $\varepsilon=\left\{\varepsilon_{s} / s \in S\right\}$ con $E\left(\varepsilon_{s}\right)=0 \forall s \in S$ tales que

$$
X_{s}=m(s)+\varepsilon_{s} \quad \forall s \in S .
$$

Según cómo sea $m$ se tienen varios ejemplos dentro de este modelo.

Ejemplo 2.16.2. Superficie de respuesta.

Sean $p \geq 1$ entero; $f_{l}: S \mapsto \mathbb{R}, l=1, \ldots p ; \beta_{1}, \ldots, \beta_{p}$ en $\mathbb{R}$. En este caso se define:

$$
m(s)=\sum_{l=1}^{p} \beta_{l} f_{l}(s) .
$$

Desde el punto de vista estadístico se considera que $f_{1}, \ldots, f_{p}$ son funciones conocidas y $\beta_{1}, \ldots, \beta_{p}$ son parámetros a estimar (juntamente con $\Sigma(\varepsilon)$ ). 


\section{Ejemplo 2.16.3. Dependencia exógena.}

Sean: $p \geq 1$ entero; $Z_{l}: \Omega \mapsto \mathbb{R}^{S}$ v.a. para $l=1, \ldots p$.

$\beta_{1}, \ldots, \beta_{p}$ en $\mathbb{R}$. En este caso se define:

$$
m(s)=\sum_{l=1}^{p} \alpha_{l} z_{l}(s),
$$

donde $z_{l}: S \mapsto \mathbb{R}$ es una realización de $Z_{l}$, para todo $l=1, \ldots$.

Desde el punto de vista estadístico $\alpha_{1}, \ldots, \alpha_{p}$ son parámetros a ser estimados y los valores $z_{l}(s) s \in S, l=1, \ldots, p$ se consideran conocidos (valores observados de las variables "exógenas" $Z_{l}$ ).

Ejemplo 2.16.4. Análisis de la varianza.

Supongamos $S=\{(i, j) / 0 \leq i \leq I, 0 \leq j \leq J\}, \mu, \alpha_{1}, \ldots, \alpha_{I}, \beta_{1}, \ldots, \beta_{J}$ en $\mathbb{R}$. Se define

$$
m(i, j)=\mu+\alpha_{i}+\beta_{j} \quad 0 \leq i \leq I, 0 \leq j \leq J .
$$

Desde el punto de vista estadístico $\mu, \alpha_{1}, \ldots, \alpha_{I}, \beta_{1}, \ldots, \beta_{J}$ son parámetros a ser estimados, bajo la condición $\sum_{i=1}^{I} \alpha_{i}=0, \sum_{j=1}^{J} \beta_{j}=0$.

Análisis de la covarianza.

Supongamos $S=\{(i, j) / 0 \leq i \leq I, 0 \leq j \leq J\}$, para cada $(i, j) \in S$ : $Z_{(i, j)}: \Omega \mapsto \mathbb{R}$ v.a., $z_{(i, j)}$ una realización de $Z_{(i, j)}$

$\mu, \alpha_{1}, \ldots, \alpha_{I}, \beta_{1}, \ldots, \beta_{J}, \gamma$ en $\mathbb{R}$. Se define:

$$
\begin{aligned}
& m(i, j)=\mu+\alpha_{i}+\beta_{j}+\gamma z_{(i, j)} \quad 0 \leq i \leq I, 0 \leq j \leq J, \\
& \operatorname{con} \sum_{i=1}^{I} \alpha_{i}=0, \sum_{j=1}^{J} \beta_{j}=0 .
\end{aligned}
$$

Desde el punto de vista estadístico $\mu, \alpha_{1}, \ldots, \alpha_{I}, \beta_{1}, \ldots, \beta_{J}, \gamma$ son parámetros a estimar.

Notación 2.16.5. En el Ejemplo 2.16.3 supongamos que $N=\#(S)$ y sea $\mathbb{I}: S \mapsto\{1, \ldots, N\}$ una biyección .

Sean:

1. $\tilde{X}$ matriz aleatoria $N \times 1$ dada por

$$
\tilde{X}=\left(X_{\mathbb{I}^{-1}(1)}, \ldots, X_{\mathbb{I}^{-1}(N)}\right)^{\prime} ;
$$

2. $\tilde{z}$ matriz $N \times p$ dada por

$$
\tilde{z}=\left[\begin{array}{ccc}
z_{1}\left(\mathbb{I}^{-1}(1)\right) & \cdots & z_{p}\left(\mathbb{I}^{-1}(1)\right) \\
\vdots & \ddots & \vdots \\
z_{1}\left(\mathbb{I}^{-1}(N)\right) & \cdots & z_{p}\left(\mathbb{I}^{-1}(N)\right)
\end{array}\right]
$$


3. $\tilde{\alpha}$ la matriz $p \times 1$ dada por

$$
\tilde{\alpha}=\left(\alpha_{1}, \ldots, \alpha_{p}\right)^{\prime}
$$

4. $\tilde{\varepsilon}$ matriz aleatoria $N \times 1$ dada por

$$
\tilde{\varepsilon}=\left(\varepsilon_{\mathbb{I}^{-1}(1)}, \ldots, \varepsilon_{\mathbb{I}^{-1}(N)}\right)^{\prime} .
$$

Entonces el modelo de dependencia exógena se puede escribir en notación matricial como

$$
\tilde{X}=\tilde{z} \cdot \tilde{\alpha}+\tilde{\varepsilon}
$$

El problema consiste en estimar $\tilde{\alpha}$ y si $\Sigma:=\Sigma(\tilde{\varepsilon})$ es la matriz de covarianza de $\tilde{\varepsilon}$ es desconocida, entonces estimarla modelándola por medio de una función de covarianza $C_{\tilde{\varepsilon}}^{0}$, variograma o modelo $A R$ espacial.

\subsection{Predicción con varianza conocida}

Definición 2.17.1. Sean: $X:=\left\{X_{s} / s \in \mathbb{Z}^{2}\right\}$ con $X_{s} \in \mathcal{L}^{2}(\Omega, \mathcal{F}, P, \mathbb{R})$ centrado; $S=\left\{s_{1}, \ldots, s_{n}\right\} \subset \mathbb{Z}^{2}$ finito $(n \geq 2) ; X_{S}:=\left\{X_{s_{j}} / j=1, \ldots n\right\}$; $\mathcal{L}\left(X_{s}\right):=\left\{a_{1} X_{s_{1}}+\ldots+a_{n} X_{s_{n}} / a_{j} \in \mathbb{R} \forall 1 \leq j \leq n\right\}$. Sea $s_{0} \in \mathbb{Z}^{2}, s_{0} \notin S$, se dice que $\hat{X}_{s_{0}} \in \mathcal{L}\left(X_{s}\right)$ es el predictor lineal óptimo de $X_{s_{0}}$ si

$$
\left\|\hat{X}_{s_{0}}-X_{s_{0}}\right\|_{\mathcal{L}^{2}} \leq\left\|Y-X_{s_{0}}\right\|_{\mathcal{L}^{2}}
$$

cualquiera sea $Y \in \mathcal{L}\left(X_{s}\right)$.

Proposición 2.17.2. Continuación de la Definición 2.17.1. Sean: $\tilde{c}:=\left(c_{1}, \ldots, c_{n}\right)^{\prime} \operatorname{con} c_{j}=\operatorname{Cov}\left(X_{s_{j}}, X_{s_{0}}\right) ; \sigma_{0}^{2}:=\operatorname{Var}\left(X_{s_{0}}\right) ; \Sigma:=\Sigma\left(\tilde{X}_{S}\right)$ con $\tilde{X}_{S}:=\left(X_{s_{1}}, \ldots, X_{s_{n}}\right)^{\prime}$. Sea $\hat{X}_{s_{0}}:=\tilde{c}^{\prime} \Sigma^{-1} \tilde{X}_{S}$, entonces

a) $\hat{X}_{s_{0}}$ es el predictor lineal óptimo de $X_{s_{0}}$.

b) $\operatorname{Var}\left(\hat{X}_{s_{0}}\right)=\tilde{c}^{\prime} \Sigma^{-1} \tilde{c}$.

c) $E\left(\left(\hat{X}_{s_{0}}-X_{s_{0}}\right)^{2}\right)=\sigma_{0}^{2}-\tilde{c}^{\prime} \aleph^{-1} \tilde{c}$.

A $\hat{X}_{s_{0}}$ se lo suele llamar kriging simple para estimar $X_{s_{0}}$ basándose en $\tilde{X}_{S}$, suponiendo conocidas (estimados previamente) $\tilde{\alpha}$ y $\Sigma$. 


\subsubsection{Kriging Universal}

Sean: $X:=\left\{X_{s} / s \in \mathbb{Z}^{2}\right\}$ un proceso en $\mathcal{L}^{2}(\Omega, \mathcal{F}, P, \mathbb{R}) ; p \geq 1$ entero; para cada $l=1, \ldots, p$ sea $Z_{l}:=\left\{Z_{l, s} / s \in \mathbb{Z}^{2}\right\}$ un proceso en $\mathcal{L}^{2}(\Omega, \mathcal{F}, P, \mathbb{R})$.

Supongamos que $\exists \tilde{\alpha} \in \mathbb{R}^{p}$ tal que

$$
X_{s}=\alpha_{1} Z_{1, s}+\ldots+\alpha_{p} Z_{p, s}+\varepsilon_{s}, s \in \mathbb{Z}^{2}
$$

donde $\varepsilon=\left\{\varepsilon_{s} / s \in \mathbb{Z}^{2}\right\}$ es un proceso en $\mathcal{L}^{2}(\Omega, \mathcal{F}, P, \mathbb{R})$ con $E\left(\varepsilon_{s}\right)=0$ $\forall s \in \mathbb{Z}^{2}$.

Sean

$$
\begin{aligned}
\Lambda & :=\left\{s_{1}, \ldots, s_{n}\right\} \subset \mathbb{Z}^{2} \text { con } n \geq 1, \\
s_{0} & \notin \Lambda \\
\tilde{X}_{\Lambda} & =\left(X_{s_{1}}, \ldots, X_{s_{n}}\right)^{\prime}, \\
\widetilde{Z}_{\Lambda} & =\left[\begin{array}{ccc}
Z_{1, s_{1}} & \cdots & Z_{p, s_{1}} \\
\vdots & \ddots & \vdots \\
Z_{1, s_{n}} & \cdots & Z_{p, s_{n}}
\end{array}\right], \\
\widetilde{Z}_{s_{0}} & =\left(Z_{1, s_{0}}, \ldots, Z_{p, s_{0}}\right)^{\prime}, \\
\mathcal{L}\left(\tilde{X}_{\Lambda}\right) & =\left\{\tilde{a}^{\prime} \tilde{X}_{\Lambda} / \tilde{a} \in \mathbb{R}^{n}\right\} . \\
\mathcal{L}\left(\tilde{X}_{\Lambda}, X_{s_{0}}, \widetilde{Z}_{\Lambda}, \widetilde{Z}_{s_{0}}\right) & =\left\{Y \in \mathcal{L}\left(\tilde{X}_{\Lambda}\right) / E\left(Y \mid \widetilde{Z}_{\Lambda}\right)=E\left(X_{s_{0}} \mid \widetilde{Z}_{s_{0}}\right)\right\} .
\end{aligned}
$$

Se dice que $\hat{X}_{s_{0}} \in \mathcal{L}\left(\tilde{X}_{\Lambda}, X_{s_{0}}, \widetilde{Z}_{\Lambda}, \widetilde{Z}_{s_{0}}\right)$ es un estimador linear insesgado óptimo de $X_{s_{0}}$ conocidos $\tilde{Z}_{\Lambda}$ y $\tilde{Z}_{s_{0}}$ si

$$
\left\|\hat{X}_{s_{0}}-X_{s_{0}}\right\|_{\mathcal{L}^{2}} \leq\left\|Y-X_{s_{0}}\right\|_{\mathcal{L}^{2}}
$$

cualquiera sea $Y \in \mathcal{L}\left(\tilde{X}_{\Lambda}, X_{s_{0}}, \widetilde{Z}_{\Lambda}, \widetilde{Z}_{s_{0}}\right)$.

Proposición 2.17.3. Sean:

$\tilde{c}:=\left(c_{1}, \ldots, c_{n}\right)^{\prime} \operatorname{con} c_{j}=\operatorname{Cov}\left(X_{s_{j}}, X_{s_{0}}\right) ; \sigma_{0}^{2}:=\operatorname{Var}\left(X_{s_{0}}\right) ; \Sigma:=\Sigma\left(\tilde{X}_{\Lambda}\right)$. Sea $\hat{X}_{s_{0}}=\tilde{\lambda}^{\prime} \tilde{X}_{\Lambda}$ con $\tilde{\lambda}:=\Sigma^{-1} \widetilde{Z}_{\Lambda}\left(\widetilde{Z}_{\Lambda}^{\prime} \Sigma^{-1} \widetilde{Z}_{\Lambda}\right)^{-1}\left(\widetilde{Z}_{s_{0}}-\widetilde{Z}_{\Lambda}^{\prime} \aleph^{-1} \tilde{c}\right)+\Sigma^{-1} \tilde{c}$. Entonces:

a) $\hat{X}_{s_{0}}$ es el estimador lineal insesgado óptimo de $X_{s_{0}}$ conocidos $\widetilde{Z}_{\Lambda}$ y $\widetilde{Z}_{s_{0}}$. b) $\operatorname{Var}\left(\hat{X}_{s_{0}}\right)=\sigma_{0}^{2}-\tilde{c}^{\prime} \Sigma^{-1} \tilde{c}+\left(\widetilde{Z}_{s_{0}}-\widetilde{Z}_{\Lambda}^{\prime} \Sigma^{-1} \tilde{c}\right)^{\prime}\left(\widetilde{Z}_{\Lambda}^{\prime} \Sigma^{-1} \widetilde{Z}_{\Lambda}\right)^{-1}\left(\widetilde{Z}_{s_{0}}-\right.$
$\left.\widetilde{Z}_{\Lambda}^{\prime} \sharp^{-1} \tilde{c}\right)$. 


\section{Capítulo 3}

\section{Campos Aleatorios de Gibbs Markov sobre Redes}

Sean:

- $E$ un espacio métrico separable y completo;

- $\mathcal{E}$ la $\sigma$-algebra de Borel de $E$.

- $S \subset \mathbb{Z}^{2}$ a lo sumo numerable;

- $\mathcal{S}:=\{\Lambda \subset S / 1 \leq \#(\Lambda)<\infty\}$.

- Para cada $\emptyset \neq V \subset S$ sea

$$
E^{V}:=\{x / x: V \longmapsto E\} .
$$

Sean $\emptyset \neq V_{1} \subset V_{2} \subset S$, definimos $\sigma_{V_{2}, V_{1}}: E^{V_{2}} \longmapsto E^{V_{1}}$ por:

$$
\sigma_{V_{2}, V_{1}}(x)(t)=x(t), \quad t \in V_{1}, x \in E^{V_{2}} .
$$

- Si $V_{2}=S$ ponemos $\sigma_{V_{1}}$ en lugar $\sigma_{S, V_{1}}$.

- Sea $\emptyset \neq V \subset S$. Definimos

$$
G_{V}:=\left\{\sigma_{V, s}^{-1}(B) / B \in \mathcal{E}, s \in V\right\} .
$$

- Sea $\mathcal{E}^{V}$ la $\sigma$-álgebra de $E^{V}$ generada por $G_{V}$. A esta $\sigma$-álgebra se la suele llamar $\sigma$-álgebra producto de $E^{V}$ inducida por $\mathcal{E}$. 
- Sea $\mathcal{F}_{V}$ la $\sigma$-álgebra de $E^{S}$ definida por:

$$
\mathcal{F}_{V}:=\left\{\sigma_{V}^{-1}(B) / B \in \mathcal{E}^{V}\right\} .
$$

- Por simplicidad pondremos

$$
\mathcal{F}:=\mathcal{E}^{S}
$$

- También por simplicidad pondremos

$$
\mathcal{J}_{\Lambda}:=\mathcal{F}_{S \backslash V} \quad \Lambda \in \mathcal{S} .
$$

Lema 3.0.4 (útil). Sea $\emptyset \neq V \varsubsetneqq S . f: E^{S} \longmapsto \mathbb{R} \mathcal{F}$-medible. Entonces $f$ es $\mathcal{F}_{V}$-medible si y sólo si es cierta

$$
\left(x \in E^{S}, y \in E^{S}, \sigma_{V}(x)=\sigma_{V}(y)\right) \Rightarrow f(x)=f(y) .
$$

Demostración. Ejercicio (Ayuda: ver Theorem B, pag. 142 de Halmos $(1974) \cdot([8]))$

\subsection{Potenciales y distribuciones de Gibbs}

Definición 3.1.1. Para cada $s \in S$ sea $\Phi:=\left\{\Phi_{\Lambda} / \Lambda \in \mathcal{S}\right\}$. $\Phi$ es un potencial sobre $\left(E^{S}, \mathcal{F}\right)$ si:

i) Para cada $\Lambda \in \mathcal{S}, \Phi_{\Lambda}$ es $\mathcal{F}_{\Lambda}$-medible;

ii) Para cada $\Lambda \in \mathcal{S}$ y cada $x \in E^{S}$, existe en $\mathbb{R}$

$$
H_{\Lambda}^{\Phi}(x):=\sum_{\Delta \in \mathcal{S} \cap \Lambda} \Phi_{\Delta}(x),
$$

donde $\mathcal{S} \cap \Lambda:=\{\Delta \in \mathcal{S} / \Delta \cap \Lambda \neq \emptyset\}$.

A la función $H_{\Lambda}^{\Phi}$ se la llama energía del potencial $\Phi$ sobre $\Lambda$.

Pondremos: $H^{\Phi}:=\left\{H_{\Lambda}^{\Phi} / \Lambda \in \mathcal{S}\right\}$.

Definición 3.1.2. Sean: $\lambda$ una medida $\sigma$-finita no nula sobre $(E, \mathcal{E}) ; \Phi=$ $\left\{\Phi_{\Lambda} / \Lambda \in \mathcal{S}\right\}$ un potencial sobre $\left(E^{S}, \mathcal{F}\right)$. Se dice que $\Phi$ es $\lambda$-admisible si

$$
0<\int_{E^{\Lambda}} \exp \left(-H_{\Lambda}^{\Phi}\left(\xi x_{S \backslash \Lambda}\right)\right) \lambda^{\Lambda}(d \xi)<\infty,
$$

para todo $x \in E^{S}$ y $\Lambda \in \mathcal{S}$, donde $\lambda^{\Lambda}$ es la única medida sobre $\left(E^{\Lambda}, \mathcal{E}^{\Lambda}\right)$ tal que si $\Lambda=\left\{s_{1}, \ldots, s_{n}\right\}$, entonces:

$$
\begin{gathered}
\lambda^{\Lambda}\left(\sigma_{\Lambda,\left\{s_{1}\right\}}^{-1}\left(B_{1}\right) \cap \ldots \cap \sigma_{\Lambda,\left\{s_{n}\right\}}^{-1}\left(B_{n}\right)\right)= \\
\lambda\left(B_{1}\right) \ldots \lambda\left(B_{n}\right)
\end{gathered}
$$

cualesquiera sean $B_{1}, \ldots, B_{n}$ en $\mathcal{E}$. 
Para simplificar la notación, pondremos

$$
h_{\Lambda}^{\Phi}(x):=\exp \left(-H_{\Lambda}^{\Phi}(x)\right)
$$

para todo $x \in E^{S}$ y $\Lambda \in \mathcal{S}$.

En el caso en que $\Phi$ es $\lambda$-admisible, definimos

$$
Z_{\Lambda, \lambda}^{\Phi}(x):=\int_{E^{\Lambda}} \exp \left(-H_{\Lambda}^{\Phi}\left(\xi x_{S \backslash \Lambda}\right)\right) \lambda^{\Lambda}(d \xi), x \in E^{S} .
$$

A esta función $Z_{\Lambda, \lambda}^{\Phi}$ la llamamos función partición del potencial $\Phi$ sobre $\Lambda$.

Pondremos: $Z_{\lambda}^{\Phi}:=\left\{Z_{\Lambda, \lambda}^{\Phi} / \Lambda \in \mathcal{S}\right\}$.

Proposición 3.1.3. Sean $\lambda$ una medida $\sigma$-finita sobre $(E, \mathcal{E}) ; \Phi=\left\{\Phi_{\Lambda}\right.$ : $\Lambda \in \mathcal{S}\}$ un potencial sobre $\left(E^{S}, \mathcal{F}\right) \lambda$-admisible.

Para cada $\Lambda \in \mathcal{S}$ sea $\varrho_{\Lambda, \lambda}^{\Phi}: E^{S} \longmapsto[0,+\infty)$ dada por:

$$
\varrho_{\Lambda, \lambda}^{\Phi}(x):=\frac{h_{\Lambda}^{\Phi}(x)}{Z_{\Lambda, \lambda}^{\Phi}(x)}, x \in E^{S} .
$$

Para cada $\Lambda \in \mathcal{S}$ sea $\gamma_{\Lambda, \lambda}^{\Phi}: \mathcal{F} \times E^{S} \longmapsto[0,1]$ dada por

$$
\gamma_{\Lambda, \lambda}^{\Phi}(A \mid x):=\int_{E^{\Lambda}} 1_{A}\left(\xi x_{S \backslash \Lambda}\right) \varrho_{\Lambda, \lambda}^{\Phi}\left(\xi x_{S \backslash \Lambda}\right) \lambda^{\Lambda}(d \xi),
$$

para todo $A \in \mathcal{F}$ y $x \in E^{S}$.

Entonces:

a) Para cada $A \in \mathcal{F}, \gamma_{\Lambda, \lambda}^{\Phi}(A \mid \cdot)$ es $\mathcal{J}_{\Lambda}$-medible

b) Para cada $x \in E^{S}, \gamma_{\Lambda, \lambda}^{\Phi}(A \mid \cdot) \in \mathcal{P}\left(E^{S}, \mathcal{F}\right)$ (es una probabilidad sobre $\left.\left(E^{S}, \mathcal{F}\right)\right)$.

c) Si $\Lambda \subset \Delta$, ambos en $\mathcal{S}$, entonces

$$
\int_{E^{S}} \gamma_{\Lambda, \lambda}^{\Phi}(A \mid \omega) \gamma_{\Delta, \lambda}^{\Phi}(d \omega \mid x)=\gamma_{\Delta, \lambda}^{\Phi}(A \mid x)
$$

cualquiera sean $A \in \mathcal{F}, x \in E^{S}$.

d) $B \in \mathcal{J}_{\Lambda} \Longrightarrow \gamma_{\Lambda, \lambda}^{\Phi}(B \mid x)=1_{B}(x) \forall x \in E^{S}$.

e) $A \in \mathcal{F}, B \in \mathcal{J}_{\Lambda} \Longrightarrow \gamma_{\Lambda, \lambda}^{\Phi}(A \cap B \mid x)=\gamma_{\Lambda, \lambda}^{\Phi}(A \mid x) 1_{B}(x)$.

Demostración. Ejercicio.

Definición 3.1.4. En la situación de la proposición anterior a $\gamma_{\lambda}^{\Phi}:=$ $\left(\gamma_{\Lambda, \lambda}^{\Phi}\right)_{\Lambda \in \mathcal{S}}$ se la llama especificación inducida por $\Phi \boldsymbol{y} \lambda$. 
Definición 3.1.5. Sean: $\lambda$ una medida $\sigma$-finita sobre $(E, \mathcal{E}) ; \Phi=$ $\left\{\Phi_{\Lambda}: \Lambda \in \mathcal{S}\right\}$ un potencial sobre $\left(E^{S}, \mathcal{F}\right) \lambda$-admisible; $\gamma_{\lambda}^{\Phi}:=\left(\gamma_{\Lambda, \lambda}^{\Phi}\right)_{\Lambda \in \mathcal{S}}$ la especificación inducida por $\Phi$ y $\lambda$.

Pondremos:

$$
\mathcal{G}\left(\gamma_{\lambda}^{\Phi}\right):=\left\{\mu \in \mathcal{P}\left(E^{S}, \mathcal{F}\right) / \mu \gamma_{\Lambda, \lambda}^{\Phi}=\mu \forall \Lambda \in \mathcal{S}\right\}
$$

Esto es: $\mu \in \mathcal{G}\left(\gamma_{\lambda}^{\Phi}\right)$ si y sólo si para cada $\Lambda \in \mathcal{S}$ y cada $A \in \mathcal{F}$, $\gamma_{\Lambda, \lambda}^{\Phi}(A \mid \cdot)$ es una probabilidad condicional de $A$ dada $\mathcal{J}_{\Lambda}$ con respecto a $\mu$ ( en símbolos: $\gamma_{\Lambda}^{\Phi}(A \mid \cdot) \in \mu\left(A \mid \mathcal{J}_{\Lambda}\right)$ ).

Notación 3.1.6. Si $f: E^{S} \rightarrow \mathbb{R}$ es $\mathcal{F}$-medible, siempre que tenga sentido ponemos

$$
\gamma_{\Lambda}^{\Phi}(f)(x):=\int_{E^{S}} f(\omega) \gamma_{\Lambda}^{\Phi}(d \omega \mid x)
$$
a $\gamma_{\lambda}^{\Phi}$.

Si $\mu \in \mathcal{G}\left(\gamma_{\lambda}^{\Phi}\right)$ diremos que $\mu$ es una distribución de Gibbs asociada Nota 3.1.7. $\mathcal{G}\left(\gamma_{\lambda}^{\Phi}\right)$ puede ser vacía, o tener un solo elemento o tener infinitos elementos (transición de fase).

A continuación veremos una clase de potencial para el que $\mathcal{G}\left(\gamma_{\lambda}^{\Phi}\right) \neq \emptyset$ en condiciones bastante generales.

Definición 3.1.8. Sea $\mathcal{V}:=\left\{V_{s}: s \in S\right\}$ una subfamilia (no vacía) de $\mathcal{S}$. Se dice que $\mathcal{V}$ es un sistema de vecindades de $S$ si:

i) $s \notin V_{s}, \forall s \in S$.

ii) $s \in V_{t} \Leftrightarrow t \in V_{s} ; s$ y $t$ en $S s \neq t$.

iii) $S=\bigcup_{s \in S} V_{s}$.

En este caso, para cada $\Lambda \in \mathcal{S}$ definimos

$$
\partial_{\mathcal{V}}(\Lambda):=\left\{t \notin \Lambda / \exists s \in \Lambda \text { con } t \in V_{s}\right\} .
$$

Definición 3.1.9. Sean: $\lambda$ una medida $\sigma$-finita sobre $(E, \mathcal{E}) ; \Phi=$ $\left\{\Phi_{\Lambda} / \Lambda \in \mathcal{S}\right\}$ un potencial sobre $\left(E^{S}, \mathcal{F}\right) \lambda$-admisible; $\gamma_{\lambda}^{\Phi}=\left(\gamma_{\Lambda, \lambda}^{\Phi}\right)_{\Lambda \in \mathcal{S}}$ la especificación inducida por $\Phi$ y $\lambda ; \mathcal{V}:=\left\{V_{s} / s \in S\right\}$ un sistema de vecindades de $S ; \mathcal{G}:=(S, \mathcal{V})$ el grafo sobre $S$ inducido por $\mathcal{V}$. Se dice que $\gamma_{\lambda}^{\Phi}$ es $\mathcal{G}$-markoviana si $\gamma_{\Lambda, \lambda}^{\Phi}(A \mid \cdot)$ es $\mathcal{F}_{\partial_{\mathcal{V}}(\Lambda)}$-medible, $\forall A \in \mathcal{F}_{\Lambda}, \forall \Lambda \in \mathcal{S}$.

Definición 3.1.10. Sea $\Phi=\left\{\Phi_{\Lambda} / \Lambda \in \mathcal{S}\right\}$ un potencial sobre $\left(E^{S}, \mathcal{F}\right)$. Se dice que $\Phi$ es acotado si $\Phi_{\Lambda} \in \mathcal{L}^{\infty}\left(E^{S}, \mathcal{F}, \mathbb{R}\right), \forall \Lambda \in \mathcal{S}$. 
Definición 3.1.11. Sea $\Phi=\left\{\Phi_{\Lambda} / \Lambda \in \mathcal{S}\right\}$ un potencial sobre $\left(E^{S}, \mathcal{F}\right)$. Si $\Phi$ es acotado se dice que $\Phi$ es sumable si

$$
\sum_{\Lambda \in \mathcal{S} \cap\{s\}}\left\|\Phi_{\Lambda}\right\|_{\mathcal{L}^{\infty}}<\infty, \forall s \in S .
$$

En este caso, pondremos:

$$
\left\|\Phi_{\Lambda}\right\|_{s}:=\sum_{\Lambda \in \mathcal{S} \cap\{s\}}\left\|\Phi_{\Lambda}\right\|_{\mathcal{L}^{\infty}}, \forall s \in S .
$$

Proposición 3.1.12. $\Phi=\left\{\Phi_{\Lambda} / \Lambda \in \mathcal{S}\right\}$ un potencial sobre $\left(E^{S}, \mathcal{F}\right), \lambda$ una medida $\sigma$-finita sobre $(E, \mathcal{E})$.

Si $\Phi$ es sumable, entonces, $\Phi$ es $\lambda$-admisible $\Leftrightarrow \lambda(E)<\infty$.

Demostración. $\Leftarrow\rfloor$ Sean $\Lambda \in \mathcal{S}, x \in E^{S}$ cualesquiera. Entonces:

$$
\begin{gathered}
\exp \left(-\sum_{\Delta \in \mathcal{S} \cap \Lambda} \Phi_{\Delta}\left(\xi x_{S \backslash \Lambda}\right)\right) \leq \exp \left(\left|\sum_{\Delta \in \mathcal{S} \cap \Lambda} \Phi_{\Delta}\left(\xi x_{S \backslash \Lambda}\right)\right|\right) \\
\leq \exp \left(\sum_{\Delta \in \mathcal{S} \cap \Lambda}\left\|\Phi_{\Delta}\right\|_{s}\right) \quad \forall \xi \in E^{\Lambda} .
\end{gathered}
$$

Luego:

$$
\begin{aligned}
\int_{E^{\Lambda}} \exp \left(-H_{\Lambda}^{\Phi}\left(\xi x_{S \backslash \Lambda}\right)\right) \lambda^{\Lambda}(d \xi) \leq \exp \left(\sum_{\Delta \in \mathcal{S} \cap \Lambda}\left\|\Phi_{\Delta}\right\|_{s}\right)(\lambda(E))^{\#(\Lambda)}<\infty \\
\Rightarrow\rfloor \text { Sean } s \in S, x \in E^{S} \text { cualesquiera. Entonces: } \\
\sum_{\Delta \in \mathcal{S} \cap\{s\}} \Phi_{\Delta}\left(\xi x_{S \backslash s\}}\right) \leq\|\Phi\|_{s}<\infty \quad \forall \xi \in E .
\end{aligned}
$$

Luego:

$$
\begin{aligned}
\infty & >\int_{E} \exp \left(-\sum_{\Delta \in \mathcal{S} \cap\{s\}} \Phi_{\Delta}\left(\xi x_{S \backslash\{s\}}\right)\right) \lambda(d \xi) \geq \lambda(E) e^{\|\Phi\|_{s}} \\
\Rightarrow \lambda(E) & <\infty .
\end{aligned}
$$

Nota 3.1.13. Se puede ver, por ejemplo en Theorem (4.23) de Georgii (1988) ([6]), pag. 72 que: $\Phi=\left\{\Phi_{\Lambda} / \Lambda \in \mathcal{S}\right\}$ un potencial sobre $\left(E^{S}, \mathcal{F}\right), \lambda$ una medida $\sigma$-finita sobre $(E, \mathcal{E}), E$ un espacio métrico separable y completo, $\mathcal{E}$ su $\sigma$-álgebra de Borel.

Si $\Phi$ es sumable y $\lambda(E)<\infty$, entonces $\mathcal{G}\left(\gamma_{\lambda}^{\Phi}\right) \neq \emptyset$. 
Es importante entonces estudiar ejemplos de potenciales sumables.

Definición 3.1.14. Sean : $\mathcal{V}$ un sistema de vecindades de $S, \Phi=\left(\Phi_{\Lambda}\right)_{\Lambda \in \mathcal{S}}$ un potencial sobre $\left(E^{S}, \mathcal{F}\right)$. Se dice que $\Phi$ es un $\mathcal{G}=(S, V)$-potencial si

$$
\Phi_{\Lambda}=0 \quad \forall \Lambda \notin \mathcal{C}(\mathcal{G})
$$

donde

$$
\mathcal{C}(\mathcal{G}):=\left\{C \in S / \#(C)=1 \text { os } s \in C, t \in C, s \neq t \Rightarrow t \in \mathcal{V}_{s}\right\} .
$$

A cada elemento de $\mathcal{C}(\mathcal{G})$ lo llamaremos $\mathcal{G}$-completo.

Proposición 3.1.15. Sean: $\mathcal{V}$ un sistema de vecindades de $S, \Phi=\left(\Phi_{\Lambda}\right)_{\Lambda \in \mathcal{S}}$ un potencial sobre $\left(E^{S}, \mathcal{F}\right), \lambda$ una medida $\sigma$-finita sobre $(E, \mathcal{E})$. Si $\Phi$ es un $\mathcal{G}=(S, \mathcal{V})$-potencial acotado y $\lambda(E)<\infty$, entonces $\Phi$ es sumable.

Luego, $\Phi$ es admisible y $\mathcal{G}\left(\gamma_{\lambda}^{\Phi}\right) \neq \emptyset$.

Demostración. Sea $\Lambda \in \mathcal{S}$ cualquiera,

Afirmación 1: Si $\Delta \in \mathcal{S} \cap \Lambda$ y $\Delta \in \mathcal{C}(\mathcal{G})$ entonces $\Delta \subset\left(\Lambda \cup \partial_{\mathcal{V}}(\Lambda)\right)$

Demostración. Ejercicio. finita.

Luego, cualquiera sea $\Lambda \in \mathcal{S},\{\Delta \in \mathcal{S} / \Delta \in \mathcal{S} \cap \Lambda$ y $\Delta \in \mathcal{C}(\mathcal{G})\}$ es

Como $\Phi$ es acotado:

$$
\sum_{\Delta \in \mathcal{S} \cap \Lambda}\left\|\Phi_{\Delta}\right\|_{\mathcal{L}^{\infty}}<\infty
$$

En particular $\sum_{\Delta \in \mathcal{S} \cap\{s\}}\left\|\Phi_{\Delta}\right\|_{\mathcal{L}^{\infty}}<\infty, \forall s \in S$

Corolario 3.1.16. Sean: E finito; $\lambda$ la medida de conteo sobre $(E, \mathcal{P}(E))$; $\Phi=\left(\Phi_{\Lambda}\right)_{\Lambda \in \mathcal{S}}$ un potencial sobre $\left(E^{S}, \mathcal{F}\right) ; \mathcal{V}$ un sistema de vecindades de S. Si $\Phi$ es un $\mathcal{G}$-potencial (con $\mathcal{G}=(S, \mathcal{V}))$ entonces $\Phi$ es sumable y por lo tanto $\mathcal{G}\left(\gamma_{\lambda}^{\Phi}\right) \neq \emptyset$.

Demostración. Por la Proposición anterior, bastará ver que $\Phi$ es acotado, esto es, que $\Phi_{\Lambda} \in \mathcal{L}^{\infty}\left(E^{S}, \mathcal{F}, \mathbb{R}\right), \forall \Lambda \in \mathcal{S}$.

Sea $x \in E^{S}$ fijo cualquiera, como $\Phi_{\Lambda}$ es $\mathcal{F}_{\Lambda}$-medible,

$$
\Phi_{\Lambda}(y)=\Phi_{\Lambda}\left(y_{\Lambda} x_{S \backslash \Lambda}\right) \quad \forall y \in E .
$$

Como $E$ es finito y $\Lambda$ también lo es:

$$
\#\left(\left\{\Phi_{\Lambda}\left(\xi x_{S \backslash \Lambda}\right) / \xi \in E^{\Lambda}\right\}\right)<\infty .
$$

Luego por (1):

$$
\left\|\Phi_{\Lambda}\right\|_{\mathcal{L}^{\infty}}=\sup _{y \in E^{S}}\left|\Phi_{\Lambda}(y)\right|<\infty .
$$


Un resultado de interés es el siguiente.

Proposición 3.1.17. Sean: $\mathcal{V}$ un sistema de vecindades de $S, \Phi=\left(\Phi_{\Lambda}\right)_{\Lambda \in \mathcal{S}}$ un potencial sobre $\left(E^{S}, \mathcal{F}\right), \lambda$ una medida $\sigma$-finita sobre $(E, \mathcal{E})$. Si $\Phi$ es un $\mathcal{G}=(S, \mathcal{V})$-potencial $\lambda$-admisible, entonces $\gamma_{\lambda}^{\Phi}$ es $\mathcal{G}$-markoviana.

Demostración. Sean: $\Lambda \in \mathcal{S}, A \in \mathcal{F}_{\Lambda}$. Debemos probar que $\gamma_{\Lambda, \lambda}^{\Phi}(A \mid \cdot)$ es $\mathcal{F}_{\partial \mathcal{V}(\Lambda)}$-medible.

Como $\gamma_{\Lambda, \lambda}^{\Phi}(A \mid \cdot)$ es $\mathcal{F}$-medible, por Lema útil, bastará ver que:

$x \in E^{S}, y \in E^{S}, \sigma_{\partial \mathcal{V}(\Lambda)}(x)=\sigma_{\partial \mathcal{V}(\Lambda)}(y) \Rightarrow \gamma_{\Lambda, \lambda}^{\Phi}(A \mid x)=\gamma_{\Lambda, \lambda}^{\Phi}(A \mid y)$.

Entonces, sean $x \in E^{S}, y \in E^{S}$ tales que $\sigma_{\partial_{\mathcal{V}}(\Lambda)}(x)=\sigma_{\partial_{\mathcal{V}}(\Lambda)}(y)$.

Afirmación 1: Si $\Delta \in \mathcal{S} \cap \Lambda \Rightarrow \Phi_{\Delta}\left(\xi x_{S \backslash \Lambda}\right)=\Phi_{\Delta}\left(\xi y_{S \backslash \Lambda}\right) \forall \xi \in E^{\Lambda}$.

Supongamos cierta esta afirmación. Como $A \in \mathcal{F}_{\Lambda}, \exists B \in \mathcal{E}^{\Lambda}$ tal que $A=\sigma_{\Lambda}^{-1}(B)$.

Luego:

$$
1_{A}\left(\xi x_{S \backslash \Lambda}\right)=1_{A}\left(\xi y_{S \backslash \Lambda}\right) \quad \forall \xi \in E^{\Lambda} .
$$

Por la Afirmación 1:

$$
H_{\Lambda}^{\Phi}\left(\xi x_{S \backslash \Lambda}\right)=H_{\Lambda}^{\Phi}\left(\xi y_{S \backslash \Lambda}\right) \quad \forall \xi \in E^{\Lambda} .
$$

Luego:

$$
Z_{\Lambda, \lambda}^{\Phi}(x)=Z_{\Lambda, \lambda}^{\Phi}(y) .
$$

Por (3) y (4) tenemos entonces:

$$
\varrho_{\Lambda, \lambda}^{\Phi}\left(\xi x_{S \backslash \Lambda}\right)=\varrho_{\Lambda, \lambda}^{\Phi}\left(\xi y_{S \backslash \Lambda}\right) \quad \forall \xi \in E^{\Lambda} .
$$

De aquí y de (2) por definición de $\gamma_{\Lambda, \lambda}^{\Phi}$ se tiene:

$$
\gamma_{\Lambda, \lambda}^{\Phi}(A \mid x)=\gamma_{\Lambda, \lambda}^{\Phi}(A \mid y) .
$$

Veamos la prueba de la Afirmación 1.

Si $\Phi_{\Delta}=0$ no hay nada que probar.

Supongamos, entonces que $\Phi_{\Delta} \neq 0 \Rightarrow \Delta \in \mathcal{C}(\mathcal{G})$.

Por la afirmación de la proposición anterior se tiene que

$$
\Delta \subset\left(\Lambda \cup \partial_{\mathcal{V}}(\Lambda)\right)
$$

Como $\Phi_{\Lambda}$ es $\mathcal{F}_{\Lambda}$-medible y $\sigma_{\partial_{\mathcal{V}}(\Lambda)}(x)=\sigma_{\partial_{\mathcal{V}}(\Lambda)}(y)$, se tiene que

$$
\Phi_{\Delta}\left(\xi x_{S \backslash \Lambda}\right)=\Phi_{\Delta}\left(\xi y_{S \backslash \Lambda}\right) \forall \xi \in E^{\Lambda} .
$$




\subsection{Ejemplos de Potenciales y distribuciones de Gibbs}

En esta Sección usaremos la siguiente notación.

Notación 3.2.1. Sean: $d=1$ o $d=2$. $S=\mathbb{Z}^{d}$.

a) Si s y $t$ están en $\mathbb{Z}$, pondremos.

$$
\|s-t\|_{1}:=|s-t| .
$$

Si $s=\left(s_{1}, s_{2}\right)$ y $t=\left(t_{1}, t_{2}\right)$ están en $\mathbb{Z} \times \mathbb{Z}$ pondremos,

$$
\|s-t\|_{1}:=\left|s_{1}-t_{1}\right|+\left|s_{2}-t_{2}\right| .
$$

b) Para cada $\delta \in \mathbb{N}$ y $s \in S$ sea

$$
\mathcal{V}_{s}^{\delta}:=\left\{t \in S \backslash\{s\} /\|s-t\|_{1} \leq \delta\right\} .
$$

Pondremos:

$$
\mathcal{V}^{\delta}:=\left\{\mathcal{V}_{s}^{\delta} / s \in S\right\}
$$

Proposición 3.2.2. $\mathcal{V}^{\delta}$ es un sistema de vecindades sobre $S$. Pondremos $\mathcal{G}^{\delta}:=\left(S, \mathcal{V}^{\delta}\right)$ el grafo sobre $S$ inducido por $V^{\delta}$.

Demostración. Ejercicio.

Ejemplo 3.2.3 (Modelo de Ising). Sean: $E:=\{-1,+1\} .(d=1$ o 2$)$.

a) Modelo de Ising sin campo externo.

$$
\begin{aligned}
\text { Sea } \beta \in \mathbb{R} ; \Phi^{\beta}=\left(\Phi_{\Lambda}^{\beta}\right)_{\Lambda \in \mathcal{S}} \text { con } \\
\Phi_{\Lambda}^{\beta}(x)=\left\{\begin{array}{l}
\beta x(s) x(t) \quad \text { si }\{s, t\}=\Lambda \subset \mathcal{V}_{s}^{1} \cup\{s\} s \neq t . \\
0 \quad \text { c.c. }
\end{array}\right.
\end{aligned}
$$

b) Modelo de Ising con campo externo.

$$
\begin{aligned}
\text { Sea } \alpha, \beta & \in \mathbb{R} ; \Phi^{\beta}=\left(\Phi_{\Lambda}^{(\alpha, \beta)}\right)_{\Lambda \in \mathcal{S}} \text { con } \\
\Phi_{\Lambda}^{(\alpha, \beta)}(x) & =\left\{\begin{array}{l}
\alpha x(s) \text { si }\{s\}=\Lambda \\
\beta x(s) x(t) \text { si }\{s, t\}=\Lambda \subset \mathcal{V}_{s}^{1} \cup\{s\} s \neq t . \\
0 \text { c.c. }
\end{array}\right.
\end{aligned}
$$

Afirmación: $\Phi_{\Lambda}^{\beta} y \Phi_{\Lambda}^{(\alpha, \beta)}$ son $\mathcal{G}^{\delta}$-potenciales $(\delta \geq 1)$. Luego, por Corolario 3.1.16 si $\lambda$ es la medida de conteo sobre $(E, \mathcal{P}(E))$ tenemos que

$$
\mathcal{G}\left(\gamma_{\lambda}^{\Phi^{\beta}}\right) \neq \emptyset \quad y \quad \mathcal{G}\left(\gamma_{\lambda}^{\Phi^{(\alpha, \beta)}}\right) \neq \emptyset
$$


Ejemplo 3.2.4 (Modelo de Ising con espacio de estados $\mathrm{E}=\{0,1\}$ o modelo de "presencia-ausencia"). Sean: $E:=\{0,1\} a, b \in \mathbb{R} .(d=1$ o 2$)$.

Sea $\Phi^{(a, b)}:=\left(\Phi_{\Lambda}^{(a, b)}\right)_{\Lambda \in \mathcal{S}}$ con $\Phi_{\Lambda}^{(a, b)}$ definido como $\Phi_{\Lambda}^{(\alpha, \beta)}$ del ejemplo anterior.

Nota 3.2.5 (Algunas precisiones en el caso $S$ finito). Supongamos $S$ finito $(E, \mathcal{E}) ;\left(E^{S}, \mathcal{F}\right)$ como al inicio de esta Sección.

Luego:

$$
\begin{aligned}
H_{S}^{\Phi}(x) & =\sum_{\Delta \in \mathcal{S}} \Phi_{\Delta}(x), \\
h_{S}^{\Phi}(x) & =\exp \left(-H_{S}^{\Phi}(x)\right) .
\end{aligned}
$$

Sea $\lambda$ una medida $\sigma$-finita sobre $(E, \mathcal{E})$. Supongamos que $\Phi$ es $\lambda$-admisible. En este caso $Z_{S, \lambda}^{\Phi}$ es la constante dada por

$$
Z_{S, \lambda}^{\Phi}=\int_{E^{S}} \exp \left(-H_{\Lambda}^{\Phi}(\xi)\right) \lambda^{S}(d \xi)
$$

$\varrho_{S, \lambda}^{\Phi}(x): E^{S} \longmapsto[0,+\infty)$ dada por:

$$
\varrho_{S, \lambda}^{\Phi}(x):=\frac{h_{S}^{\Phi}(x)}{Z_{S, \lambda}^{\Phi}} .
$$

$\gamma_{S, \lambda}^{\Phi}$ será la probabilidad sobre $\left(E^{S}, \mathcal{F}\right)$ con densidad (con respecto a la medida $\left.\lambda^{S}\right) \varrho_{S, \lambda}^{\Phi}$. Esto es

$$
\gamma_{S, \lambda}^{\Phi}(A)=\int_{A} \varrho_{S, \lambda}^{\Phi}(x) \lambda^{S}(d x) \quad A \in \mathcal{F}
$$

Por la Proposición 3.1.3 tenemos que:

$$
\Lambda \in \mathcal{S} \operatorname{con} \Lambda \neq S \Rightarrow \gamma_{\Lambda, \lambda}^{\Phi}(A \mid \cdot) \in \gamma_{S, \lambda}^{\Phi}\left(A \mid \mathcal{J}_{\Lambda}\right), \quad A \in \mathcal{F},
$$

(donde $\mathcal{J}_{\Lambda}=\mathcal{F}_{S \backslash \Lambda}$ por definición).

Luego $\gamma_{S, \lambda}^{\Phi} \in \mathcal{G}\left(\gamma_{\lambda}^{\Phi}\right)$.

Por otra parte, si $\mu \in \mathcal{G}\left(\gamma_{\lambda}^{\Phi}\right)$ por definición debe ser

$$
\int \gamma_{S, \lambda}^{\Phi}(A) d \mu=\mu(A)
$$

luego:

$$
\mu(A)=\gamma_{S, \lambda}^{\Phi}(A) .
$$

Por lo tanto, si $S$ es finito:

$$
\mathcal{G}\left(\gamma_{\lambda}^{\Phi}\right)=\left\{\gamma_{S, \lambda}^{\Phi}\right\}
$$


Es de interés, dentro de este caso (S finito) la siguiente situación:

E finito, $\lambda$ la medida de conteo.

$\overline{E n}$ este caso para abreviar la notación, dejaremos de lado el símbolo " $\lambda$ " en lo anterior.

Como $E$ y $S$ son finitos; $E^{S}$ también lo es y si $\mathcal{E}=\mathcal{P}(E)$, entonces $\mathcal{F}=\mathcal{P}\left(E^{S}\right)$.

Por otra parte, en este caso, cualquiera sea el potencial $\Phi=\left(\Phi_{\Lambda}\right)_{\Lambda \in \mathcal{S}}$ $(\mathcal{S}=\mathcal{P}(\mathcal{S}) \backslash\{\emptyset\})$ es $\lambda$-admisible. También: $\forall x \in E^{S}$.

$$
\begin{aligned}
H_{S}^{\Phi}(x) & =\sum_{\Lambda \in \mathcal{S}} \Phi_{\Lambda}(x) \\
Z_{S}^{\Phi} & =\sum_{x \in E^{S}} \exp \left(-H_{S}^{\Phi}(x)\right), \\
\varrho_{S}^{\Phi}(x) & =\frac{\exp \left(-H_{S}^{\Phi}(x)\right)}{Z_{S}^{\Phi}}, \\
\gamma_{S}^{\Phi}(\{x\}) & =\varrho_{S}^{\Phi}(x) .
\end{aligned}
$$

Notemos entonces que $\gamma_{S}^{\Phi}(\{x\})>0 \forall x \in E^{S}$.

Sea ahora $\Lambda \in \mathcal{S}, \Lambda \neq S$. Como $\gamma_{\Lambda}^{\Phi}(A \mid \cdot) \in \gamma_{S}^{\Phi}\left(A \mid \mathcal{J}_{\Lambda}\right) \forall A \in E^{S}$, se tiene entonces:

$$
\varrho_{\Lambda}^{\Phi}(y)=\frac{\varrho_{S}^{\Phi}(y)}{\sum_{\xi \in E^{S}} \varrho_{S}^{\Phi}\left(\xi y_{S \backslash \Lambda}\right)}, y \in E^{S} .
$$

En particular, cuando $\Lambda=\{s\}, \varrho_{\Lambda}^{\Phi}(y)$ se llama característica local de y en s asociada a $\gamma_{S}^{\Phi}$.

En general, cuando $E$ y $S$ son finitos y $\mathcal{F}=\mathcal{P}\left(E^{S}\right)$, si $\pi$ es una probabilidad nunca nula sobre $\left(E^{S}, \mathcal{F}\right)$, entonces

$$
\pi_{s}(y):=\frac{\pi(\{y\})}{\sum_{\xi \in E} \pi\left(\left\{\xi y_{S \backslash\{s\}}\right\}\right)},
$$

que por definición es la característica local de $y \in E^{S}$ en s asociada $\boldsymbol{a} \pi$, caracteriza a $\pi$ en el sentido siguiente:

Sean $\pi y \mu$ dos probabilidades nunca nulas sobre $\left(E^{S}, \mathcal{F}\right)$. Si $\pi_{s}(y)=$ $\mu_{s}(y) \forall y \in E^{S}, \forall s \in S$, entonces $\pi=\mu$. (Ver, por ejemplo: Bustos $y$ Ojeda (1994) [3]).

Ejemplo 3.2.6 (Modelo de Ising Anisotrópico). Sean: $E=\{-1,+1\}$; para cada $s=\left(s_{1}, s_{2}\right) \in S$ definimos

$$
\begin{aligned}
& \mathcal{V}_{s, V}^{1}:=\left\{\left(s_{1}, s_{2}-1\right),\left(s_{1}, s_{2}+1\right)\right\} . \\
& \mathcal{V}_{s, H}^{1}:=\left\{\left(s_{1}-1, s_{2}\right),\left(s_{1}+1, s_{2}\right)\right\} .
\end{aligned}
$$


a) Modelo de Ising anisotrópico sin campo externo.

$$
\begin{aligned}
\text { Sean } \beta_{H}, \beta_{V} \in \mathbb{R} ; \Phi^{\left(\beta_{H}, \beta_{V}\right)}=\left(\Phi_{\Lambda}^{\left(\beta_{H}, \beta_{V}\right)}\right)_{\Lambda \in \mathcal{S}} \text { con } \\
\Phi_{\Lambda}^{\left(\beta_{H}, \beta_{V}\right)}(x)= \begin{cases}\beta_{V} x(s) x(t) & \text { si }\{s, t\}=\Lambda \subset \mathcal{V}_{s, V}^{1} \cup\{s\} s \neq t \\
\beta_{H} x(s) x(t) & \text { si }\{s, t\}=\Lambda \subset \mathcal{V}_{s, H}^{1} \cup\{s\} s \neq t \\
0 & \text { c.c. }\end{cases}
\end{aligned}
$$

b) Modelo de Ising anisotrópico con campo externo.

Sea $\alpha, \beta_{H}, \beta_{V} \in \mathbb{R} ; \Phi^{\left(\alpha, \beta_{H}, \beta_{V}\right)}=\left(\Phi_{\Lambda}^{\left(\alpha, \beta_{H}, \beta_{V}\right)}\right)_{\Lambda \in \mathcal{S}}$ con

$$
\Phi_{\Lambda}^{\left(\alpha, \beta_{H}, \beta_{V}\right)}(x)=\left\{\begin{array}{l}
\alpha x(s) \quad \text { si }\{s\}=\Lambda, s \in S \\
\beta_{V} x(s) x(t) \quad \text { si }\{s, t\}=\Lambda \subset \mathcal{V}_{s, V}^{1} \cup\{s\} \quad s \neq t \\
\beta_{H} x(s) x(t) \quad \text { si }\{s, t\}=\Lambda \subset \mathcal{V}_{s, H}^{1} \cup\{s\} \quad s \neq t \\
0 \quad c . c .
\end{array}\right.
$$

Afirmación: $\Phi_{\Lambda}^{\left(\beta_{H}, \beta_{V}\right)} y \Phi_{\Lambda}^{\left(\alpha, \beta_{H}, \beta_{V}\right)}$ son $\mathcal{G}^{\delta}$-potenciales $(\delta \geq 1)$. Luego, por Corolario 3.1.16 si $\lambda$ es la medida de conteo sobre $(E, \mathcal{P}(E))$ tenemos que

$$
\mathcal{G}\left(\gamma_{\lambda}^{\Phi^{\left(\beta_{H}, \beta_{V}\right)}}\right) \neq \emptyset \quad y \quad \mathcal{G}\left(\gamma_{\lambda}^{\Phi^{\left(\alpha, \beta_{H}, \beta_{V}\right)}}\right) \neq \emptyset .
$$

Ejemplo 3.2.7 (Modelo de Potts-Strauss). Sean $E:=\left\{a_{0}, a_{1}, \ldots, a_{k-1}\right\}$ un conjunto finito cualquiera; $\mathcal{E}=\mathcal{P}(E)(k \geq 2)$.

Para cada $t=0,1, \ldots, k-1$ sea $\alpha_{t} \in \mathbb{R}$.

Para cada $0 \leq t<l \leq k-1$ sea $\beta_{(t, l)} \in \mathbb{R}$.

Para cada $\Lambda \in \mathcal{S}$ sea $\Phi_{\Lambda}: E^{S} \rightarrow \mathbb{R}$ dada por

$$
\Phi_{\Lambda}(x)=\left\{\begin{array}{l}
\alpha_{t} \text { si } \Lambda=\{i\} \text { y } x(i)=a_{t} \\
\beta_{(t, l)} \text { si } \Lambda=\{i, j\} \text { con } j \in \mathcal{V}_{i}^{1} \text { y }\{x(i), x(j)\}=\left\{a_{t}, a_{l}\right\} \\
0 \quad \text { c.c. }
\end{array}\right.
$$

Si $\lambda$ es la medida de conteo sobre $(E, \mathcal{P}(E))$, por Corolario 3.1.16 tenemos que

$$
\mathcal{G}\left(\gamma_{\lambda}^{\Phi}\right) \neq \emptyset \quad \operatorname{con} \gamma_{\lambda}^{\Phi}:=\left(\gamma_{\Lambda, \lambda}^{\Phi}\right)_{\Lambda \in \mathcal{S}} .
$$

Ejemplo 3.2.8 (Un Potencial Paramétrico General). Sea E y $S$ como al inicio de este Capítulo. Sea $\mathcal{C} \subset \mathcal{S}$ (no vacía).

Para cada $C \in \mathcal{S}$ sean: $\theta_{C} \in \mathbb{R}^{p_{c}} ; \phi_{C}: E^{S} \rightarrow \mathbb{R}^{p_{c}} \mathcal{F}_{C}$-medible, con $p_{c} \geq 1$ entero.

Para cada $\Lambda \in \mathcal{S}$ sea:

$$
\begin{aligned}
\Phi_{\Lambda} & \equiv 0 \quad \text { si } \Lambda \notin \mathcal{C} \\
\Phi_{C}(x) & =\theta_{C}^{t} \phi_{C}(x) \quad \text { con } x \in E^{S}, C \in \mathcal{C} .
\end{aligned}
$$

$A$ los fines de inferencia se supone que $\phi_{C}$ es conocida, $\forall C \in \mathcal{C}$. 


\subsection{Potenciales Normalizados e Identificables}

Definición 3.3.1. Sean: $\Phi=\left(\Phi_{\Lambda}\right)_{\Lambda \in \mathcal{S}}$ un potencial sobre $\left(E^{S}, \mathcal{F}\right) ; a \in E$. Se dice que $\Phi$ es a-normalizado si es cierta la siguiente propiedad: $\left(x \in E^{S}, \exists s \in \Lambda\right.$ tal que $\left.x(s)=a\right) \Rightarrow \Phi_{\Lambda}(x)=0$.

Definición 3.3.2. Se dice que $f: E^{S} \rightarrow \mathbb{R}$ es local si $\exists \Lambda \in \mathcal{S}$ tal que $f$ es $\mathcal{F}_{\Lambda}$-medible.

Nota 3.3.3. Si $S$ es finito, entonces toda $f: E^{S} \rightarrow \mathbb{R} \mathcal{F}$-medible es local.

Notación 3.3.4. Para cada $\Lambda \in \mathcal{S}$ sea

$$
\mathcal{L}_{\Lambda}:=\left\{f: E^{S} \rightarrow \mathbb{R} / f \text { es acotada y } \mathcal{F}_{\Lambda} \text {-medible }\right\} .
$$

Sea

$$
\mathcal{L}:=\bigcup_{\Lambda \in \mathcal{S}} \mathcal{L}_{\Lambda}
$$

Definición 3.3.5. Se dice que $f: E^{S} \rightarrow \mathbb{R} \mathcal{F}$-medible es quasilocal si $\exists\left(f_{n}\right)_{n \geq 1}$ sucesión de funciones locales tal que:

1. $f-f_{n} \in \mathcal{L}^{\infty}\left(E^{S}, \mathcal{F}, \mathbb{R}\right), \forall n \geq 1$.

2. $\left\|f-f_{n}\right\|_{\mathcal{L}^{\infty}} \underset{n \rightarrow \infty}{\longrightarrow} 0$.

Proposición 3.3.6. Sea $f \in \mathcal{L}^{\infty}\left(E^{S}, \mathcal{F}, \mathbb{R}\right)$. Entonces $f$ es quasilocal si $y$ sólo si $f \in \overline{\mathcal{L}}$ (clausura de $\mathcal{L}$ en $\mathcal{L}^{\infty}\left(E^{S}, \mathcal{F}, \mathbb{R}\right)$ ).

Demostración. Ejercicio.

Notación 3.3.7. Sea $f: E^{S} \rightarrow \mathbb{R}$ (función cualquiera); $\Lambda \in \mathcal{S}$ ponemos:

$$
O_{\Lambda}(f):=\sup \left(\left\{\left|f(x)-f\left(x^{\prime}\right)\right| / x_{\Lambda}=x_{\Lambda}^{\prime}\right\}\right) .
$$

Notemos que si $\Lambda_{1} \subset \Lambda_{2}$ y ambos están en $\mathcal{S}$, entonces: $O_{\Lambda_{2}}(f) \leq$ $O_{\Lambda_{1}}(f)$.

Proposición 3.3.8. Sea $f: E^{S} \rightarrow \mathbb{R} \mathcal{F}$-medible. Entonces $f$ es quasilocal si y sólo si

$$
O(f):=\inf _{\Lambda \in \mathcal{S}} O_{\Lambda}(f)=0 .
$$

Demostración. $\Rightarrow\rfloor$ Sea $\varepsilon>0$ cualquiera. Como $f$ es quasilocal, $\exists \Lambda \in \mathcal{S}$, $\phi_{\Lambda}: E^{\Lambda} \rightarrow \mathbb{R} \mathcal{E}^{\Lambda}$-medible tal que

$$
\left\|f-\phi_{\Lambda} \circ \sigma_{\Lambda}\right\|_{\mathcal{L}^{\infty}}<\varepsilon / 2 .
$$

Sean ahora $x$ y $x^{\prime}$ en $E^{S}$ tales que $x_{\Lambda}=x_{\Lambda}^{\prime} \Rightarrow\left(\phi_{\Lambda} \circ \sigma_{\Lambda}\right)(x)=$ $\left(\phi_{\Lambda} \circ \sigma_{\Lambda}\right)\left(x^{\prime}\right)$. 
Luego

$\left|f(x)-f\left(x^{\prime}\right)\right| \leq\left|f(x)-\left(\phi_{\Lambda} \circ \sigma_{\Lambda}\right)(x)\right|+\left|f\left(x^{\prime}\right)-\left(\phi_{\Lambda} \circ \sigma_{\Lambda}\right)\left(x^{\prime}\right)\right|<\frac{\varepsilon}{2}+\frac{\varepsilon}{2}=\varepsilon$.

Luego $O_{\Lambda}(f) \leq \varepsilon$ y por lo tanto $O(f) \leq \varepsilon$.

Por la arbitrariedad de $\varepsilon$ se sigue que $O(f)=0$.

$\Leftarrow\rfloor$ Sea $\epsilon>0$ cualquiera.

Sea $\Lambda_{1} \in \mathcal{S}$ tal que $O_{\Lambda_{1}}(f)<\epsilon$.

Sea $\Lambda \in \mathcal{S}$ tal que $\Lambda_{1} \subset \Lambda \Rightarrow O_{\Lambda}(f)<\epsilon$

Sea $x \in E^{S}$ y $\phi_{\Lambda}: E^{\Lambda} \rightarrow \mathbb{R}$ definida por

$$
\phi_{\Lambda}(\xi)=f\left(\xi x_{S \backslash \Lambda}\right) \quad \forall \xi \in E^{\Lambda} .
$$

Luego $\phi_{\Lambda} \circ \sigma_{\Lambda}$ es local y $\forall z \in E^{S}$ tenemos

$$
\left|f(z)-\left(\phi_{\Lambda} \circ \sigma_{\Lambda}\right)(z)\right|=\left|f\left(z_{\Lambda} z_{S \backslash \Lambda}\right)-f\left(z_{\Lambda} x_{S \backslash \Lambda}\right)\right| \leq O_{\Lambda}(f)<\epsilon .
$$

Luego

$$
\left\|f-\phi_{\Lambda} \circ \sigma_{\Lambda}\right\|_{\mathcal{L}^{\infty}}<\epsilon .
$$

Por lo tanto, $f$ es quasilocal.

Nota 3.3.9. Asumimos sin demostración los dos siguientes resultados. Su demostración puede verse en el libro: Georgii (1998)[6], pag.32.

Nota 3.3.10. a) Si E es separable (no necesariamente completo), entonces:

$$
f: E^{S} \rightarrow \mathbb{R} \text { uniformemente continua } \Rightarrow f \text { es quasilocal. }
$$

b) Si E es finito, entonces

$$
f: E^{S} \rightarrow \mathbb{R} \text { uniformemente continua } \Leftrightarrow f \text { es quasilocal. }
$$

De ahora en adelante en esta Sección sean: $\lambda$ una medida $\sigma$-finita sobre $(E, \mathcal{E}) ; \Phi=\left(\Phi_{\Lambda}\right)_{\Lambda \in \mathcal{S}}$ un potencial sobre $\left(E^{S}, \mathcal{F} F\right) \lambda$-admisible; $\left(\varrho_{\Lambda, \lambda}^{\Phi}\right)_{\Lambda \in \mathcal{S}}$, $\left(Z_{\Lambda, \lambda}^{\Phi}\right)_{\Lambda \in \mathcal{S}}$ y $\left(\gamma_{\Lambda, \lambda}^{\Phi}\right)_{\Lambda \in \mathcal{S}}$ como en la Proposición 3.1.3.

Pondremos:

$$
\gamma_{\lambda}^{\Phi}:=\left(\gamma_{\Lambda, \lambda}^{\Phi}\right)_{\Lambda \in \mathcal{S}}
$$

Sea $f: E^{S} \rightarrow \mathbb{R} \mathcal{F}$-medible. Sean $\Lambda \in \mathcal{S}, x \in E^{S}$, siempre que tenga sentido pondremos:

$$
\gamma_{\Lambda, \lambda}^{\Phi}(f)(x):=\int_{E^{\Lambda}} f\left(\xi x_{S \backslash \Lambda}\right) \varrho_{\Lambda, \lambda}^{\Phi}\left(\xi x_{S \backslash \Lambda}\right) \lambda^{\Lambda}(d \xi) .
$$


Definición 3.3.11. Se dice que $\gamma_{\lambda}^{\Phi}$ es quasilocal si:

$$
\Lambda \in \mathcal{S}, f \in \mathcal{L} \Rightarrow \gamma_{\Lambda, \lambda}^{\Phi}(f) \in \overline{\mathcal{L}}
$$

Nota 3.3.12. En la misma pag. 32 del ya citado libro de Georgii se puede probar la siguiente afirmación:

Se cumplen las siguientes propiedades:

a) $\varrho_{\Lambda, \lambda}^{\Phi}$ local $\forall \Lambda \in \mathcal{S} \Rightarrow \gamma_{\lambda}^{\Phi}$ es quasilocal.

b) $\varrho_{\Lambda, \lambda}^{\Phi}$ quasilocal $\forall \Lambda \in \mathcal{S}$ y $\lambda(E)<\infty \Rightarrow \gamma_{\lambda}^{\Phi}$ es quasilocal.

c) $H_{\Lambda}^{\Phi}$ local $\forall \Lambda \in \mathcal{S} \Rightarrow \gamma_{\lambda}^{\Phi}$ es quasilocal.

d) Supongamos: $E$ finito, $\lambda$ la medida de conteo, $\gamma$ una especificación sobre $\left(E^{S}, \mathcal{P}(E)^{S}\right)$. Para cada $\Lambda \in \mathcal{S}$ sea $\rho_{\Lambda}: E^{S} \rightarrow \mathbb{R}$ dada por

$$
\rho_{\Lambda}(x)=\gamma_{\Lambda}\left(1_{\sigma_{\Lambda}^{-1}\left(\left\{x_{\Lambda}\right\}\right)}\right)(x)
$$

Si $\gamma$ es quasilocal, entonces $\rho_{\Lambda} \in \overline{\mathcal{L}} \forall \Lambda \in \mathcal{S}$.

Definición 3.3.13. $\Phi=\left(\Phi_{\Lambda}\right)_{\Lambda \in \mathcal{S}}$ un potencial . Se dice que $\Phi$ es de rango finito si:

$$
\begin{gathered}
\text { para cada } s \in S, \exists \Lambda_{s} \in \mathcal{S} \text { tal que: } \\
s \in \Delta \in S \text { y } \Delta \cap \Lambda_{s}^{c} \neq \emptyset \Rightarrow \Phi_{\Delta} \equiv 0 .
\end{gathered}
$$

Proposición 3.3.14. Sean: $\lambda$ una medida $\sigma$-finita sobre $(E, \mathcal{E}) ; \Phi=$ $\left(\Phi_{\Lambda}\right)_{\Lambda \in \mathcal{S}}$ un potencial sobre $\left(E^{S}, \mathcal{F}\right) \lambda$-admisible de rango finito. Entonces $\varrho_{\Lambda, \lambda}^{\Phi}$ es local $\forall \Lambda \in \mathcal{S}$.

Demostración. Sea $\Lambda \in \mathcal{S}$ cualquiera y sea $\Delta \in \mathcal{S} \cap \Lambda$ tal que $\Phi_{\Delta} \not \equiv 0$. Luego:

$$
\Lambda \subset \bigcup_{s \in \Lambda} \Lambda_{s}=: \Lambda^{*} \in \mathcal{S} .
$$

Por consiguiente, $H_{\Lambda}^{\Phi}$ es $\mathcal{F}_{\Lambda^{*}-\text { medible. De aquí se deduce fácilmente que }}$ $\varrho_{\Lambda, \lambda}^{\Phi}$ es $\mathcal{F}_{\Lambda^{*}-\text { medible. }}$

Proposición 3.3.15. Sean: $\mathcal{V}$ un sistema de vecindades de $S, \mathcal{G}=(S, \mathcal{V})$, $\Phi=\left(\Phi_{\Lambda}\right)_{\Lambda \in \mathcal{S}}$ un $\mathcal{G}$-potencial. Entonces $\Phi$ es de rango finito.

Demostración. Ejercicio.

Corolario 3.3.16. Sean: $\lambda$ una medida $\sigma$-finita, $\mathcal{V}$ un sistema de vecindades de $S, \mathcal{G}=(S, \mathcal{V}), \Phi=\left(\Phi_{\Lambda}\right)_{\Lambda \in \mathcal{S}}$ un $\mathcal{G}$-potencial $\lambda$-admisible. Entonces $\varrho_{\Lambda, \lambda}^{\Phi}$ es local $\forall \Lambda \in \mathcal{S}$. Luego $\gamma_{\lambda}^{\Phi}$ es quasilocal. 
Definición 3.3.17. Sea $\Phi=\left(\Phi_{\Lambda}\right)_{\Lambda \in \mathcal{S}}$ un potencial sobre $\left(E^{S}, \mathcal{F}\right)$. Se dice que $\Phi$ es uniformemente convergente (en simbolos u.c.) si para cada $\Lambda_{0} \in \mathcal{S}$ se cumple:

$$
\begin{gathered}
\text { Dado } \varepsilon>0, \exists \Delta_{0} \in \mathcal{S} \text { tal que } \\
\Delta_{0} \subset \Delta \in \mathcal{S} \Rightarrow\left|H_{\Lambda_{0}}^{\Phi}(x)-\sum_{\Lambda \in \mathcal{S} \cap \Lambda_{0}, \Lambda \subset \Delta} \Phi_{\Lambda}(x)\right|<\varepsilon, \forall x \in E^{S} .
\end{gathered}
$$

Proposición 3.3.18. Sea $\Phi=\left(\Phi_{\Lambda}\right)_{\Lambda \in \mathcal{S}}$ un potencial sobre $\left(E^{S}, \mathcal{F}\right)$. Si $\Phi$ es de rango finito, entonces $\Phi$ es u.c.

Demostración. Ejercicio.

Nota 3.3.19. Por esta última Proposición y por la Proposicion 3.3.15 se deduce que:

Sean: $\mathcal{V}$ un sistema de vecindades de $S, \mathcal{G}=(S, \mathcal{V}), \Phi=\left(\Phi_{\Lambda}\right)_{\Lambda \in \mathcal{S}}$ un $\mathcal{G}$-potencial. Entonces $\Phi$ es u.c.

Definición 3.3.20. Sean $\Phi=\left(\Phi_{\Lambda}\right)_{\Lambda \in \mathcal{S}} y \Psi=\left(\Psi_{\Lambda}\right)_{\Lambda \in \mathcal{S}}$ dos potenciales sobre $\left(E^{S}, \mathcal{F}\right)$. Se dice que $\Psi$ y $\Phi$ son equivalentes (en símbolos $\Psi \sim \Phi$ ) si:

$$
\Lambda \in \mathcal{S} \Rightarrow H_{\Lambda}^{\Phi}-H_{\Lambda}^{\Psi} \text { es } \mathcal{F}_{S \backslash \Lambda} \text {-medible. }
$$

Proposición 3.3.21. Sean $a \in E ; \Phi=\left(\Phi_{\Lambda}\right)_{\Lambda \in \mathcal{S}} y \Psi=\left(\Psi_{\Lambda}\right)_{\Lambda \in \mathcal{S}}$ dos potenciales sobre $\left(E^{S}, \mathcal{F}\right)$ a-normalizados. Si $\Psi \sim \Phi$, entonces $\Psi_{\Lambda}=\Phi_{\Lambda}$ $\forall \Lambda \in \mathcal{S}$.

Demostración. Procedemos por inducción sobre \# $(\Lambda)$.

Caso 1: $s \in \mathcal{S}, \Lambda:=\{s\}$.

Sea $x \in E^{s}$.

Sea

$$
y=x_{s} a_{S \backslash\{s\}} .
$$

Esto es:

$$
\sigma_{t}(y)= \begin{cases}a & \forall t \neq s \\ x_{s} & t=s .\end{cases}
$$

Como $\Phi_{\Lambda}$ y $\Psi_{\Lambda}$ son $\mathcal{F}_{\Lambda}$-medibles, $\exists \phi: E^{\Lambda} \rightarrow \mathbb{R}$ y $\psi: E^{\Lambda} \rightarrow \mathbb{R} \mathcal{E}^{\Lambda_{-}}$ medibles tales que: $\Phi_{\Lambda}=\phi \circ \sigma_{\Lambda} \quad$ y $\quad \Psi_{\Lambda}=\psi \circ \sigma_{\Lambda}$.

Luego

$$
\Phi_{\Lambda}(x)=\Phi_{\Lambda}(y) \quad y \quad \Psi_{\Lambda}(x)=\Psi_{\Lambda}(y) .
$$

Sea ahora $\Delta \in \mathcal{S} \cap \Lambda$ con $\#(\Delta) \geq 2$. Como $\Phi=\left(\Phi_{\Lambda}\right)_{\Lambda \in \mathcal{S}}$ y $\Psi=$ $\left(\Psi_{\Lambda}\right)_{\Lambda \in \mathcal{S}}$ son $a$-normalizados se tiene que

$$
\Phi_{\Delta}(y)=\Psi_{\Delta}(y)=0
$$


Luego:

$$
H_{\Lambda}^{\Phi}(y)=\Phi_{\Lambda}(y) \quad \text { y } \quad H_{\Lambda}^{\Psi}(y)=\Psi_{\Lambda}(y) .
$$

Como $H_{\Lambda}^{\Phi}-H_{\Lambda}^{\Psi}$ es $\mathcal{F}_{S \backslash \Lambda}$-medible, tenemos que:

$H_{\Lambda}^{\Phi}\left(\xi y_{S \backslash \Lambda}\right)-H_{\Lambda}^{\Psi}\left(\xi y_{S \backslash \Lambda}\right)=H_{\Lambda}^{\Phi}\left(\eta y_{S \backslash \Lambda}\right)-H_{\Lambda}^{\Psi}\left(\eta y_{S \backslash \Lambda}\right), \quad \forall \xi, \eta \in E$.

Como $\Phi=\left(\Phi_{\Lambda}\right)_{\Lambda \in \mathcal{S}}$ y $\Psi=\left(\Psi_{\Lambda}\right)_{\Lambda \in \mathcal{S}}$ son $a$-normalizados se tiene que:

$$
\begin{aligned}
& H_{\Lambda}^{\Phi}\left(a y_{S \backslash \Lambda}\right)=H_{\Lambda}^{\Phi}(\tilde{a})=0 \\
& H_{\Lambda}^{\Psi}\left(a y_{S \backslash \Lambda}\right)=H_{\Lambda}^{\Psi}(\tilde{a})=0
\end{aligned}
$$

donde $\tilde{a}$ es el elemento de $E^{S}$ dado por

$$
\sigma_{t}(\tilde{a})=a \quad \forall t \in S .
$$

Luego por (4) con $\eta=a$, tenemos que

$$
H_{\Lambda}^{\Phi}(y)-H_{\Lambda}^{\Psi}(y)=0 .
$$

Luego, por (3):

$$
\Psi_{\Lambda}(y)=\Phi_{\Lambda}(y)
$$

y por (1):

$$
\Psi_{\Lambda}(x)=\Phi_{\Lambda}(x) .
$$

La Proposición está probada para el Caso 1.

Caso General: $\#(\Lambda)=n \geq 2$ y por hipótesis inductiva supongamos que

$$
\Phi_{\Delta}=\Psi_{\Delta} \quad \forall \Delta \in \mathcal{S} \text { con } \#(\Delta) \leq n-1
$$

Sea $x \in E^{s}$.

Sea

$$
y=x_{\Lambda} a_{S \backslash \Lambda},
$$

razonando como en el Caso 1 , tenemos:

$$
\Phi_{\Lambda}(x)=\Phi_{\Lambda}(y) \quad \text { y } \quad \Psi_{\Lambda}(x)=\Psi_{\Lambda}(y) .
$$

También:

$$
\begin{aligned}
& H_{\Lambda}^{\Phi}(y)=\sum_{\substack{\Delta \in \mathcal{S} \\
\Delta \subset \Lambda}} \Phi_{\Delta}(y) \\
& H_{\Lambda}^{\Psi}(y)=\sum_{\substack{\Delta \in \mathcal{S} \\
\Delta \subset \Lambda}} \Psi_{\Delta}(y) .
\end{aligned}
$$

Por la hipótesis inductiva (5), tenemos:

$$
H_{\Lambda}^{\Phi}(y)-H_{\Lambda}^{\Psi}(y)=\Phi_{\Lambda}(y)-\Psi_{\Lambda}(y) .
$$


Como $H_{\Lambda}^{\Phi}-H_{\Lambda}^{\Psi}$ es $\mathcal{F}_{S \backslash \Lambda}$-medible:

$$
\left(H_{\Lambda}^{\Phi}-H_{\Lambda}^{\Psi}\right)(y)=H_{\Lambda}^{\Phi}(\tilde{a})-H_{\Lambda}^{\Psi}(\tilde{a})=0 .
$$

Luego por (7):

$$
\Psi_{\Lambda}(y)=\Phi_{\Lambda}(y)
$$

Y por (6) queda probado entonces

$$
\Psi_{\Lambda}(x)=\Phi_{\Lambda}(x) .
$$

Nota 3.3.22. Sean: $A \in \mathcal{S} \cup\{\emptyset\}, a \in E$. Pondremos $\mathcal{F}(a, A)$ para denotar el conjunto de todas las funciones $f: E^{S} \rightarrow \mathbb{R}$ tales que $f_{C}^{a}$ es $\mathcal{F}_{C}$-medible, $\forall C \subset A$ siendo $f_{C}^{a}: E^{S} \rightarrow \mathbb{R}$ definida por

$$
f_{C}^{a}(x)=f\left(x_{C} a_{S \backslash C}\right), \forall x \in E^{S} .
$$

En esta notación si $C=\emptyset$ ponemos:

$$
f_{\emptyset}^{a}(x)=f(\tilde{a}) \quad \forall x \in E^{S},
$$

con ã el elemento de $E^{S}$ dado por:

$$
\tilde{a}(s)=a \quad \forall s \in S .
$$

Por el Teorema de Fubini se tiene que si $f: E^{S} \rightarrow \mathbb{R}$ es $\mathcal{F}$-medible, entonces $f \in \mathcal{F}(a, A)$.

Para cada $f \in \mathcal{F}(a, A)$ definimos.

$$
p_{(a, A)}(f)(x):=\sum_{C \subset A}(-1)^{\#(A \backslash C)} f_{C}^{a}(x) \quad \forall x \in E^{S} .
$$

Proposición 3.3.23. Sean: $a \in E ; A \in \mathcal{S} \cup\{\emptyset\}$. Entonces

a) $f \in \mathcal{F}(a, A) \Rightarrow p_{(a, A)}(f)$ es $\mathcal{F}_{A}$-medible $\left(\mathcal{F}_{\emptyset}:=\left\{\emptyset, E^{S}\right\}\right)$.

b) $f \in \mathcal{F}(a, A) \Rightarrow f_{A}^{a}(x)=\sum_{C \subset A} p_{(a, C)}(f)(x) \quad \forall x \in E^{S}$.

c) $\emptyset \neq B \subset A \Rightarrow p_{(a, A)}(f)_{S \backslash B}^{a}(x)=0, \quad \forall x \in E^{S} \forall f \mathcal{F}-$ medible.

\section{Demostración.}

a) es fácil.

b) se demuestra aplicando la proposición de más abajo (Fórmula de Möbius) con

$$
\Phi(A):=p_{(a, A)}(f)(x)
$$

y

$$
\Psi(A):=f_{A}^{a}(x), x \in E^{S} \text { fijo cualquiera. }
$$


Proposición 3.3.24 (Fórmula de Möbius). Sean $\Phi: \mathcal{S} \cup\{\emptyset\} \rightarrow \mathbb{R}$ $y \Psi: \mathcal{S} \cup\{\emptyset\} \rightarrow \mathbb{R}$. Son equivalentes:

$$
\Phi(A)=\sum_{B \subset A}(-1)^{\#(A \backslash B)} \Psi(B), A \in \mathcal{S} \cup\{\emptyset\}
$$

$y$

$$
\Psi(A)=\sum_{B \subset A} \Phi(B), A \in \mathcal{S} \cup\{\emptyset\} .
$$

La demostración de estas fórmulas queda como ejercicio.

c) Notemos que es suficiente probarla para el caso $\#(B)=1$.

Sea entonces $B=\{s\} \subset A$ con $s \in S$ cualquiera. Sean $x \in E^{S}$ cualquiera y pongamos $y=a_{B} x_{S \backslash B}$.

Entonces

$$
\begin{gathered}
p_{(a, A)}(f)_{S \backslash B}^{a}(x)=p_{(a, A)}(f)(y)= \\
\sum_{s \notin C \subset A}\left[(-1)^{\#(A \backslash C)} f_{C}^{a}(y)+(-1)^{\#(A \backslash(C \cup\{s\}))} f_{C \cup\{s\}}^{a}(y)\right]=(1) .
\end{gathered}
$$

Ahora es fácil ver que

$$
s \notin C \Rightarrow f_{C}^{a}(y)=f_{C}^{a}(x) \text { y } f_{C \cup\{s\}}^{a}(y)=f_{C}^{a}(x) .
$$

Luego

$$
(1)=\sum_{s \notin C \subset A}\left[(-1)^{\#(A \backslash C)} f_{C}^{a}(x)+(-1)^{\#(A \backslash C)} f_{C}^{a}(x)\right]=0 .
$$

Hasta el final de esta Sección sean:

- $\lambda$ una medida $\sigma$-finita sobre $(E, \mathcal{E})$.

- $\Phi=\left(\Phi_{\Lambda}\right)_{\Lambda \in \mathcal{S}}$ un potencial sobre $\left(E^{S}, \mathcal{F}\right) \lambda$-admisible.

- Para cada $\Lambda \in \mathcal{S}$ y cada $x \in E^{S}$, existe en $\mathbb{R}$

$$
H_{\Lambda}^{\Phi}(x):=\sum_{\Delta \in \mathcal{S} \cap \Lambda} \Phi_{\Delta}(x) .
$$

- $Z_{\Lambda, \lambda}^{\Phi}(x)=\int_{E^{\Lambda}} \exp \left(-H_{\Lambda}^{\Phi}\left(\xi x_{S \backslash \Lambda}\right)\right) \lambda^{\Lambda}(d \xi)$.

$\varrho_{\Lambda, \lambda}^{\Phi}(x)=\frac{\exp \left(-H_{\Lambda}^{\Phi}(x)\right)}{Z_{\Lambda, \lambda}^{\Phi}(x)}$.

$\gamma_{\Lambda, \lambda}^{\Phi}(A \mid x):=\int_{E^{\Lambda}} 1_{A}\left(\xi x_{S \backslash \Lambda}\right) \varrho_{\Lambda, \lambda}^{\Phi}\left(\xi x_{S \backslash \Lambda}\right) \lambda^{\Lambda}(d \xi) A \in \mathcal{F}$.

$\gamma_{\lambda}^{\Phi}=\left(\gamma_{\Lambda, \lambda}^{\Phi}\right)_{\Lambda \in \mathcal{S}}$. 
Proposición 3.3.25. Sean: $a \in E, \Lambda \in \mathcal{S}, \Delta \in \mathcal{S}, \Lambda \subset \Delta$.

a) $\ln \left(\varrho_{\Lambda}^{\Phi}\right)\left(a_{\Lambda} x_{S \backslash \Lambda}\right)-\ln \left(\varrho_{\Lambda}^{\Phi}\right)(x)=$

$$
\begin{aligned}
& H_{\Lambda}^{\Phi}(x)-H_{\Lambda}^{\Phi}\left(a_{\Lambda} x_{S \backslash \Lambda}\right)= \\
& H_{\Delta}^{\Phi}(x)-H_{\Delta}^{\Phi}\left(a_{\Lambda} x_{S \backslash \Lambda}\right)= \\
& \ln \left(\varrho_{\Delta}^{\Phi}\right)\left(a_{\Lambda} x_{S \backslash \Lambda}\right)-\ln \left(\varrho_{\Delta}^{\Phi}\right)(x) x \in E^{S} . \\
& \text { b) } p_{(a, \Lambda)}\left(\ln \left(\varrho_{\Lambda}^{\Phi}\right)\right)=p_{(a, \Lambda)}\left(\ln \left(\varrho_{\Delta}^{\Phi}\right)\right)
\end{aligned}
$$

\section{Demostración.}

$\underline{\text { Parte a) }} \ln \left(\varrho_{\Lambda}^{\Phi}\right)\left(a_{\Lambda} x_{S \backslash \Lambda}\right)-\ln \left(\varrho_{\Lambda}^{\Phi}\right)(x)=\ln \left(\frac{\varrho_{\Lambda}^{\Phi}\left(a_{\Lambda} x_{S \backslash \Lambda}\right)}{\varrho_{\Lambda}^{\Phi}(x)}\right)$

$$
=\ln \left(\frac{\frac{\exp \left(-H_{\Lambda}^{\Phi}\left(a_{\Lambda} x_{S} \backslash \Lambda\right.\right.}{Z_{\Lambda, \lambda}\left(a_{\Lambda} x_{S \backslash \Lambda}\right)}}{\frac{\exp \left(-H_{\Lambda}^{\phi}(x)\right)}{Z_{\Lambda, \lambda}^{\Phi}(x)}}\right)=(1)
$$

Como $Z_{\Lambda, \lambda}^{\Phi}$ es $\mathcal{F}_{S \backslash \Lambda}$-medible, tenemos que

$$
Z_{\Lambda, \lambda}^{\Phi}\left(a_{\Lambda} x_{S \backslash \Lambda}\right)=Z_{\Lambda, \lambda}^{\Phi}(x) .
$$

Luego

$$
(1)=\ln \left(\frac{\exp \left(-H_{\Lambda}^{\Phi}\left(a_{\Lambda} x_{S \backslash \Lambda}\right)\right)}{\exp \left(-H_{\Lambda}^{\Phi}(x)\right)}\right)=H_{\Lambda}^{\Phi}(x)-H_{\Lambda}^{\Phi}\left(a_{\Lambda} x_{S \backslash \Lambda}\right) .
$$

Como $\Lambda \subset \Delta, S \backslash \Delta \subset S \backslash \Lambda$. Luego $Z_{\Delta, \lambda}^{\Phi}$ es $\mathcal{F}_{S \backslash \Lambda}$-medible, pues es $\mathcal{F}_{S \backslash \Delta}$-medible.

Por lo tanto, con un razonamiento similar al de recién, se prueba que:

$\ln \left(\varrho_{\Delta}^{\Phi}\right)\left(a_{\Lambda} x_{S \backslash \Lambda}\right)-\ln \left(\varrho_{\Delta}^{\Phi}\right)(x)=H_{\Delta}^{\Phi}(x)-H_{\Delta}^{\Phi}\left(a_{\Lambda} x_{S \backslash \Lambda}\right)$.

Ahora

$$
H_{\Delta}^{\Phi}(x)-H_{\Lambda}^{\Phi}(x)=\sum_{A \in \mathcal{S} \cap \Delta} \Phi_{A}(x)-\sum_{A \in \mathcal{S} \cap \Lambda} \Phi_{A}(x)=(2) .
$$

Ahora $\mathcal{S} \cap \Lambda \subset \mathcal{S} \cap \Delta$. Luego:

$$
(2)=\sum_{\substack{A \in \mathcal{S} \cap \Delta \\ A \subset S \backslash \Lambda}} \Phi_{A}(x)=(3) .
$$

Como $\Phi_{A}$ es $\mathcal{F}_{A}$-medible, se sigue que (3) es $\mathcal{F}_{S \backslash \Lambda}$-medible. Luego:

$$
(3)=\sum_{\substack{A \in \mathcal{S} \cap \Delta \\ A \subset S \backslash \Lambda}} \Phi_{A}(x)=H_{\Delta}^{\Phi}\left(a_{\Lambda} x_{S \backslash \Lambda}\right)-H_{\Lambda}^{\Phi}\left(a_{\Lambda} x_{S \backslash \Lambda}\right) .
$$


Por lo tanto:

$$
H_{\Delta}^{\Phi}(x)-H_{\Delta}^{\Phi}\left(a_{\Lambda} x_{S \backslash \Lambda}\right)=H_{\Lambda}^{\Phi}(x)-H_{\Lambda}^{\Phi}\left(a_{\Lambda} x_{S \backslash \Lambda}\right) .
$$

Con lo cual a) está probada.

$\underline{\text { Parte b) }} \quad p_{(a, \Lambda)}\left(\ln \left(\varrho_{\Lambda}^{\Phi}\right)\right)(x)-p_{(a, \Lambda)}\left(\ln \left(\varrho_{\Delta}^{\Phi}\right)\right)(x)=$

$$
\sum_{C \subset \Lambda}(-1)^{\#(\Lambda \backslash C)}\left(\ln \left(\varrho_{\Lambda}^{\Phi}\right)\left(x_{C} a_{S \backslash C}\right)-\ln \left(\varrho_{\Delta}^{\Phi}\right)\left(x_{C} a_{S \backslash C}\right)\right)=(4) .
$$

Ahora, sea $C \subset \Lambda$. Sea $y=x_{C} a_{S \backslash C}$. Por lo visto en a):

$$
\ln \left(\varrho_{\Lambda}^{\Phi}\right)-\ln \left(\varrho_{\Lambda}^{\Phi}\right)=-\left(\ln \left(\varrho_{\Lambda}^{\Phi}\right)\left(a_{\Lambda} y_{S \backslash \Lambda}\right)-\ln \left(\varrho_{\Delta}^{\Phi}\right)\left(a_{\Lambda} y_{S \backslash \Lambda}\right)\right) .
$$

Pero $a_{\Lambda} y_{S \backslash \Lambda}=\tilde{a}$ pues $C \subset \Lambda$. Entonces

$$
(4)=-\left(\ln \left(\varrho_{\Lambda}^{\Phi}\right)(a)-\ln \left(\varrho_{\Delta}^{\Phi}\right)(a)\right) \sum_{C \subset \Lambda}(-1)^{\#(\Lambda \backslash C)}=0
$$

pues $\sum_{C \subset \Lambda}(-1)^{\#(\Lambda \backslash C)}=0$.

Notación 3.3.26. Sean: $\Lambda \in \mathcal{S}, x \in E^{S}, f: E^{S} \longmapsto \mathbb{R}$. Pondremos

$$
O_{\Lambda}(f)(x):=\sup _{\xi \in E^{S \backslash \Lambda}}\left|f\left(x_{\Lambda} \xi\right)-f(x)\right| .
$$

Notemos que:

$$
\Lambda_{1} \subset \Lambda_{2} \Rightarrow O_{\Lambda_{2}}(f)(x) \leq O_{\Lambda_{1}}(f)(x) .
$$

Definición 3.3.27. Sea $x \in E^{S}, f: E^{S} \longmapsto \mathbb{R}$. Llamaremos oscilación de $f$ en $x$ al infinito a:

$$
O_{\infty}(f)(x):=\inf _{\Lambda \in \mathcal{S}} O_{\Lambda}(f)(x) .
$$

Ejercicio 3.3.28. Para cada $n=1,2, \ldots$ sea $\Lambda_{n} \in \mathcal{S}$ y tal que:

- $\Lambda_{1} \subset \Lambda_{2} \subset \ldots y$

- $\Lambda \in \mathcal{S} \Rightarrow \exists n$ tal que $\Lambda \subset \Lambda_{n}$.

Entonces

$$
O_{\infty}(f)(x)=\lim _{n \rightarrow \infty} O_{\Lambda_{n}}(f)(x)
$$


Teorema 3.3.29. Supongamos que

$$
O_{\infty}\left(\varrho_{\Lambda}^{\Phi}\right)(x)=0, \quad x \in E^{S}, \Lambda \in \mathcal{S} .
$$

Sea $a \in E$, para cada $\Lambda \in \mathcal{S}$ sea $\Phi_{\Lambda}^{a}: E^{S} \longmapsto \mathbb{R}$ dada por

$$
\begin{aligned}
\Phi_{\Lambda}^{a}(x) & =-p_{(a, \Lambda)}\left(\ln \left(\varrho_{\Lambda}^{\Phi}\right)\right)(x) \\
& =-\sum_{C \subset \Lambda}(-1)^{\#(\Lambda \backslash C)}\left(\ln \left(\varrho_{\Lambda}^{\Phi}\right)\right)_{C}^{a}(x) .
\end{aligned}
$$

Para cada $\Lambda \in \mathcal{S}, \Delta \in \mathcal{S}$ con $\Lambda \subset \Delta$ sea

$$
H_{\Lambda, \Delta}^{\Phi^{a}}(x):=\sum_{A \in \mathcal{S} \cap \Lambda, A \subset \Delta} \Phi_{A}^{a}(x) .
$$

Entonces

a) $\Phi_{\Lambda}^{a}$ es $\mathcal{F}_{\Lambda}$-medible, $\forall \Lambda \in \mathcal{S}$.

b) Para cada $\Lambda \in \mathcal{S}$ y $x \in E^{S}$ se tiene que:

$$
\begin{aligned}
& \text { (b1) Existe } \lim _{\Lambda \subset \Delta \uparrow S} H_{\Lambda, \Delta}^{\Phi^{a}}(x) \mathrm{y} \\
& \text { (b2) } H_{\Lambda}^{\Phi^{a}}(x):=\lim _{\Lambda \subset \Delta \uparrow S} H_{\Lambda, \Delta}^{\Phi^{a}}(x)=H_{\Lambda}^{\Phi}(x)-H_{\Lambda}^{\Phi}\left(a_{\Lambda} x_{S \backslash \Lambda}\right) .
\end{aligned}
$$

Luego $\Phi^{a}=\left(\Phi_{\Lambda}^{a}\right)_{\Lambda \in \mathcal{S}}$ es un potencial cuyo hamiltoniano satisface (b2).

c) $\Phi^{a}$ es $\lambda$-admisible.

d) $\Phi \sim \Phi^{a}$ (esto es: $H_{\Lambda}^{\Phi}-H_{\Lambda}^{\Phi^{a}}$ es $\mathcal{F}_{S \backslash \Lambda}$-medible $\left.\forall \Lambda \in \mathcal{S}\right)$.

e) $\varrho_{\Lambda, \lambda}^{\Phi}=\varrho_{\Lambda, \lambda}^{\Phi^{a}} \forall \Lambda \in \mathcal{S}$.

f) $\gamma_{\Lambda, \lambda}^{\Phi}(A \mid x)=\gamma_{\Lambda, \lambda}^{\Phi^{a}}(A \mid x) \Lambda \in \mathcal{S}, A \in \mathcal{F}, x \in E^{S}$.

g) $\mathcal{G}\left(\gamma_{\lambda}^{\Phi}\right)=\mathcal{G}\left(\gamma_{\lambda}^{\Phi^{a}}\right)$.

\section{Demostración.}

Parte a) Sigue de a) de la Proposición 3.3.23.

Parte b) Supongamos probada la siguiente

Afirmación 1: $H_{\Lambda, \Delta}^{\Phi^{a}}(x)=H_{\Lambda}^{\Phi}\left(x_{\Delta} a_{S \backslash \Delta}\right)-H_{\Lambda}^{\Phi}\left(a_{\Lambda} x_{\Delta \backslash \Lambda} a_{S \backslash \Delta}\right)$

De aquí, por la Proposición 3.3.25 se tiene que

$$
H_{\Lambda, \Delta}^{\Phi^{a}}(x)=\ln \left(\varrho_{\Lambda}^{\Phi}\right)\left(a_{\Lambda} x_{\Delta \backslash \Lambda} a_{S \backslash \Delta}\right)-\ln \left(\varrho_{\Lambda}^{\Phi}\right)\left(x_{\Delta} a_{S \backslash \Delta}\right) .
$$


Por (0) y la continuidad de ln se tiene que

$$
\lim _{\Lambda \subset \Delta \uparrow S} H_{\Lambda, \Delta}^{\Phi^{a}}(x)=\ln \left(\varrho_{\Lambda}^{\Phi}\right)\left(a_{\Lambda} x_{S \backslash \Lambda}\right)-\ln \left(\varrho_{\Lambda}^{\Phi}\right)(x)=(1) .
$$

De aquí y por la Proposición 3.3.25 se tiene que

$$
(1)=H_{\Lambda}^{\Phi}(x)-H_{\Lambda}^{\Phi}\left(a_{\Lambda} x_{S \backslash \Lambda}\right),
$$

lo cual prueba b).

Dem. de la Afirmación 1.

$$
\begin{aligned}
& H_{\Lambda, \Delta}^{\Phi}(x)=\sum_{A \subset \Delta} \Phi_{A}^{a}(x)-\sum_{A \subset(\Delta \backslash \Lambda)} \Phi_{A}^{a}(x)= \\
& =-\sum_{A \subset \Delta} p_{(a, A)}\left(\ln \left(\varrho_{A}^{\Phi}\right)\right)(x)+\sum_{A \subset(\Delta \backslash \Lambda)} p_{(a, A)}\left(\ln \left(\varrho_{A}^{\Phi}\right)\right)(x) \stackrel{b) \text { Prop. }{ }^{3.3 .25}}{=} p_{(a, A)}\left(\ln \left(\varrho_{\Delta}^{\Phi}\right)\right)(x)= \\
& =-\sum_{A \subset \Delta} p_{(a, A)}\left(\ln \left(\varrho_{\Delta}^{\Phi}\right)\right)(x)+\sum_{A \subset(\Delta \backslash \Lambda)}{ }^{\text {Prop. }}={ }^{3.3 .23}-\sum_{A \subset \Delta} p_{(a, A)}\left(\ln \left(\varrho_{\Delta}^{\Phi}\right)\right)\left(x_{\Delta} a_{S \backslash \Delta}\right)+ \\
& \sum_{A \subset(\Delta \backslash \Lambda)} p_{(a, A)}\left(\ln \left(\varrho_{\Delta}^{\Phi}\right)\right)\left(x_{\Delta \backslash \Lambda} a_{S \backslash(\Delta \backslash \Lambda)}\right) \\
& M \stackrel{\text { öbius }}{=}-\ln \left(\varrho_{\Delta}^{\Phi}\right)\left(x_{\Delta} a_{S \backslash \Delta}\right)+\ln \left(\varrho_{\Delta}^{\Phi}\right)\left(x_{\Delta \backslash \Lambda} a_{S \backslash(\Delta \backslash \Lambda)}\right) \\
& =-\ln \left(\varrho_{\Delta}^{\Phi}\right)(y)+\ln \left(\varrho_{\Delta}^{\Phi}\right)\left(a_{\Lambda} y_{S \backslash \Lambda}\right)=(2)
\end{aligned}
$$

con

$$
y=x_{\Delta} a_{S \backslash \Delta} .
$$

Por a) de la Proposición 3.3.25 tenemos:

$(2)=H_{\Delta}^{\Phi}(y)-H_{\Delta}^{\Phi}\left(a_{\Lambda} y_{S \backslash \Lambda}\right)=H_{\Delta}^{\Phi}\left(x_{\Delta} a_{S \backslash \Delta}\right)-H_{\Delta}^{\Phi}\left(a_{\Lambda} x_{\Delta} a_{S \backslash \Delta}\right)$.

$\underline{\text { Parte c) }}$ Por (b2):

$$
(3)=\exp \left(-H_{\Lambda}^{\Phi^{a}}(x)\right)=\exp \left(-H_{\Lambda}^{\Phi}(x)\right) \cdot \exp \left(H_{\Lambda}^{\Phi}\left(a_{\Lambda} x_{S \backslash \Lambda}\right)\right) .
$$

Luego:

$$
\begin{aligned}
& \int_{E^{\Lambda}} \exp \left(-H_{\Lambda}^{\Phi^{a}}\left(\xi x_{S \backslash \Lambda}\right)\right) \lambda^{\Lambda}(d \xi)=\exp \left(H_{\Lambda}^{\Phi}\left(a_{\Lambda} x_{S \backslash \Lambda}\right)\right) \\
& \times \int_{E^{\Lambda}} \exp \left(-H_{\Lambda}^{\Phi}\left(\xi x_{S \backslash \Lambda}\right)\right) \lambda^{\Lambda}(d \xi)<\infty .
\end{aligned}
$$


Parte d) Es inmediata por (b2).

Parte e) Sigue de (3) en la prueba de la Parte c).

Parte f) Es inmediata por la Parte e).

Parte g) $\mu \in \mathcal{G}\left(\gamma_{\lambda}^{\Phi}\right) \Leftrightarrow \gamma_{\Lambda}^{\Phi} \mu=\mu \forall \Lambda \in \mathcal{S} \stackrel{\text { Por }}{\Leftrightarrow}{ }^{f)} \gamma_{\Lambda}^{\Phi^{a}} \mu=\mu \forall \Lambda \in \mathcal{S} \Leftrightarrow \mu \in$ $\mathcal{G}\left(\gamma_{\lambda}^{\Phi^{a}}\right)$

Nota 3.3.30. Por la Proposición 3.3.21 se tiene que si $\Psi$ es otro potencial a-normalizado ( $a \in E$ como en el Teorema 3.3.29) tal que $\Psi \sim \Phi$, entonces $\Psi_{\Lambda}=\Phi_{\Lambda}^{a} \forall \Lambda \in \mathcal{S}$.

Proposición 3.3.31. Consideremos el Teorema 3.3.29.

Entonces: $\forall \Lambda \in \mathcal{S}$ y $\forall x \in E^{S}$ :

$$
\begin{aligned}
\Phi_{\Lambda}^{a}(x) & =\sum_{C \subset \Lambda}(-1)^{\#(\Lambda \backslash C)} H_{\Lambda}^{\Phi}\left(x_{C} a_{S \backslash C}\right) \\
& =\sum_{\Lambda \subset \Delta \in \mathcal{S}} \sum_{C \subset \Lambda}(-1)^{\#(\Lambda \backslash C)} \Phi_{\Delta}\left(x_{C} a_{S \backslash C}\right) .
\end{aligned}
$$

Demostración. Ejercicio. (Tener en cuenta que $\Phi_{\Delta}$ es $\mathcal{F}_{\Delta}$-medible $\forall \Delta \in$ $\mathcal{S}$ y c) de la Proposición 3.3.23).

Lema 3.3.32. Una condición suficiente para (0) del Teorema 3.3.29. Sea $f: E^{S} \longmapsto \mathbb{R} \mathcal{F}$-medible. Si $f$ es quasilocal, entonces

$$
O_{\infty}(f)(x)=0 \quad \forall x \in E^{S} .
$$

Demostración. Sea $\varepsilon>0$. Como $f$ es quasilocal, $\exists \Lambda \in \mathcal{S}$ y $f_{\Lambda}: E^{S} \longmapsto \mathbb{R}$ $\mathcal{F}_{\Lambda}$-medible, tal que:

$$
\left\|f-f_{\Lambda}\right\|_{\infty}<\frac{\varepsilon}{2} \Leftrightarrow \sup _{x}\left|f(x)-f_{\Lambda}(x)\right|<\frac{\varepsilon}{2} .
$$

Sea $\xi \in E^{S \backslash \Lambda}$ cualquiera. Como $f_{\Lambda}$ es $\mathcal{F}_{\Lambda}$-medible.se tiene que:

$$
\begin{array}{cl}
\forall x \in E^{S} \quad & \left|f(x)-f\left(x_{\Lambda} \xi\right)\right| \leq\left|f\left(x_{\Lambda} \xi\right)-f_{\Lambda}\left(x_{\Lambda} \xi\right)\right|+ \\
& \left|f(x)-f_{\Lambda}\left(x_{\Lambda} x_{S \backslash \Lambda}\right)\right|<\frac{\varepsilon}{2}+\frac{\varepsilon}{2}=\varepsilon .
\end{array}
$$

\subsubsection{El potencial $\Phi^{a}$ para distintos ejemplos}

Ejemplo 3.3.33 (Continuación del Ejemplo 3.2.3). Sea $\beta \in \mathbb{R}$. Sea

$$
\begin{aligned}
\Phi_{\{s, t\}}(x) & =\beta x(s) x(t) \quad \text { si } t \in V_{s}^{1} \\
\Phi_{\Lambda} & \equiv 0 \quad \text { en todo otro caso. }
\end{aligned}
$$


Entonces para $a=-1$ se tiene:

$$
\begin{aligned}
\Phi_{\{s\}}^{a}(x) & =8 \beta \quad \text { si } x(s)=1 \\
\Phi_{\{s, t\}}^{a}(x) & =4 \beta \quad \text { si } t \in V_{s}^{1}, x(s)=x(t)=1 \\
\Phi_{\Lambda}^{a} & \equiv 0 \quad \text { para cualquier otro } \Lambda \in \mathcal{S} .
\end{aligned}
$$

Ejemplo 3.3.34 (Continuación del Ejemplo 3.2.3). Sean $\alpha$ y $\beta \in \mathbb{R}$

$$
\begin{aligned}
\Phi_{\{s\}}(x) & =\alpha x(s) \\
\Phi_{\{s, t\}}(x) & =\beta x(s) x(t) \quad \text { si } t \in V_{s}^{1} \\
\Phi_{\Lambda} & \equiv 0 \text { en todo otro caso. }
\end{aligned}
$$

Entonces, para $a=-1$ se tiene:

$$
\begin{aligned}
\Phi_{\{s\}}^{a}(x) & =-2 \alpha+8 \beta \quad \text { si } x(s)=1 \\
\Phi_{\{s, t\}}^{a}(x) & =4 \beta \quad \text { si } t \in V_{s}^{1}, x(s)=x(t)=1 \\
\Phi_{\Lambda}^{a} & \equiv 0 \quad \text { para cualquier otro } \Lambda \in \mathcal{S} .
\end{aligned}
$$

Ejemplo 3.3.35 (Continuación del Ejemplo 3.2.4). Sean $E=\{0,1\} ; \beta \in$ $\mathbb{R}$.

$$
\begin{aligned}
\Phi_{\{s, t\}}(x) & =\beta x(s) x(t) \quad \text { si } t \in V_{s}^{1} \\
\Phi_{\Lambda} & \equiv 0 \text { en todo otro caso. }
\end{aligned}
$$

Si $a=0$, evidentemente $\Phi$ está a-normalizado.

Sea $a=1$. Entonces

$$
\begin{aligned}
\Phi_{\{s\}}^{a}(x) & =4 \beta \quad \text { si } x(s)=0 \\
\Phi_{\{s, t\}}^{a}(x) & =2 \beta \quad \text { si } t \in V_{s}^{1}, x(s)=x(t)=0 \\
\Phi_{\Lambda}^{a} & \equiv 0 \quad \text { para cualquier otro caso. }
\end{aligned}
$$

Ejemplo 3.3.36 (Continuación del Ejemplo 3.2.6). Sea $E=\{-1,1\}$.

Sean: $s=\left(s_{1}, s_{2}\right) ; t_{ \pm 1}=\left(s_{1} \pm 1, s_{2}\right) ; t_{ \pm 2}=\left(s_{1}, s_{2} \pm 1\right)$;

$$
\begin{aligned}
& \Phi_{\{s, t\}}(x)=\beta_{H} x(s) x(t) \quad \text { si } t=t_{+1} \text { ó } t=t_{-1} \\
& \Phi_{\{s, t\}}(x)=\beta_{V} x(s) x(t) \quad \text { si } t=t_{+2} \text { ó } t=t_{-2} .
\end{aligned}
$$

Sea $a=-1$. Entonces:

$$
\begin{aligned}
\Phi_{\{s\}}^{a}(x) & =4\left(\beta_{H}+\beta_{V}\right) \quad \text { si } x(s)=1 \\
\Phi_{\{s, t\}}^{a}(x) & =4 \beta_{H} \quad \text { si } t=t_{+1} \text { ó } t=t_{-1}, x(s)=x(t)=1 \\
\Phi_{\{s, t\}}^{a}(x) & =4 \beta_{V} \quad \text { si } t=t_{+2} \text { ó } t=t_{-2}, x(s)=x(t)=1
\end{aligned}
$$




\subsection{Potenciales invariante por traslaciones}

Para cada $t \in \mathbb{Z}^{2}$ sea $\theta_{t}: E^{\mathbb{Z}^{2}} \mapsto E^{\mathbb{Z}^{2}}$ definida por:

$$
\theta_{t}(x)(s)=x(s-t)
$$

Definición 3.4.1. Sea $\Phi=\left(\Phi_{\Lambda}\right)_{\Lambda \in \mathcal{S}}$ un potencial. Se dice que $\Phi$ es invariante por traslaciones si

$$
\Lambda \in \mathcal{S}, t \in S \Rightarrow \Phi_{\Lambda+t}(x)=\Phi_{\Lambda}\left(\theta_{-t}(x)\right), x \in E^{S} .
$$

Ejemplo 3.4.2. Potencial invariante por traslaciones asociado a un $V \in$ $\mathcal{S}$ y $\phi_{V}: E^{V} \mapsto \mathbb{R} \mathcal{E}^{V}$-medible y acotada.

Sea $\mathcal{S}_{V}:=\left\{\Lambda \subset S / \Lambda=V+t\right.$, para $\left.t \in \mathbb{Z}^{2}\right\}$.

Como $V$ es finito:

$$
V+t \neq V+s \quad \text { si } s \neq t
$$

Sea

$$
\Phi_{V}:=\phi_{V} \circ \sigma_{V} .
$$

Para cada $V \in \mathcal{S}$ sea $\Phi_{\Lambda}: E^{S} \longmapsto \mathbb{R}$ dada por:

$$
\Phi_{\Lambda}(x)= \begin{cases}0 \quad \forall x \in E^{S} & \text { si } \Lambda \notin \mathcal{S}_{V} \\ \Phi_{V}\left(\theta_{-t}(x)\right) & \forall x \in E^{S}, \Lambda=V+t .\end{cases}
$$

Por (3.4) esta definición no presenta ambigüedades.

Afirmación 1: $\Phi_{\Lambda}$ es $\mathcal{F}_{\Lambda}$-medible, $\forall \Lambda \in \mathcal{S}$.

Demostración. Ejercicio.

Como $\Lambda$ y $V$ son finitos, es fácil ver que

$$
\#\left(\mathcal{S}_{V} \cap \Lambda\right)<\infty .
$$

Afirmación 2: Para todo $\Lambda \in \mathcal{S}$ está definido:

$$
H_{\Lambda}^{\Phi}:=\sum_{\Delta \in \mathcal{S} \cap \mathcal{V}} \Phi_{\Delta}=\sum_{t \in \Lambda-V} \Phi_{V} \circ \theta_{-t} .
$$

Demostración. Ejercicio.

Ejemplo 3.4.3. Potencial invariante por traslaciones asociado a $p \geq 2$ conjuntos $V_{1}, \ldots, V_{p}$ en $\mathcal{S}$ y funciones $\phi_{k}: E^{V_{k}} \mapsto \mathbb{R}$ para $k=1, \ldots, p$ $\mathcal{E}^{k}$-medibles.

Sea

$$
\mathcal{S}\left(V_{1}, \ldots, V_{k}\right):=\left\{V_{k}+t / t \in \mathbb{Z}^{2}, k=1, \ldots, p\right\} .
$$


Notemos que como $V_{k}$ es finito:

$$
V_{k}+s \neq V_{k}+t \quad \text { si } s \neq t \text {, }
$$

cualquiera sea $1 \leq k \leq p$.

Sea $\theta=\left(\theta_{1}, \ldots, \theta_{p}\right)^{\prime} \in \mathbb{R}^{p}$. Para cada $t \in \mathbb{Z}^{2}$ y cada $k=1, \ldots, p$ sea $\Phi_{V_{k}+t}: E^{S} \mapsto \mathbb{R}$ dada por

$$
\Phi_{V_{k}+t}(x)=\phi_{k}\left(\sigma_{V_{k}}\left(\theta_{-t}(x)\right)\right) .
$$

Sea

$$
\Phi_{\Lambda} \equiv 0 \quad \text { si } \Lambda \notin \mathcal{S}\left(V_{1}, \ldots, V_{k}\right) \text {. }
$$

Afirmación 1: Si $\Lambda \in \mathcal{S}$, entonces $\Phi_{\Lambda}$ es $\mathcal{F}_{\Lambda}$-medible.

Demostración. Ejercicio.

Afirmación 2: Para todo $\Lambda \in \mathcal{S}$ está definido:

$$
H_{\Lambda}^{\Phi}(x):=\sum_{k=1}^{p} \theta_{k} \sum_{t \in \Lambda-V_{k}} \Phi_{t+V_{k}}(x) .
$$

Demostración. Ejercicio.

\subsection{Auto-modelos de Besag}

Salvo expresa mención encontrario suponemos estar en la situación expuesta al inicio de este Capítulo.

Definición 3.5.1. Sea $\Phi=\left(\Phi_{\Lambda}\right)_{\Lambda \in \mathcal{S}}$ un potencial sobre $\left(E^{S}, \mathcal{F}\right)$. Diremos que $\Phi$ es un potencial sobre pares de $S$ si $\Phi_{\Lambda} \equiv 0 \forall \Lambda \in \mathcal{S}$ con $\#(\Lambda) \geq 3$.

Ejemplo 3.5.2 (Automodelo de Besag generalizado). Caso particular de un potencial sobre pares de $S$ en el marco del Ejemplo 3.2.8.

Sea:

$$
\begin{aligned}
J & : \quad S \times S \mapsto \mathbb{R} \text { simétrica; } \\
B_{s} & : \quad E \mapsto \mathbb{R}, C_{s}: E \mapsto \mathbb{R} \text {-medibles, } s \in S ; \\
\mathcal{C} & : \quad=\{\Lambda \in \mathcal{S} / \#(\Lambda) \leq 2\} .
\end{aligned}
$$

Para cada $C \in \mathcal{S}:$

$$
\begin{aligned}
& p_{C}=2 \quad \text { si } \#(C)=1, p_{C}=1 \quad \text { si } \#(C)=2 ; \\
& \theta_{C}=\left\{\begin{array}{c}
(J(s, s), 1)^{\prime} \quad \text { si } C=\{s\}, s \in S ; \\
J(s, t) \quad \text { si } C=\{s, t\}, s \neq t \text { ambos en } S ;
\end{array}\right.
\end{aligned}
$$


$\phi_{C}: E^{S} \mapsto \mathbb{R}^{p_{C}}$ dada por:

$$
\phi_{C}(x)=\left\{\begin{array}{l}
\left(B_{s}(x(s)), C_{s}(x(s))\right) \text { si } C=\{s\}, s \in S \\
B_{s}(x(s)) B_{t}(x(t)) \text { si } C=\{s, t\}, s \neq t .
\end{array}\right.
$$

Para cada $\Lambda \in \mathcal{S}$ definimos $\Phi_{\Lambda}: E^{S} \mapsto \mathbb{R}$ por:

$$
\begin{aligned}
\Phi_{\Lambda} & \equiv 0 \text { si } \Lambda \notin \mathcal{C} . \\
\Phi_{\{s\}}(x) & =\theta_{\{s\}}^{\prime} \phi_{\{s\}}(x)=J(s, s) B_{s}(x(s))+C_{s}(x(s)) \\
\Phi_{\{s, t\}}(x) & =\theta_{\{s, t\}}^{\prime} \phi_{\{s, t\}}(x)=J(s, t) B_{s}(x(s)) B_{t}(x(t)) \quad \text { si } s \neq t .
\end{aligned}
$$

Notemos que para que $\Phi=\left(\Phi_{\Lambda}\right)_{\Lambda \in \mathcal{S}}$ sea un potencial (de acuerdo a la Definición 3.1.1) debe cumplirse que existe

$$
\sum_{\Delta \in \mathcal{S} \cap \Lambda} \Phi_{\Delta}, \quad \forall \Lambda \in \mathcal{S} .
$$

En este caso, se puede ver que es suficiente asumir

$$
\sum_{t \in S}\left|J(s, t) B_{t}(x(t))\right|<\infty, \quad \forall s \in S .
$$

También, si $\lambda$ es una medida $\sigma$-finita sobre $(E, \mathcal{E})$, para que $\Phi$ sea $\lambda$ admisible debe cumplirse

$$
\int_{E^{\Lambda}} \exp \left(-\sum_{\Delta \in \mathcal{S} \cap \Lambda} \Phi_{\Delta}\left(\xi x_{S \backslash \Lambda}\right)\right) \lambda^{\Lambda}(d \xi)<\infty
$$

cualquiera sea $x \in E^{S}$ y $\Lambda \in \mathcal{S}$.

Teorema 3.5.3. Sean: $\lambda$ una medida $\sigma$-finita; $E$ (con más de dos puntos) un e.m. separable y completo; $\Phi=\left(\Phi_{\Lambda}\right)_{\Lambda \in \mathcal{S}}$ un potencial sobre $\left(E^{S}, \mathcal{F}\right)$ $a$-normalizado con $a \in E, \lambda$-admisible y sobre pares de $S$.

a) Supongamos que para todo $s \in S$ y todo $x \in E^{S}$ se cumple:

$$
\ln \left(\varrho_{\{s\}, \lambda}^{\Phi}(x)\right)=a_{s}(x) b_{s}(x)-c_{s}(x)-d_{s}(x)
$$

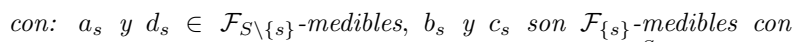
$b_{s}\left(a x_{S \backslash\{s\}}\right)=c_{s}\left(a x_{S \backslash\{s\}}\right)=0$ cualquiera sea $x \in E^{S}$, no idénticamente nulas.

Entonces existe $J: S \times S \mapsto \mathbb{R}$ simétrica tal que:

$$
\text { a1) } a_{s}(x)=-J(s, s)-\sum_{t \neq s} J(s, t) b_{t}(x), \quad x \in E^{S}, s \in S .
$$


a2) $\Phi_{\{s\}}(x)=J(s, s) b_{s}(x)+c_{s}(x), \quad x \in E^{S}, s \in S$.

a3) $\Phi_{\{s, t\}}(x)=J(s, t) b_{s}(x) b_{t}(x), \quad x \in E^{S}, s \in S, t \in S, s \neq t$.

b) Recíprocramente, supongamos que existen $J: S \times S \mapsto \mathbb{R}$, simétrica, $b_{s}: E^{S} \mapsto \mathbb{R}, c_{s}: E^{S} \mapsto \mathbb{R} \mathcal{F}_{\{s\}}$-medibles, $\forall s \in S$ con $b_{s}\left(a x_{S \backslash\{s\}}\right)=$ $c_{s}\left(a x_{S \backslash\{s\}}\right)=0, x \in E^{S}, s \in S$ y satisfacen a1), a2) y a3). Entonces para todo $s \in S$ y todo $x \in E^{S}$ se cumple:

$$
\ln \left(\varrho_{\{s\}, \lambda}^{\Phi}(x)\right)=a_{s}(x) b_{s}(x)-c_{s}(x)-d_{s}(x)
$$

con $d_{s} \in \mathcal{F}_{S \backslash\{s\}}$-medible.

\section{Demostración. Parte b).}

Para todo $s \in S$ y todo $x \in E^{S}$ tenemos:

$$
\begin{aligned}
\varrho_{\{s\}, \lambda}^{\Phi}(x) & =\frac{\exp \left(-H_{\{s\}}^{\Phi}(x)\right)}{Z_{\{s\}, \lambda}^{\Phi}(x)} \\
\varrho_{\{s\}, \lambda}^{\Phi}\left(a x_{S \backslash\{s\}}\right) & =\frac{\exp \left(-H_{\{s\}}^{\Phi}\left(a x_{S \backslash\{s\}}\right)\right)}{Z_{\{s\}, \lambda}^{\Phi}\left(a x_{S \backslash\{s\}}\right)} .
\end{aligned}
$$

De aquí, como $\Phi$ es $a$-normalizado: $H_{\{s\}}^{\Phi}\left(a x_{S \backslash\{s\}}\right)=0$ y como $Z_{\{s\}, \lambda}^{\Phi}$ es $\mathcal{F}_{S \backslash\{s\}}$-medible, resulta:

$$
\frac{\varrho_{\{s\}, \lambda}^{\Phi}(x)}{\varrho_{\{s\}, \lambda}^{\Phi}\left(a X_{S \backslash\{s\}}\right)}=\exp \left(-H_{\{s\}}^{\Phi}(x)\right) .
$$

Luego:

$$
\ln \left(\varrho_{\{s\}, \lambda}^{\Phi}(x)\right)=-\Phi_{\{s\}}(x)-\sum_{t \neq s} \Phi_{\{s, t\}}(x)-d_{s}(x)=(1)
$$

con

$$
d_{s}(x):=-\ln \left(\varrho_{\{s\}, \lambda}^{\Phi}\left(a x_{S \backslash\{s\}}\right)\right),
$$

que es por lo tanto $\mathcal{F}_{S \backslash\{s\} \text {-medible. }}$

Por lo supuesto:

$$
\begin{aligned}
(1) & =-J(s, s) b_{s}(x)-c_{s}(x)-\sum_{s \neq t} J(s, t) b_{t}(x) b_{s}(x)-d_{s}(x) \\
& =\left(-J(s, s)-\sum_{s \neq t} J(s, t) b_{t}(x)\right) b_{s}(x)-c_{s}(x)-d_{s}(x) \\
& =a_{s}(x) b_{s}(x)-c_{s}(x)-d_{s}(x) .
\end{aligned}
$$

La parte b) está probada. 


\section{Parte a)}

Como vimos al probar b), se tiene, $\forall s \in S$ y $\forall x \in E^{S}$ :

$$
\ln \left(\varrho_{\{s\}, \lambda}^{\Phi}(x)\right)=-\Phi_{\{s\}}(x)-\sum_{t \neq s} \Phi_{\{s, t\}}(x)+\ln \left(\varrho_{\{s\}, \lambda}^{\Phi}\left(a X_{S \backslash\{s\}}\right)\right) .
$$

Por lo supuesto en a) y por ser $d_{s} \mathcal{F}_{S \backslash\{s\}}$-medible

$$
\Phi_{\{s\}}(x)+\sum_{t \neq s} \Phi_{\{s, t\}}(x)=-a_{s}(x) b_{s}(x)+c_{s}(x) .
$$

De aquí se deduce, dado que $\Phi$ es $a$-normalizada,

$$
\Phi_{\{s\}}(x)=\Phi_{\{s\}}\left(x(s) a_{S \backslash\{s\}}\right)=-a_{s}(\tilde{a}) b_{s}(x)+c_{s}(x) .
$$

Sean $r \in S, s \in S, r \neq s y \in E^{S}$ tal que

$$
\begin{aligned}
& y(s)=x(s), y(r)=x(r), y(t)=a \forall t \notin\{s, r\} \\
& \Phi_{\{s\}}(y)+\sum_{t \neq s} \Phi_{\{s, t\}}(y)= \Phi_{\{s\}}(y)+\Phi_{\{s, r\}}(y) \\
&=\Phi_{\{s\}}(x)+\Phi_{\{s, r\}}(x) .
\end{aligned}
$$

Por (2) tenemos entonces:

$$
\Phi_{\{s\}}(x)+\Phi_{\{s, r\}}(x)=-a_{s}\left(x(r) a_{S \backslash\{r\}}\right) b_{s}(x)+c_{s}(x) .
$$

De (3) y (4) resulta entonces:

$$
\Phi_{\{s, r\}}(x)=\left(a_{s}(\tilde{a})-a_{s}\left(x(r) a_{S \backslash\{r\}}\right)\right) b_{s}(x),
$$

también, intercambiando los roles de $s$ y $r$ :

$$
\Phi_{\{s, r\}}(x)=\left(a_{r}(\tilde{a})-a_{r}\left(x(s) a_{S \backslash\{s\}}\right)\right) b_{r}(x) .
$$

Luego, $\xi \in E$ y $\eta \in E$ :

$$
\begin{gathered}
\left(a_{s}(\tilde{a})-a_{s}\left(\xi a_{S \backslash\{r\}}\right)\right) b_{s}\left(\eta x_{S \backslash\{s\}}\right)= \\
\left(a_{r}(\tilde{a})-a_{r}\left(\eta a_{S \backslash\{s\}}\right)\right) b_{r}\left(\xi x_{S \backslash\{r\}}\right) .
\end{gathered}
$$

Sean: $E_{t}:=\left\{\zeta \in E / b_{t}\left(\zeta x_{S \backslash\{t\}}\right) \neq 0\right\} . \quad \forall t \in S$. Como suponemos $E_{t} \neq \emptyset \forall t \in S$ se tiene:

$\xi \in E_{r}$ y $\eta \in E_{s} \Rightarrow$

$$
\begin{aligned}
& \left(a_{s}(\tilde{a})-a_{s}\left(\xi a_{S \backslash\{r\}}\right)\right)\left(b_{r}\left(\xi x_{S \backslash\{r\}}\right)\right)^{-1} \\
= & \left(a_{r}(\tilde{a})-a_{r}\left(\eta a_{S \backslash\{s\}}\right)\right)\left(b_{s}\left(\eta x_{S \backslash\{s\}}\right)\right)^{-1} .
\end{aligned}
$$


Luego $\xi \mapsto\left(a_{s}(\tilde{a})-a_{s}\left(\xi a_{S \backslash\{r\}}\right)\right)\left(b_{r}\left(\xi x_{S \backslash\{r\}}\right)\right)^{-1}$ es constante y la denotamos por $J(r, s)$.

Análogamente $\eta \mapsto\left(a_{r}(\tilde{a})-a_{r}\left(\eta a_{S \backslash\{s\}}\right)\right)\left(b_{s}\left(\eta x_{S \backslash\{s\}}\right)\right)^{-1}$ es constante y la denotamos con $J(s, r)$.

Por (6) tiene entonces

$$
J(s, r)=J(r, s) .
$$

Luego por (5):

$$
\Phi_{\{s, r\}}=J(s, r) b_{r}(x) b_{s}(x) .
$$

Por (2) tenemos entonces:

$$
\begin{aligned}
& -a_{s}(x) b_{s}(x)+c_{s}(x)=\Phi_{\{s\}}(x)+\sum_{s \neq t} \Phi_{\{s, t\}}(x) \\
& =-a_{s}(\tilde{a}) b_{s}(x)+c_{s}(x)+\sum_{s \neq t} J(s, t) b_{s}(x) b_{t}(x) .
\end{aligned}
$$

Luego tomando $x \operatorname{con} x(s) \in E_{s}$ :

$$
a_{s}(x)=a_{s}(\widetilde{a})-\sum_{s \neq t} J(s, t) b_{t}(x) .
$$

Tomando entonces $J(s, s)=-a_{s}(\widetilde{a})$, resulta:

$$
\begin{aligned}
a_{s}(x) & =-J(s, s)-\sum_{s \neq t} J(s, t) b_{t}(x) \\
\Phi_{\{s\}}(x) & =J(s, s) b_{s}(x)+c_{s}(x)
\end{aligned}
$$

y

$$
\Phi_{\{s, t\}}(x)=J(s, t) b_{s}(x) b_{t}(x) \quad s \neq t .
$$

\subsection{Ejemplos de auto-modelos $\mathcal{G}$-markovianos}

En esta Sección consideraremos:

$S \subset \mathbb{Z}^{2} ; \mathcal{V}:=\left\{V_{s} / s \in S\right\}$ un sistema de vecindades sobre $S ; \lambda$ una medida $\sigma$-finita sobre $(E, \mathcal{E})$;

$\mathcal{G}=(S, \mathcal{V}) ; \mathcal{C}(\mathcal{G})=\{C \in \mathcal{S} / C$ es $\mathcal{G}$-completo $\}$ (esto es: $\mathcal{C}(\mathcal{G})=$ $\left\{C \in \mathcal{S} / \#(C)=1\right.$ os $\left.\left.s \in C, t \in C \Rightarrow t \in V_{s}\right\}\right)$.

$\Phi=\left(\Phi_{\Lambda}\right)_{\Lambda \in \mathcal{S}}$ un potencial sobre $\left(E^{S}, \mathcal{F}\right) \lambda$-admisible tal que

$$
\Phi_{\Lambda}=0 \quad \forall \Lambda \notin \mathcal{C}(\mathcal{G})
$$

esto es, $\Phi$ es un $\mathcal{G}$-potencial. 
Si $E$ es finito consideraremos siempre $\mathcal{E}=\mathcal{P}(E)$ y $\lambda$ la medida de conteo.

Dado $s \in S$ definimos $\gamma_{s, \lambda}^{\Phi, 0}: \mathcal{E} \times E^{S} \mapsto[0,1]$ por

$$
\begin{aligned}
\gamma_{s, \lambda}^{\Phi, 0}(A \mid x) & =\gamma_{\{s\}, \lambda}^{\Phi}\left(\sigma_{s}^{-1}(A) \mid x\right) \\
& =\int_{A} \varrho_{\{s\}, \lambda}^{\Phi}\left(\xi x_{S \backslash\{s\}}\right) \lambda(d \xi) .
\end{aligned}
$$

Luego $\xi \mapsto \varrho_{\{s\}, \lambda}^{\Phi}\left(\xi x_{S \backslash\{s\}}\right)$ es una $\lambda$-densidad de la probabilidad sobre $(E, \mathcal{E})$ dada por $\gamma_{s, \lambda}^{\Phi, 0}(\cdot \mid x)$.

Ejemplo 3.6.1 (Automodelo logístico para $E=\{0,1\}$ ). Sean: $J: S \times S \mapsto \mathbb{R}$ simétrica tal que:

$$
J(s, t)=0 \text { si } t \notin V_{s} .
$$

Para cada $s \in S$ sea $b_{s}: E^{S} \mapsto \mathbb{R}$ dada por:

$$
b_{s}(x)=x(s),
$$

luego:

$$
b_{s}(x)=\left\{\begin{array}{cl}
0 & \text { si } x=\left(0 x_{S \backslash\{s\}}\right) \\
1 & \text { si } x=\left(1 x_{S \backslash\{s\}}\right)
\end{array}\right.
$$

$c_{s}: E^{S} \mapsto \mathbb{R}$ dada por: $c_{s}(x)=0 \forall x \in E^{S}$.

$$
\ln \left(\varrho_{\{s\}, \lambda}^{\Phi}(x)\right)=a_{s}(x) b_{s}(x)-d_{s}(x),
$$

con

$$
\begin{aligned}
& a_{s}(x)=-J(s, s)-\sum_{t \in V_{s}} J(s, t) x(t) \\
& d_{s}(x)=-\ln \left(\varrho_{\{s\}, \lambda}^{\Phi}\left(0 x_{S \backslash\{s\}}\right)\right) .
\end{aligned}
$$

Luego, si

$$
p_{s, x}:=\gamma_{s, \lambda}^{\Phi, 0}(\{1\} \mid x),
$$

entonces

$$
\operatorname{logit}\left(p_{s, x}\right):=\ln \left(\frac{p_{s, x}}{1-p_{s, x}}\right)=a_{s}(x) .
$$

Ejemplo 3.6.2 (Automodelo binomial para $E=\{0,1, \ldots, N\}$ con $N \in \mathbb{N}$ ). Como es habitual, consideramos $E=\{0, \ldots, N\}, \lambda$ medida de conteo sobre $(E, \mathcal{P}(E))$.

Para cada $s \in S$ sea $\theta_{s}: E^{S} \mapsto(0,1)$ una función $\mathcal{F}_{S \backslash\{s\}}$-medible.

Nos preguntamos si es posible que

$$
\gamma_{s, \lambda}^{\Phi, 0}(\cdot \mid x) \sim \operatorname{Bi}\left(N, \theta_{s}(x)\right) .
$$


Para ello, debe cumplirse que

$$
\varrho_{\{s\}, \lambda}^{\Phi}\left(\xi x_{S \backslash\{s\}}\right)=\left(\begin{array}{c}
N \\
\xi
\end{array}\right) \theta_{s}(x)^{\xi}\left(1-\theta_{s}(x)\right)^{N-\xi}
$$

para todo $\xi \in E$.

Equivalentemente:

$$
\varrho_{\{s\}, \lambda}^{\Phi}\left(\xi x_{S \backslash\{s\}}\right)=\left(\begin{array}{c}
N \\
\xi
\end{array}\right)\left(\frac{\theta_{s}(x)}{1-\theta_{s}(x)}\right)^{\xi}\left(1-\theta_{s}(x)\right)^{N}
$$

para todo $\xi \in E$.

Luego, debe ser:

$$
\ln \left(\varrho_{\{s\}, \lambda}^{\Phi}\left(\xi X_{S \backslash\{s\}}\right)\right)=\xi \ln \left(\frac{\theta_{s}(x)}{1-\theta_{s}(x)}\right)+\ln \left(\left(\begin{array}{c}
N \\
\xi
\end{array}\right)\right)+N \ln \left(1-\theta_{s}(x)\right) .
$$

Luego podemos definir $a_{s}, b_{s}, c_{s} y d_{s}$ satisfaciendo a) del Teorema 3.5.3.

En efecto, basta definir:

$$
\begin{array}{ll}
a_{s}(x)=\ln \left(\frac{\theta_{s}(x)}{1-\theta_{s}(x)}\right), & b_{s}(x)=x(s), \\
c_{s}(x)=-\ln \left(\left(\begin{array}{c}
N \\
x(s)
\end{array}\right)\right), & d_{s}(x)=-N \ln \left(1-\theta_{s}(x)\right) .
\end{array}
$$

Luego por el Teorema 3.5.3 existe $J: S \times S \mapsto \mathbb{R}$ simétrica tal que

$$
a_{s}(x)=-J(s, s)-\sum_{t \in V_{s}} J(s, t) x(t) .
$$

Por otra parte, una cuenta directa prueba que

$$
\theta_{s}(x)=\left(1+e^{-a_{s}(x)}\right)^{-1} .
$$

Por el Teorema 3.5.3 tenemos entonces

$$
\begin{aligned}
\Phi_{\{s\}}(x) & =J(s, s) x(s)-\ln \left(\left(\begin{array}{c}
N \\
x(s)
\end{array}\right)\right), \quad y \\
\Phi_{\{s, t\}}(x) & =J(s, t) x(s) x(t), \quad \text { si } t \in V_{s} .
\end{aligned}
$$

Ejemplo 3.6.3 (Automodelo Poisson para $E=\mathbb{N} \cup\{0\}$ ). Consideremos sobre $(E, \mathcal{P}(E))$ la medida de conteo.

Para cada $s \in S$, sea $\lambda_{s}: E^{S} \mapsto[0,+\infty)$ una función $\mathcal{F}_{S \backslash\{s\}}$-medible.

Nos preguntamos si es posible que

$$
\gamma_{s, \lambda}^{\Phi, 0}(\cdot \mid x) \sim \operatorname{Poisson}\left(\lambda_{s}(x)\right) .
$$


Para ello, debe cumplirse que

$$
\varrho_{\{s\}, \lambda}^{\Phi}\left(\xi x_{S \backslash\{s\}}\right)=e^{-\lambda_{s}(x)} \frac{\left(\lambda_{s}(x)\right)^{\xi}}{\xi !} \quad \forall \xi x_{S \backslash\{s\}} \in E^{S} .
$$

Luego debe cumplirse:

$$
\ln \left(\varrho_{\{s\}, \lambda}^{\Phi}\left(\xi x_{S \backslash\{s\}}\right)\right)=-\lambda_{s}(x)+\xi \ln \left(\lambda_{s}(x)\right)-\ln (\xi !) .
$$

Definimos entonces:

$$
\begin{array}{ll}
a_{s}(x)=\ln \left(\lambda_{s}(x)\right), & b_{s}(x)=x(s), \\
c_{s}(x)=\ln (x(s) !), & d_{s}(x)=\lambda_{s}(x) .
\end{array}
$$

Por el Teorema 3.5.3 existe $J: S \times S \mapsto \mathbb{R}$ simétrica tal que:

$$
a_{s}(x)=-J(s, s)-\sum_{t \in V_{s}} J(s, t) x(t) .
$$

También:

$$
\begin{aligned}
\Phi_{\{s\}}(x) & =J(s, s) x(s)+\ln (x(s) !), \quad y \\
\Phi_{\{s, t\}}(x) & =J(s, t) x(s) x(t), \quad \text { si } t \in V_{s} .
\end{aligned}
$$

Afirmación: Por ser $\Phi \lambda$-admisible se tiene $J(s, t) \geq 0$ para $t \in V_{s}$.

Demostración. Por ser $\Phi \lambda$-admisible $\forall \Lambda \in \mathcal{S}$ debe cumplirse para todo $x \in E^{S}$

$$
\sum_{\xi \in E^{\Lambda}} \exp \left(-H_{\Lambda}^{\Phi}\left(\xi x_{S \backslash \Lambda}\right)\right)<\infty .
$$

Sea $s \in S$ y $t \in V_{s}$. Sea $\Lambda=\{s, t\}$.

Para cada $\xi \in \mathbb{N}$ considerando $x \in E^{S}$ con $x(s)=\xi=x(t)$ y $x(u)=0$ $\forall u \notin \Lambda$ tenemos por (1):

$$
\sum_{\xi=0}^{\infty} \exp \left(-\Phi_{\{s\}}\left(\xi \xi x_{S \backslash \Lambda}\right)-\Phi_{\{t\}}\left(\xi \xi x_{S \backslash \Lambda}\right)-\Phi_{\{s, t\}}\left(\xi \xi x_{S \backslash \Lambda}\right)\right)<\infty .
$$

Luego:

$$
\sum_{\xi=0}^{\infty} \exp \left(-J(s, s) \xi-J(t, t) \xi-2 \ln (\xi !)-J(s, t) \xi^{2}\right)<\infty .
$$

De aquí no es difícil ver que debe ser $J(s, t) \geq 0$. 
Ejemplo 3.6.4 (Automodelo exponencial para $E=[0,+\infty)$ ). Sea $\mathcal{E}$ la $\sigma$-álgebra de Borel (usual) de E. Sea $\lambda$ la medida de Lebesgue sobre $(E, \mathcal{E})$. Para cada $s \in S$ sea $\lambda_{s}: E^{S} \mapsto(0,+\infty)$ una función $\mathcal{F}_{S \backslash\{s\}}$-medible.

Veremos que es posible tener

$$
\gamma_{s, \lambda}^{\Phi, 0}(\cdot \mid x) \sim \operatorname{Exp}\left(\lambda_{s}(x)\right)
$$

Debemos tener:

$$
\varrho_{\{s\}, \lambda}^{\Phi}\left(\xi x_{S \backslash\{s\}}\right)=\lambda_{s}(x) e^{-\lambda_{s}(x) \xi} \quad \forall \xi x_{S \backslash\{s\}} \in E^{S} .
$$

Luego debe cumplirse:

$$
\ln \left(\varrho_{\{s\}, \lambda}^{\Phi}\left(\xi x_{S \backslash\{s\}}\right)\right)=-\lambda_{s}(x) \xi+\ln \left(\lambda_{s}(x)\right) .
$$

Para poder aplicar el Teorema 3.5.3 definimos para $s \in S$ y $x \in E^{S}$ :

$$
\begin{aligned}
& a_{s}(x)=-\lambda_{s}(x), \quad b_{s}(x)=x(s), \\
& c_{s}(x)=0, \quad d_{s}(x)=\ln \left(\lambda_{s}(x)\right) .
\end{aligned}
$$

Por el Teorema 3.5.3 existe $J: S \times S \mapsto \mathbb{R}$ simétrica tal que:

$$
a_{s}(x)=-J(s, s)-\sum_{t \in V_{s}} J(s, t) x(t) .
$$

También:

$$
\begin{aligned}
\Phi_{\{s\}}(x) & =J(s, s) x(s), \quad s \in S y x \in E^{S}, y \\
\Phi_{\{s, t\}}(x) & =J(s, t) x(s) x(t), \quad s \in S y x \in E^{S}, t \in V_{s} .
\end{aligned}
$$

Por ser $\Phi \lambda$-admisible se puede ver que debe ser $J(s, s)>0$ y $J(s, t) \geq 0$ para $s \in S, y t \in V_{s}$. 


\section{Capítulo 4}

\section{Inferencias en Modelos Espaciales}

\subsection{Estimación en Geoestadística}

Usaremos libremente los conceptos y resultados de la Sección 2.5 a 2.10 .

Sean: $E=\mathbb{R} ; \mathcal{E}$ la $\sigma$-álgebra de Borel de $E ; S=\mathbb{R}^{2} ;(\Omega, \mathcal{F}, \mathbb{R})$ un e.p.; $X=\left\{X_{s}: s \in S\right\}$ un proceso con $X_{s}: \Omega \rightarrow E$ v.a. tal que $X_{s} \in \mathcal{L}^{2}(\Omega, \mathcal{F}, P, \mathbb{R})$.

Supongamos que $X$ es intrínsecamente estacionario (Definición 2.5.1) y que $E\left(X_{s}\right)=\mu \forall s$.

Sea $\gamma_{X}: \mathbb{R}^{2} \rightarrow \mathbb{R}$ la función semivariograma de $X$, esto es:

$$
\gamma_{X}(h):=\frac{1}{2} \operatorname{Var}\left(X_{s+h}-X_{s}\right),
$$

cualquiera sea $s \in S$.

Para $N \in \mathbb{N}$, sea $\mathcal{O}_{N} \subset S$ con $1 \leq \#\left(\mathcal{O}_{N}\right)=N$. Consideramos que el proceso observado es

$$
X_{\mathcal{O}_{N}}:=\left\{X_{s} / s \in \mathcal{O}_{N}\right\}
$$

Si $\tilde{s}=\left(s_{1}, s_{2}\right)$ y $\tilde{t}=\left(t_{1}, t_{2}\right)$ están en $S$ pondremos

$$
\|\tilde{s}-\tilde{t}\|:=\left(\left(s_{1}-t_{1}\right)^{2}+\left(s_{2}-t_{2}\right)^{2}\right)^{1 / 2} .
$$

Para cada $2 \pi>\delta \geq 0, \Delta \geq 0, r>0, \alpha \in[0,2 \pi)$ sea

$$
\begin{gathered}
V_{\Delta, \delta}(r, \alpha):=\{u(\cos (\beta), \operatorname{sen}(\beta)) / u \geq 0,|u-r| \leq \Delta, \\
|\beta-\alpha| \leq \delta o|\beta-\alpha| \geq 2 \pi-\delta\} . \\
N_{\mathcal{O}_{N}}(\Delta, \delta, r, \alpha):=\left\{(s, t) \in \mathcal{O}_{N} \times \mathcal{O}_{N} / s-t \in V_{\Delta, \delta}(r, \alpha)\right\} .
\end{gathered}
$$


Definición 4.1.1. Sea $h=r(\cos (\alpha)$, sen $(\alpha))$ con $r>0$ y $\alpha \in[0,2 \pi)$. Llamaremos estimador natural empírico de $\gamma_{X}(h)$ basado en $X_{\mathcal{O}_{N}}$ con tolerancia $\delta \geq 0$ (para el ángulo) y $\Delta \geq 0$ (para el radio) a:

$$
\widehat{\gamma_{\mathcal{O}_{N, \delta, \Delta}}}(h):=\frac{1}{2 \#\left(N_{\mathcal{O}_{N}}(\Delta, \delta, r, \alpha)\right)} \sum_{(s, t) \in N_{\mathcal{O}_{N}}(\Delta, \delta, r, \alpha)}\left(X_{s}-X_{t}\right)^{2} .
$$

Proposición 4.1.2. Sean $I_{N}:\{1, \ldots, N\} \rightarrow \mathcal{O}_{N}$ una biyección; $h=r(\cos (\alpha), \operatorname{sen}(\alpha))$ con $r>0$ y $\alpha \in[0,2 \pi)$.

Para simplificar la notación pongamos:

$$
N(h):=N_{\mathcal{O}_{N}}(\Delta, \delta, r, \alpha) .
$$

Para cada $i=1, \ldots, N$ sean:

$$
\begin{aligned}
n_{h}(1, i): & =\#\left(\left\{j /\left(I_{N}(i), I_{N}(j)\right) \in N(h)\right\}\right) ; \\
n_{h}(2, i): & =\#\left(\left\{j /\left(I_{N}(j), I_{N}(i)\right) \in N(h)\right\}\right) ; \\
n_{h}: & =\#(N(h)) .
\end{aligned}
$$

Sea $A_{h}^{\delta, \Delta}$ la matriz simétrica $N \times N$ dada por:

- para $i<j$

$$
A_{h}^{\delta, \Delta}(i, j)= \begin{cases}0 & \text { si } N(h) \cap\left\{\left(I_{N}(i), I_{N}(j)\right),\left(I_{N}(j), I_{N}(i)\right)\right\}=\emptyset . \\ -\frac{1}{n_{h}} & \text { c.c. }\end{cases}
$$

- $\operatorname{para} i=1, \ldots, N$ :

$$
A_{h}^{\delta, \Delta}(i, i)=\frac{n_{h}(1, i)+n_{h}(2, i)}{n_{h}} .
$$

Entonces:

$$
2 \widehat{\gamma_{\mathcal{O}_{N, \delta, \Delta}}}(h)=\tilde{X}_{\mathcal{O}_{N}}^{\prime} A_{h}^{\delta, \Delta} \tilde{X}_{\mathcal{O}_{N}}
$$

donde

$$
\tilde{X}_{\mathcal{O}_{N}}^{\prime}=\left(X_{I_{N}(1)}, \ldots, X_{I_{N}(N)}\right) .
$$

Demostración. Ejercicio.

Proposición 4.1.3 (Distribución de $\widehat{\gamma_{\mathcal{O}_{N, \delta, \Delta}}}(h)$ para un proceso gaussiano). Continuación de la Proposición 4.1.2. Sean: $\tilde{X}_{\mathcal{O}_{N}} \sim$ $\mathcal{N}(\tilde{0}, \xi)$ con $\Sigma$ definida positiva; $\lambda_{1}, \ldots, \lambda_{k}$ los autovalores no nulos de $A_{h}^{\delta, \Delta} \sum$ (necesariamente $\left.k \leq n_{h}\right)$. Entonces

$$
\widehat{\gamma_{\mathcal{O}_{N, \delta, \Delta}}} \sim \sum_{i=1}^{k} \lambda_{i} \chi_{i, 1}^{2}
$$


donde $\chi_{1,1}^{2}, \ldots, \chi_{k, 1}^{2}$ son v.a.i.i.d. $\chi_{1}^{2}$. Luego:

$$
E\left(\widehat{\mathcal{O}_{N, \delta, \Delta}}(h)\right)=\frac{1}{2} \operatorname{tr}\left(A_{h}^{\delta, \Delta} \Sigma\right),
$$

$y$

$$
\operatorname{Var}\left(\widehat{\gamma_{\mathcal{O}_{N, \delta, \Delta}}}(h)\right)=\frac{1}{2} \operatorname{tr}\left(\left(A_{h}^{\delta, \Delta} \Sigma\right)^{2}\right) .
$$

Demostración. Sea $V$ matriz $N \times N$ tal que

$$
\S=V V^{\prime} .
$$

Sea

$$
Y=V^{-1} \tilde{X}_{\mathcal{O}_{N}}
$$

Entonces:

$$
\operatorname{Cov}(Y)=V^{-1} V V^{\prime}\left(V^{-1}\right)^{\prime}=I_{N}
$$

$\left(I_{N}\right.$ es la identidad $\left.N \times N\right)$.

Luego

$$
Y \sim \mathcal{N}\left(\tilde{0}, I_{N}\right) .
$$

Sea

$$
\Gamma:=V^{\prime} A_{h} V .
$$

Entonces por la Proposición 4.1.2:

$$
\begin{aligned}
2 \widehat{\gamma_{\mathcal{O}}}(h)= & \tilde{X}_{\mathcal{O}_{N}}^{\prime} A_{h} \tilde{X}_{\mathcal{O}_{N}}=\tilde{X}_{\mathcal{O}_{N}}^{\prime}\left(V^{\prime}\right)^{-1} V^{\prime} A_{h} V V^{-1} \tilde{X}_{\mathcal{O}_{N}} \\
& =\tilde{X}_{\mathcal{O}_{N}}^{\prime}\left(V^{\prime}\right)^{-1} \Gamma V^{-1} \tilde{X}_{\mathcal{O}_{N}}=Y^{\prime} \Gamma Y .
\end{aligned}
$$

Sea $P$ matriz $N \times N$ ortogonal tal que

$$
\Gamma=P^{\prime} D P
$$

con $D$ matriz diagonal $N \times N$ de la forma

$$
\left[\begin{array}{cccc}
a_{1} & \cdots & 0 & \\
\vdots & \ddots & \vdots & 0 \\
0 & \cdots & a_{r} & \\
& 0 & & 0
\end{array}\right]
$$

con $r=\operatorname{rango}(\Gamma)$ y $a_{j} \neq 0$ para $j=1, \ldots, r$ son los autovalores (no nulos) de $\Gamma$.

Sea

$$
Z=P Y \text {. }
$$


Por (6) tenemos

$$
2 \widehat{\gamma_{\mathcal{O}}}(h)=Y^{\prime} P^{\prime} D P Y=Z^{\prime} D Z
$$

Como $P$ es ortogonal y por (4) $Y=\left(Y_{1}, \ldots, Y_{N}\right)^{\prime}$ esta formado por v.a.i.i.d. $\mathcal{N}(0,1)$ resulta la misma cosa para $Z=\left(Z_{1}, \ldots, Z_{N}\right)^{\prime}$.

Por (6) se tiene entonces

$$
2 \widehat{\gamma_{\mathcal{O}}}(h)=\sum_{j=1}^{r} a_{j} Z_{j}^{2} .
$$

Luego como $Z_{1}, \ldots, Z_{N}$ son v.a.i.i.d. con distribución $\mathcal{N}(0,1)$ tenemos que $Z_{1}^{2}, \ldots, Z_{r}^{2}$ son v.a.i.i.d. con distribución $\chi_{1}^{2}$. Luego

$$
E\left(2 \widehat{\gamma_{\mathcal{O}}}(h)\right)=\sum_{j=1}^{r} a_{j},
$$

y

$$
\operatorname{Var}\left(2 \widehat{\gamma_{\mathcal{O}}}(h)\right)=2 \sum_{j=1}^{r} a_{j}^{2} .
$$

Equivalentemente:

$$
E\left(\widehat{\gamma_{\mathcal{O}}}(h)\right)=\frac{1}{2} \sum_{j=1}^{r} a_{j},
$$

y

$$
\operatorname{Var}\left(\widehat{\gamma_{\mathcal{O}}}(h)\right)=\frac{1}{2} \sum_{j=1}^{r} a_{j}^{2} .
$$

Es inmediato ver que la Proporsición 4.1.3 quedará probada si probamos la siguiente:

Afirmación 1: $\lambda$ es autovalor de $\Gamma$ si y sólo si $\lambda$ es autovalor de $A_{h} \nwarrow$

Dem. Afirmación 1: $\lambda$ es a.v. de $\Gamma \Leftrightarrow \exists \tilde{v} \in \mathbb{R}^{N}$ con $\tilde{v} \neq \tilde{0}$ tal que $\Gamma \tilde{v}=\lambda \tilde{v} \Leftrightarrow V^{\prime} A_{h} V \tilde{v}=\lambda \tilde{v} \Leftrightarrow$

$$
A_{h} V \tilde{v}=\lambda\left(V^{\prime}\right)^{-1} \tilde{v}
$$

Ahora:

$$
A_{h} V \tilde{v}=A_{h} V V^{\prime}\left(V^{\prime}\right)^{-1} \tilde{v}=A_{h} \Sigma\left(V^{\prime}\right)^{-1} \tilde{v} .
$$

Por (13) tenemos entonces:

$A_{h} \Sigma\left(V^{\prime}\right)^{-1} \tilde{v}=\lambda\left(V^{\prime}\right)^{-1} \tilde{v} \Leftrightarrow \lambda$ es a.v. de $A_{h} \ll$ pues $\left(V^{\prime}\right)^{-1} \tilde{v} \neq \tilde{0}$. 


\subsubsection{El caso isotrópico}

Supongamos que

$$
\gamma_{X}\left(h_{1}\right)=\gamma_{X}\left(h_{2}\right) \quad \text { si } \quad\left\|h_{1}\right\|=\left\|h_{2}\right\| .
$$

Definición 4.1.4. Sea $\gamma_{X}^{\text {iso }}:(0,+\infty) \rightarrow \mathbb{R}$ dada por:

$$
\gamma_{X}^{i s o}(r):=\frac{1}{2} \operatorname{Var}\left(X_{s+h}-X_{s}\right)
$$

con $h \in \mathbb{R}^{2}$ tal que $\|h\|=r$.

Para cada $r>0$ y $\Delta \geq 0$ sea

$$
V_{\Delta}(r):=\left\{h \in \mathbb{R}^{2} /|\|h\|-r| \leq \Delta\right\} .
$$

Recordemos el orden lexicográfico en $\mathbb{R}^{2}$ :

Sean: $\tilde{s}:=\left(s_{1}, s_{2}\right) \in \mathbb{R}^{2}, \quad \tilde{t}=\left(t_{1}, t_{2}\right) \in \mathbb{R}^{2}$.

Ponemos

$$
\tilde{s} \prec \tilde{t} \quad \text { si } \quad s_{2}<t_{2} \text { o } s_{1}<t_{1} \quad \text { si } \quad s_{2}=t_{2} .
$$

Definimos

$$
N_{\mathcal{O}_{N}}(\Delta, r):=\left\{(s, t) \in \mathcal{O}_{N} \times \mathcal{O}_{N} / s \prec t \mathrm{y}(s, t) \in V_{\Delta}(r)\right\} .
$$

Definición 4.1.5. Sea $r>0$ y $\Delta \geq 0$. Llamaremos estimador empírico de $\gamma_{X}^{i s o}(r)$ basado en $X_{\mathcal{O}}$ con tolerancia $\Delta \geq 0$ a:

$$
\widehat{\gamma_{\mathcal{O}_{N, \Delta}^{i s o}}}(r):=\frac{1}{2 \#\left(N_{\mathcal{O}_{N}}(\Delta, r)\right)} \sum_{(s, t) \in N_{\mathcal{O}_{N}}(\Delta, r)}\left(X_{s}-X_{t}\right)^{2} .
$$

Proposición 4.1.6. Análoga a la Proposición 4.1.2 para el caso isotrópico. Sean: $N=\#\left(\mathcal{O}_{N}\right) ; I_{N}:\{1, \ldots, N\} \rightarrow \mathcal{O}_{N}$ una biyección tal que:

$$
i<j \Rightarrow I_{N}(i) \prec I_{N}(j) \text {. }
$$

Sean $r>0$ y $\Delta \geq 0$. Para simplificar la notación pongamos:

$$
N_{r}:=N_{\mathcal{O}_{N}}(\Delta, r) \text {. }
$$

Para cada $i=1, \ldots, N$ sea:

$$
n_{r}(1, i):=\#\left(\left\{j / i<j e\left(I_{N}(i), I_{N}(j)\right) \in N_{r}\right\}\right) .
$$

Para cada $i=2, \ldots, N$ sea:

$$
n_{r}(2, i):=\#\left(\left\{j / j<i \text { e }\left(I_{N}(j), I_{N}(i)\right) \in N_{r}\right\}\right)
$$

Sea

$$
n_{r}:=\#\left(N_{r}\right) .
$$

Sea $A_{r}^{\Delta}$ la matriz simétrica $N \times N$ dada por: 
- para $i<j$

$$
A_{r}^{\Delta}(i, j)= \begin{cases}0 & \text { si }\left(I_{N}(i), I_{N}(j)\right) \notin N_{r} \\ -\frac{1}{n_{r}} & \text { c.c. }\end{cases}
$$

- para $i=1, \ldots, N$ :

$$
A_{r}^{\Delta}(i, i)=\frac{n_{r}(1, i)+n_{r}(2, i)}{n_{r}} .
$$

En este caso $n_{r}(1, N)=n_{r}(2,1)=0$, por definición.

Entonces:

$$
\widehat{2 \gamma_{\mathcal{O}_{N, \Delta}}^{i s o}}(r)=\tilde{X}_{\mathcal{O}_{N}}^{\prime} A_{r}^{\Delta} \tilde{X}_{\mathcal{O}_{N}}
$$

donde

$$
\tilde{X}_{\mathcal{O}_{N}}^{\prime}=\left(X_{I_{N}(1)}, \ldots, X_{I_{N}(N)}\right) .
$$

Proposición 4.1.7. Análoga a la Proposición 4.1.3 para el caso isotrópico. Es la misma que la Proposición 4.1 .3 cambiando $A_{h}^{\delta, \Delta} \sum$ por $A_{r}^{\Delta} \sum y n_{h}$ por $n_{r}$.

\subsection{Estimación paramétrica en el caso isotró- pico fijando $k$ distancias: $0<r_{1}<\ldots<r_{k}$ $(k<\infty)$}

Para cada $\Lambda \in \mathcal{S}:=\{\Lambda \subset S / 1 \leq \#(\Lambda)<\infty\}$ sea $P_{X_{\Lambda}}$ la probabilidad de distribución de $X_{\Lambda}$ sobre $\left(E^{\Lambda}, \mathcal{E}^{\Lambda}\right)$ bajo $P$; es decir:

$$
P_{X}(B)=P\left(X_{\Lambda} \in B\right), \quad B \in \mathcal{E}^{\Lambda} .
$$

Por el Teorema de Kolmogorov sabemos que existe una única probabilidad sobre $\left(E^{S}, \mathcal{E}^{S}\right)$, que denotamos por $P_{X}$ y tal que:

$$
P_{X}\left(\sigma_{\Lambda}^{-1}(B)\right)=P_{X_{\Lambda}}(B), \quad B \in \mathcal{E}^{\Lambda}, \forall \Lambda \in \mathcal{S} .
$$

Sea $\mathcal{P}$ una familia de probabilidades sobre $\left(E^{S}, \mathcal{E}^{S}\right)$ de la que sabemos que $P_{X} \in \mathcal{P}$.

Supongamos que $\exists \Theta \subset \mathbb{R}^{p}$ con $p \geq 1$ abierto tal que existe una biyección entre $\mathcal{P}$ y $\Theta$. Pondremos entonces

$$
\mathcal{P}=\left\{\mu_{\theta} / \theta \in \Theta\right\} .
$$

Consideramos ahora el caso isotrópico, esto es

$$
\gamma_{X}\left(h_{1}\right)=\gamma_{X}\left(h_{2}\right) \quad \text { si } \quad\left\|h_{1}\right\|=\left\|h_{2}\right\| .
$$

Pondremos entonces

$$
\gamma_{X}^{i s o}(r)=\gamma_{X}(h) \quad \text { con } h \text { tal que }\|h\|=r, \text { con } r \geq 0 .
$$


Para cada $r \geq 0$, y cada $\theta \in \Theta$ sea

$$
\gamma(r, \theta)=\gamma_{X}^{i s o}(r) \quad \text { si } P_{X}=\mu_{\theta}
$$

Supongamos fijados $k \geq 1$ entero y $0<r_{1}<\ldots<r_{k}$. Queremos estudiar la estimación de $\gamma\left(r_{1}, \theta\right), \ldots, \gamma\left(r_{k}, \theta\right)$ cuando $P_{X}=\mu_{\theta}$.

Pondremos: $\tilde{\gamma}(\theta):=\left(\gamma\left(r_{1}, \theta\right), \ldots, \gamma\left(r_{k}, \theta\right)\right)^{\prime}, \theta \in \Theta$.

Veremos también este problema de inferencia suponiendo que se cumple alguno de los modelos para la función semivariograma definidos en el Capítulo 2.

Continuaremos usando conceptos, resultados y anotaciones de la Sección 4.1 .

Pongamos:

con $\Delta \geq 0$ fijo.

$$
\widehat{\tilde{\gamma}_{N, \Delta}^{\text {iso }}}:=\left(\widehat{\tilde{\gamma}_{N, \Delta}^{\text {iso }}}\left(r_{1}\right), \ldots, \widehat{\tilde{\gamma}_{N, \Delta}^{\text {iso }}}\left(r_{k}\right)\right)^{\prime}
$$

Definición 4.2.1. Llamaremos estimador de mínimos cuadrados de $\tilde{\gamma}(\theta)$ basado en $\widehat{\tilde{\gamma}_{N, \Delta}^{\text {iso }}}$ al vector aleatorio

$$
\tilde{\gamma}\left(\hat{\theta}_{M C, \Delta, N}\right):=\left(\gamma\left(r_{1}, \hat{\theta}_{M C, \Delta, N}\right), \cdots, \gamma\left(r_{k}, \hat{\theta}_{M C, \Delta, N}\right)\right)^{\prime}
$$

tal que:

$$
\left.\sum_{i=1}^{k}\left(\widehat{\gamma_{\mathcal{O}_{N, \Delta}}^{\text {iso }}}\left(r_{i}\right)-\gamma\left(r_{i}, \hat{\theta}_{M C, \Delta, N}\right)\right)^{2} \leq \sum_{i=1}^{k} \widehat{\left(\gamma_{\mathcal{O}_{N, \Delta}}^{\text {sso }}\right.}\left(r_{i}\right)-\gamma\left(r_{i}, \theta\right)\right)^{2} \quad \forall \theta \in \Theta .
$$

Ejemplo 4.2.2 (Modelo a) de la Sección 2.7). Sean : $\Theta=(0,+\infty)$;

$$
\gamma(r, \theta)=\theta \quad \forall r>0 .
$$

En este caso, se puede ver fácilmente que:

$$
\hat{\theta}_{M C, \Delta, N}=\frac{1}{k} \sum_{i=1}^{k} \widehat{\gamma_{\mathcal{O}_{N, \Delta}}^{i s o}}\left(r_{i}\right) .
$$

Ejemplo 4.2.3 (Modelo b) de la Sección 2.7). Sean : $\Theta=(0,+\infty) \times$ $(0,+\infty) ; \gamma:(0,+\infty) \times \Theta \rightarrow(0,+\infty)$ dada por

$$
\gamma\left(r,\left(\theta_{1}, \theta_{2}\right)=\theta_{2}\left(1-\exp \left(-\theta_{1}^{-1} r\right)\right) .\right.
$$

En este caso el estimador de minimos cuadrados $\tilde{\gamma}\left(\left(\hat{\theta}_{1, M C, \Delta}, \hat{\theta}_{2, M C, \Delta}\right)\right)$ de $\tilde{\gamma}\left(\left(\theta_{1}^{0}, \theta_{2}^{0}\right)\right)$ está definido por

$$
\begin{gathered}
\left.\sum_{i=1}^{k} \widehat{\left(\gamma_{\mathcal{O}_{N, \Delta}}^{\text {iso }}\right.}\left(r_{i}\right)-\widehat{\theta}_{2, M C, \Delta}\left(1-\exp \left(-\widehat{\theta}_{1, M C, \Delta}^{-1} r_{i}\right)\right)\right)^{2} \\
\quad \leq \sum_{i=1}^{k}\left(\widehat{\gamma_{\mathcal{O}_{N, \Delta}}^{\text {iso }}}\left(r_{i}\right)-\theta_{2}\left(1-\exp \left(-\theta_{1}^{-1} r_{i}\right)\right)\right)^{2}
\end{gathered}
$$

$\forall\left(\theta_{1}, \theta_{2}\right) \in \Theta$ 


\subsubsection{Estimador de mínimos cuadrados generalizado}

Para cada $\theta \in \Theta$, sea

$$
\Sigma_{\theta}\left(\widehat{\tilde{\gamma}_{N, \Delta}^{i s o}}\right)
$$

la matriz de covarianza de $\widehat{\tilde{\gamma}_{N, \Delta}^{\text {iso }}}$ suponiendo $P_{X}=\mu_{\theta}$.

Definición 4.2.4. Llamaremos estimador de mínimos cuadrados generalizado de $\tilde{\gamma}(\theta)$ basado en $\widehat{\tilde{\gamma}_{N, \Delta}^{\text {iso }}}$ al vector aleatorio $\tilde{\gamma}\left(\hat{\theta}_{G L S, \Delta, N}\right)$ tal que:

$$
\begin{gathered}
\left(\widehat{\tilde{\gamma}_{N, \Delta}^{i s o}}-\tilde{\gamma}\left(\hat{\theta}_{G L S, \Delta, N}\right)\right)^{\prime}\left[\Sigma_{\theta_{G L S, \Delta, N}}\left(\widehat{\tilde{\gamma}_{N, \Delta}^{i s o}}\right)\right]\left(\widehat{\tilde{\gamma}_{N, \Delta}^{i s o}}-\tilde{\gamma}\left(\hat{\theta}_{G L S, \Delta, N}\right)\right) \\
\leq\left(\widehat{\tilde{\gamma}_{N, \Delta}^{i s o}}-\tilde{\gamma}(\theta)\right)^{\prime}\left[\Sigma_{\theta}\left(\widehat{\tilde{\gamma}_{N, \Delta}^{i s o}}\right)\right]\left(\widehat{\tilde{\gamma}_{N, \Delta}^{i s o}}-\tilde{\gamma}(\theta)\right) \quad \forall \theta \in \Theta .
\end{gathered}
$$

La mayoría de las veces es difícil obtener $\Sigma_{\theta}\left(\widehat{\tilde{\gamma}_{N, \Delta}^{\text {so }}}\right)$, por ello se suele usar un estimador de mínimos cuadrados ponderados con ponderaciones basadas en $\operatorname{Var}_{\theta}\left(\widehat{\gamma_{\mathcal{O}_{N, \Delta}}^{\text {iso }}}\left(r_{i}\right)\right)$, para $i=1, \ldots, k$ y $\theta \in \Theta$.

No obstante, veamos una definición más general.

Definición 4.2.5. Para cada $i=1, \ldots, k$ sea $w_{i}:(0,+\infty) \rightarrow(0,1)$ tales que:

$$
\sum_{i=1}^{k} w_{i}(t)=1 \quad \forall t \in(0,+\infty) .
$$

Llamaremos estimador de mínimos cuadrados de $\tilde{\gamma}(\theta)$ ponderado por el sistema de pesos $w_{1}, \ldots, w_{k}$ y basado en $\widehat{\tilde{\gamma}_{N, \Delta}^{i s o}}$ a $\tilde{\gamma}\left(\hat{\theta}_{W L S, N, \Delta}\right)$ tal que:

$$
\begin{gathered}
\left.\left.\sum_{i=1}^{k} w_{i}\left(\operatorname{Var}_{\hat{\theta}_{W L S, N, \Delta}} \widehat{\left(\widehat{\gamma_{\mathcal{O}_{N, \Delta}}^{\text {iso }}}\right.}\left(r_{i}\right)\right)\right) \widehat{\left(\gamma_{\mathcal{O}_{N, \Delta}}^{\text {iso }}\right.}\left(r_{i}\right)-\gamma\left(r_{i}, \hat{\theta}_{W L S, N, \Delta}\right)\right)^{2} \leq \\
\sum_{i=1}^{k} w_{i}\left(\operatorname{Var}_{\theta}\left(\widehat{\gamma_{\mathcal{O}_{N, \Delta}}^{\text {iso }}}\left(r_{i}\right)\right)\right)\left(\widehat{\gamma_{\mathcal{O}_{N, \Delta}}^{\text {iso }}}\left(r_{i}\right)-\gamma\left(r_{i}, \theta\right)\right)^{2} \quad \forall \theta \in \Theta .
\end{gathered}
$$

\subsubsection{Estimador de mínimo contraste}

Veremos ahora una clase de estimadores de la función semivariograma en $r_{1}, \ldots, r_{k}$ de la que los estimadores vistos hasta el momento pueden considerarse como casos particulares.

Sea $\mathcal{M}_{k}:=\{V / V$ es una matriz $\mathrm{k} \times \mathrm{k}$ simétrica definida positiva $\}$.

Sea $V: \Theta \rightarrow \mathcal{M}_{k}$. Sea $U_{V}: \Theta \times E^{\mathcal{O}_{N}} \rightarrow \mathbb{R}$ dado por:

$$
\left.U_{V}\left(\theta, X_{\mathcal{O}_{N}}\right)=\left(\widehat{\tilde{\gamma}_{\mathcal{O}_{N}, \Delta}^{\text {iso }}}-\tilde{\gamma}(\theta)\right)^{\prime} V(\theta) \widehat{\left(\tilde{\gamma}_{\mathcal{O}_{N}, \Delta}^{\text {iso }}\right.}-\tilde{\gamma}(\theta)\right) .
$$


Definición 4.2.6. Llamaremos estimador de $\tilde{\gamma}(\theta)$ de mínimo contraste dado por $V$ basado en $\widehat{\tilde{\gamma}_{N, \Delta}^{i s o}}$ al vector aleatorio $\tilde{\gamma}\left(\hat{\theta}_{U_{V}, \Delta, N}\right)$ tal que:

$$
U_{V}\left(\hat{\theta}_{U_{V}, \Delta, N}, X_{\mathcal{O}_{N}}\right) \leq U_{V}\left(\theta, X_{\mathcal{O}_{N}}\right) \quad \forall \theta \in \Theta .
$$

En cada caso particular, $V(\theta)$ podría ser un parámetro de $\widehat{\tilde{\gamma}_{\mathcal{O}_{N}, \Delta}^{\text {iso }}}$ cuando se supone $P_{X}=\mu_{\theta}$.

Por ejemplo, en el caso del estimador de mínimos cuadrados generalizado:

$$
V(\theta)=\left[\aleph_{\theta}\left(\widehat{\tilde{\gamma}_{\mathcal{O}_{N}, \Delta}^{\text {iso }}}\right]^{-1}\right.
$$

\subsection{Estimación de la función semivariograma bajo presencia de covariable}

Supongamos que existe otro proceso $Z=\left\{Z_{s} / s \in S\right\}$ definido sobre $(\Omega, \mathcal{F}, P)$ con valores en $\left(H^{S}, \mathcal{H}^{S}\right)$ siendo:

$$
H=\mathbb{R}^{p}, p \geq 1 \quad \text { y } \quad \mathcal{H}:=\mathcal{B}_{p} .
$$

Supongamos que $\exists \delta \in \mathbb{R}^{p}$ tal que

$$
X_{s}=Z_{s}^{t} \delta+\varepsilon_{s}
$$

con $\varepsilon=\left\{\varepsilon_{s} / s \in S\right\}$ un proceso intrínsecamente estacionario de $2^{o}$ orden $\operatorname{con} E\left(\varepsilon_{s}\right)=0 \forall s$ y

$$
E\left(\left(\varepsilon_{s+h}-\varepsilon_{s}\right)^{2}\right)=2 \gamma(h, \theta) \quad \forall s, \forall h \text { cuando } P_{X}=\mu_{\theta} .
$$

Suponiendo que $Z$ es un proceso observado.

Generalmente $\delta$ es un parámetro que debe ser estimado ya sea independientemente de $\tilde{\gamma}(\theta)$ o bien simultáneamente.

Un método podría ser el siguiente:

1. Estimar $\delta$ por medio de $\widehat{\delta}_{M C}$ de mínimos cuadrados. Esto es con $\widehat{\delta}_{M C}$ tal que

$$
\sum_{t \in \mathcal{O}_{N}}\left(X_{t}-Z_{t}^{\prime} \widehat{\delta}_{M C}\right)^{2} \leq \sum_{t \in \mathcal{O}_{N}}\left(X_{t}-Z_{t}^{\prime} \delta\right)^{2} \quad \forall \delta .
$$

2. Definir $\widehat{\varepsilon}_{t}:=X_{t}-Z_{t}^{\prime} \widehat{\delta}_{M C} \forall t$.

3. Aplicar los estimadores de semivariograma vistos en la Sección 4.2 al proceso $\left\{\widehat{\varepsilon}_{t} / t \in S\right\}$. 


\subsection{Estimación de Máxima Verosimilitud}

Supongamos que para cada $\theta \in \Theta, \mathbb{Z}(\theta)$ es una matriz simétrica $N \times N$ definida positiva.

Si suponemos $\tilde{X}_{\mathcal{O}_{N}} \sim \mathcal{N}(\mu, \Sigma)$, entonces suponemos que $\Sigma \in$ $\{\mathbb{Z}(\theta) / \theta \in \Theta\}$; luego $\theta$ es un parámetro a estimar. El estimador de máxima verosimilitud basado en $\tilde{X}_{\mathcal{O}_{N}}$ de $\theta$ es $\widehat{\theta}_{M V}$ tal que

$$
l_{\mathcal{O}_{N}}\left(\widehat{\theta}_{M V}\right) \geq l_{\mathcal{O}_{N}}(\theta) \quad \theta \in \Theta,
$$

siendo

$$
l_{\mathcal{O}_{N}}(\theta):=-\frac{1}{2}\left\{\ln (\operatorname{det}(\Sigma(\theta)))+\left(\tilde{X}_{\mathcal{O}_{N}}-\mu\right)^{\prime} \Sigma(\theta)^{-1}\left(\tilde{X}_{\mathcal{O}_{N}}-\mu\right)\right\} .
$$

En tal caso, llamaremos estimador de máxima verosimilitud de $\tilde{\gamma}(\theta)$ basado en $\tilde{X}_{\mathcal{O}_{N}}$ a $\tilde{\gamma}\left(\hat{\theta}_{M V}\right)$.

\subsection{Validación de modelos para función de semivariograma en el caso isotrópico}

Como vimos en la Sección 4.2:

$$
\gamma(r, \theta)=\gamma_{X}^{i s o}(r) \quad \text { si } P_{X}=\mu_{\theta} .
$$

Supongamos que $X$ es $w-\mathcal{L}^{2}$ y que $\mu_{X}=0$ ( esto es equivalente a suponer $\mu_{X}$ conocido y $X$ centrado).

Por la Proposición 2.5.2 tenemos (a partir de (4.2)):

$$
\gamma(r, \theta)=\sigma_{X}^{2}-\operatorname{Cov}\left(X_{s+h}, X_{s}\right)
$$

con $s \in \mathbb{Z}^{2},\|h\|=r \mathrm{y}$

$$
\sigma_{X}^{2}=\operatorname{Var}\left(X_{s}\right)
$$

cualquiera sea $s \in \mathbb{Z}^{2}$.

Supongamos ahora

$H_{0}$ : Conocemos $\sigma_{X}^{2}, \gamma(\cdot, \theta)$ y $\theta \in \Theta$ tal que $P_{X}=\mu_{\theta}$.

A partir de este supuesto y de las observaciones $\tilde{X}_{\mathcal{O}_{N}}$.

Definiremos dos métodos de validación de $H_{0}$.

\subsubsection{Validación cruzada de $H_{0}$}

Pongamos

$$
\mathcal{O}_{N}:=\left\{s_{1}, \ldots, s_{N}\right\} .
$$

Para cada $i=1, \ldots, N$ sea

$$
\mathcal{O}_{N, i}:=\mathcal{O}_{N} \backslash\left\{s_{i}\right\} .
$$


Sea $\hat{X}_{s_{i}}$ el kriging simple para estimar $X_{s_{i}}$ basándose en $\tilde{X}_{\mathcal{O}_{N, i}}=$ $\left(X_{s_{1}}, \ldots, X_{s_{i-1}}, X_{s_{i+1}}, \ldots, X_{s_{N}}\right)^{\prime}$.

Por lo visto en la Sección 2.7 tenemos:

$$
\hat{X}_{s_{i}}=\tilde{c}_{i}^{\prime}\left(\Sigma_{i}\right)^{-1} \tilde{X}_{\mathcal{O}_{N, i}}
$$

donde

$$
\begin{aligned}
\tilde{c}_{i}:= & \left(\operatorname{Cov}\left(X_{s_{1}}, X_{s_{i}}\right), \ldots, \operatorname{Cov}\left(X_{s_{i-1}}, X_{s_{i}}\right),\right. \\
& \left.\operatorname{Cov}\left(X_{s_{i+1}}, X_{s_{i}}\right), \ldots, \operatorname{Cov}\left(X_{s_{N}}, X_{s_{i}}\right)\right)^{\prime},
\end{aligned}
$$

y

$$
\aleph_{i}=\nwarrow\left(\mathcal{O} \tilde{X}_{\mathcal{O}_{N, i}}\right)
$$

Por la Proposición 2.17.2 se tiene que:

$$
\tilde{\sigma}_{s_{i}}^{2}:=E\left(\left(\hat{X}_{s_{i}}-X_{s_{i}}\right)^{2}\right)=\sigma_{X}^{2}-\tilde{c}^{\prime} \Sigma^{-1} \tilde{c} .
$$

Para verificar la hipótesis $H_{0}$ usamos el estadístico

$$
T:=\frac{1}{N} \sum_{i=1}^{N} \frac{\left(\hat{X}_{s_{i}}-X_{s_{i}}\right)^{2}}{\tilde{\sigma}_{s_{i}}^{2}} .
$$

Luego, si la hipótesis $H_{0}$ es verdadera debe cumplirse $T \simeq 1$.

Suponiendo que $X$ es gaussiano, y que

$$
X_{s_{i}}=\tilde{c}_{i}^{\prime}\left(\Sigma_{i}\right)^{-1} \tilde{X}_{\mathcal{O}_{N, i}}+\varepsilon_{i}
$$

$\operatorname{con} \varepsilon_{i}$ gaussiana y que

$$
\hat{X}_{s_{1}}-X_{s_{1}}, \ldots, \hat{X}_{s_{N}}-X_{s_{N}}
$$

son independientes, tenemos

$$
\frac{\left(\hat{X}_{s_{1}}-X_{s_{1}}\right)^{2}}{\tilde{\sigma}_{s_{1}}^{2}}, \ldots, \frac{\left(\hat{X}_{s_{N}}-X_{s_{N}}\right)^{2}}{\tilde{\sigma}_{s_{N}}^{2}}
$$

son v.a.i.i.d. $\chi_{1}^{2}$.

Luego:

$$
N T=\sum_{i=1}^{N} \frac{\left(\hat{X}_{s_{i}}-X_{s_{i}}\right)^{2}}{\tilde{\sigma}_{s_{i}}^{2}} \sim \chi_{N}^{2} .
$$

Sea

$$
q(N, \beta)=F_{\chi_{N}^{2}}^{-1}(\beta) \quad \forall \beta \in(0,1),
$$

donde $F_{\chi_{N}^{2}}$ es la f.d.a de una v.a. con distribución $\chi_{N}^{2}$. 
Entonces, bajo (4.5) el test de nivel $1-\alpha(0<\alpha<1)$ para $H_{0}$ es el que rechaza $H_{0}$ si

$$
N T \leq q\left(N, \frac{\alpha}{2}\right) \quad o \quad N T \geq q\left(N, \frac{1-\alpha}{2}\right) .
$$

Si $X$ no es gaussiano, mejor dicho, si (4.4) no es válida, esta conclusión sobre $N T$ no es válida. Luego este método de validación cruzada es de limitada utilidad.

Veamos el siguiente método aplicable en situaciones más generales.

\subsubsection{Validación de $H_{0}$ por "bootstrap" paramétrico}

Sea $m \in \mathbb{N}$ "grande" (mayor que 30 ).

Para cada $j=1, \ldots, m$ sea

$$
\tilde{Y}_{\mathcal{O}_{N}}^{(j)}:=\left(Y_{s_{1}}^{(j)}, \ldots, Y_{s_{N}}^{(j)}\right)^{\prime}
$$

un vector con distribución $\mathcal{N}(\tilde{0}, \S)$.

Bajo $H_{0}$ suponemos $\ \Sigma$ definida por (4.3).

Para cada $j=1, \ldots, N$ sea

$$
\gamma_{\mathcal{O}_{N}, \Delta}^{(j)}(r):=\frac{\sum_{(s, t) \in N_{\mathcal{O}_{N}}(\Delta, r)}\left(Y_{s}^{(j)}-Y_{t}^{(j)}\right)^{2}}{2 \#\left(N_{\mathcal{O}_{N}}(\Delta, r)\right)},
$$

para $r=r_{1}, \ldots, r_{k}$.

Definimos:

$$
\gamma_{\mathcal{O}_{N}, \Delta}^{\min }(r):=\min \left(\left\{\gamma_{\mathcal{O}_{N}, \Delta}^{(j)}(r) / j: 1, \ldots, m\right\}\right),
$$

y

$$
\gamma_{\mathcal{O}_{N}, \Delta}^{\max }(r):=\max \left(\left\{\gamma_{\mathcal{O}_{N}, \Delta}^{(j)}(r) / j: 1, \ldots, m\right\}\right),
$$

para $r=r_{1}, \ldots, r_{k}$.

Supongamos ahora que $\gamma_{\mathcal{O}_{N}}^{(j)}, j=1, \ldots, m$ son independientes.

Entonces, para cada $r: \gamma_{\mathcal{O}_{N}, \Delta}^{(j)}(r)$ con $j=1, \ldots, m$ son v.a. independientes.

El estadístico que usamos se basa en el siguiente resultado cuya demostración queda como ejercicio.

Lema 4.5.1. Sean $Z_{1}, \ldots, Z_{m+1}$ v.a.i.i.d.. Entonces

$$
P\left(Z_{1}=\min \left(Z_{1}, \ldots, Z_{m+1}\right)\right) \leq \frac{1}{m+1} .
$$


Se puede ver que este resultado es equivalente a:

Sean $Z, Z_{1}, \ldots, Z_{m}$ v.a.i.i.d. Entonces:

$$
\begin{gathered}
P\left(Z<\min \left(Z_{1}, \ldots, Z_{m}\right)\right) \leq \frac{1}{m+1} \\
\text { y } \\
P\left(Z>\max \left(Z_{1}, \ldots, Z_{m}\right)\right) \leq \frac{1}{m+1} .
\end{gathered}
$$

Definición de test:

1. Generar muestras $\tilde{y}_{\mathcal{O}_{N}}^{(j)} j=1, \ldots, m$ de $\tilde{Y}_{\mathcal{O}_{N}}^{(j)}$ satisfaciendo (4.6).

2. Para cada $j=1, \ldots, m$ y $r=r_{1}, \ldots, r_{k}$ definir:

$$
\widehat{\gamma_{\mathcal{O}_{N}, \Delta}^{(j)}}(r):=\frac{1}{2 \#\left(N_{\mathcal{O}_{N}}(\Delta, r)\right)} \sum_{(s, t) \in N_{\mathcal{O}_{N}}(\Delta, r)}\left(y_{s}^{(j)}-y_{t}^{(j)}\right)^{2} .
$$

3. Definir para cada $r=r_{1}, \ldots, r_{k}$ :

$$
\widehat{\gamma_{\mathcal{O}_{N}, \Delta}^{\min }}(r):=\min \left(\left\{\widehat{\gamma_{\mathcal{O}_{N}, \Delta}^{(j)}}(r) / j: 1, \ldots, m\right\}\right),
$$

$\mathrm{y}$

$$
\left.\widehat{\gamma_{\mathcal{O}_{N}, \Delta}^{\max }}(r):=\max \left(\widehat{\left\{\gamma_{\mathcal{O}_{N}, \Delta}^{(j)}\right.}(r) / j: 1, \ldots, m\right\}\right) .
$$

Sea $\widehat{\gamma_{\mathcal{O}_{N}, \Delta}^{\text {iso }}}(r)$ para cada $r=r_{1}, \ldots, r_{k}$ como fue definido en la Definición 4.1.5.

4. Rechazar $H_{0}$ si para algún $r=r_{1}, \ldots, r_{k}$ se tiene

$$
\widehat{\gamma_{\mathcal{O}_{N}, \Delta}^{i s o}}(r)<\widehat{\gamma_{\mathcal{O}_{N}, \Delta}^{\min }}(r) \quad o \quad \widehat{\gamma_{\mathcal{O}_{N}, \Delta}^{i s o}}(r)>\widehat{\gamma_{\mathcal{O}_{N}, \Delta}^{\max }}(r) .
$$

Notemos que la única razón para suponer $\tilde{Y}_{\mathcal{O}_{N}}^{(j)}$ gaussiano es para facilitar el paso 1 de la definición de test. Lo importante es que $\Sigma\left(\tilde{Y}_{\mathcal{O}_{N}}^{(j)}\right)=\Sigma$ $\forall j=1, \ldots, m$.

Por el Lema recién mencionado el test aquí propuesto tiene nivel de confianza $\geq 1-\frac{2}{m+1}$.

En ([5]) se dice que este test es relativamente conservador en el sentido de que en la práctica rechaza menos veces de las que debiera.

\subsection{Autocorrelación en redes espaciales}

Sea $S \subset \mathbb{R}^{2}$ numerable.

Sea $\mathcal{V}:=\left\{V_{s} / s \in S\right\}$ un sistema de vecindades de $S$ 
Definición 4.6.1. Sea $\emptyset \neq \mathcal{R} \subset\left\{(s, t) \in S \times S / t \in V_{s}\right\}$.

Sea $W_{\mathcal{R}}: S \times S \rightarrow[0, M]$ con $0<M<\infty$

$$
W_{\mathcal{R}}(s, t):=\left\{\begin{array}{l}
0 \quad \text { si } t=s, s \in S \\
0 \quad \text { si }(s, t) \notin \mathcal{R} \\
0>\quad \text { si }(s, t) \in \mathcal{R} .
\end{array}\right.
$$

Diremos que $W_{\mathcal{R}}$ es una matriz de contigüidad asociada a $R$.

Definición 4.6.2. Sean $\mathcal{R} y W_{\mathcal{R}}$ como en la Definición 4.6.1. Llamaremos matriz de contigüidad normalizada asociada a $R$ a:

$$
W_{\mathcal{R}}^{*}(s, t):=\frac{W_{\mathcal{R}}(s, t)}{\sum_{t^{\prime} \in \mathcal{R}_{s}} W_{\mathcal{R}}\left(s, t^{\prime}\right)},
$$

donde $\mathcal{R}_{s}:=\left\{t^{\prime} \in S /\left(s, t^{\prime}\right) \in \mathcal{R}\right\}$.

Sea $X=\left\{X_{s} / s \in S\right\}$ con $X_{s} \in \mathcal{L}^{2}(\Omega, \mathcal{F}, P, \mathbb{R}), \forall s \in S$.

\subsection{1 Índice de Moran}

Sea $D_{n} \in \mathcal{S}(S):=\{\Lambda \subset S / 1 \leq \#(\Lambda)<\infty\}$ con \# $\left(D_{n}\right)=n \geq 2$ entero.

Pongamos:

$$
\begin{aligned}
S_{0, D_{n}}\left(W_{\mathcal{R}}\right): & =\sum_{s \in D_{n}} \sum_{t \in D_{n}} W_{\mathcal{R}}(s, t) . \\
S_{1, D_{n}}\left(W_{\mathcal{R}}\right): & =\sum_{s \in D_{n}} \sum_{t \in D_{n}}\left(\left(W_{\mathcal{R}}(s, t)\right)^{2}+W_{\mathcal{R}}(s, t) W_{\mathcal{R}}(t, s)\right) .
\end{aligned}
$$

Fijamos $n \in \mathbb{N}$.

Sea $X=\left(X_{s}\right)_{s \in D_{n}}$. Pongamos $D_{n}:=\left\{s_{1}, \ldots, s_{n}\right\}$.

Consideremos que $X_{s} \in \mathcal{L}^{2}(\Omega, \mathcal{F}, \mu, \mathbb{R})$.

$H_{0}: X_{s_{1}}, \ldots, X_{s_{n}}$ son $\mu$-independientes.

Definición 4.6.3. Si $\mu\left(X_{s}\right):=E_{\mu}\left(X_{s}\right)=0 \forall s \in D_{n}$ definimos

$$
C_{W_{\mathcal{R}}, D_{n}, 0}(X):=\sum_{s \in D_{n}} \sum_{t \in D_{n}} W_{\mathcal{R}}(s, t) X_{s} X_{t}
$$

Proposición 4.6.4. Supongamos que $\mu\left(X_{s}\right)=0 \forall s \in D_{n}$ y que se cumple $H_{0}$. Entonces:

$$
\begin{gathered}
\operatorname{Var}_{\mu}\left(C_{W_{\mathcal{R}}, D_{n}, 0}(X)\right) \\
=\sum_{s \in D_{n}} \sum_{t \in D_{n}}\left(\left(W_{\mathcal{R}}(s, t)\right)^{2}+W_{\mathcal{R}}(s, t) W_{\mathcal{R}}(t, s)\right) \mu\left(X_{s}^{2}\right) \mu\left(X_{t}^{2}\right) .
\end{gathered}
$$

Notemos que como $\mu\left(X_{s}\right)=0, \mu\left(X_{s}^{2}\right)=\operatorname{Var}_{\mu}\left(X_{s}\right) \forall s \in D_{n}$. 
Demostración. Ejercicio.

Definición 4.6.5. Supongamos que $\mu\left(X_{s}\right)=0 \forall s \in D_{n}$ y que se conoce $\mu\left(X_{s}^{2}\right) \forall s \in D_{n}$.

Se llama indice de Morán de $X$ (sobre $D_{n}$ ) asociado a $W_{\mathcal{R}}\left(:=\left[W_{\mathcal{R}}(s, t)\right]_{s \in D_{n}, t \in D_{n}}\right)$ y $\mu$ a:

$$
I_{W_{\mathcal{R}}, D_{n}, 0, \mu}^{M}(X):=\frac{C_{W_{\mathcal{R}}, D_{n}, 0}(X)}{\left(\operatorname{Var}_{\mu}\left(C_{W_{\mathcal{R}}, D_{n}, 0}(X)\right)\right)^{1 / 2}} .
$$

Nota 4.6.6. Supongamos que:

i) $\mu\left(X_{s}\right)=0 \forall s \in D_{n}$.

ii) $\mu\left(X_{s}^{2}\right)=a_{s} \sigma^{2}$ con $a_{s}>0$ y $\sigma>0, \forall s \in D_{n}$.

iii) Se cumple $H_{0}$.

Entonces:

$$
\begin{gathered}
\operatorname{Var}_{\mu}\left(C_{W_{\mathcal{R}}, D_{n}, 0}(X)\right) \\
=\sigma^{4} \sum_{s \in D_{n}} \sum_{t \in D_{n}}\left(\left(W_{\mathcal{R}}(s, t)\right)^{2}+W_{\mathcal{R}}(s, t) W_{\mathcal{R}}(t, s)\right) a_{s} a_{t} .
\end{gathered}
$$

Definición 4.6.7. Supongamos que:

i) $\mu\left(X_{s}\right)=0 \forall s \in D_{n}$.

ii) $\mu\left(X_{s}^{2}\right)=a_{s} \sigma^{2}$ con $a_{s}>0$ y $\sigma>0, \forall s \in D_{n}$.

Se llama índice de Moran de $X$ sobre $D_{n}$ asociado a $W_{\mathcal{R}}$, con $\left(a_{s}\right)_{s \in D_{n}}$ conocidos y $\sigma$ desconocido a estimar a:

$$
\begin{aligned}
& I_{W_{\mathcal{R}}, D_{n}, 0,\left(a_{s}\right)_{s \in D_{n}}}^{M}(X):= \\
& :=\frac{C_{W_{\mathcal{R}}, D_{n}, 0}(X)}{\frac{1}{n} \sum_{s \in D_{n}} X_{s}^{2}\left(\sum_{s \in D_{n}} \sum_{t \in D_{n}}\left(\left(W_{\mathcal{R}}(s, t)\right)^{2}+W_{\mathcal{R}}(s, t) W_{\mathcal{R}}(t, s)\right) a_{s} a_{t}\right)^{1 / 2}},
\end{aligned}
$$

Definición 4.6.8. Supongamos que:

i) $\mu\left(X_{s}\right)=\mu_{X} \forall s \in D_{n}$.

ii) $\mu\left(\left(X_{s}-\mu_{X}\right)^{2}\right):=\sigma_{X}^{2}, \forall s \in D_{n}$. 
Si ambos, $\mu_{X}$ y $\sigma_{X}^{2}$ son desconocidos a estimar, se llama índice de Moran de $X$ sobre $D_{n}$ asociado a $W_{\mathcal{R}}$ a:

$$
I_{W_{\mathcal{R}}, D_{n}}^{M}(X):=\frac{n \sum_{s \in D_{n}} \sum_{t \in D_{n}} W_{\mathcal{R}}(0, t)\left(X_{s}-\bar{X}\right)\left(X_{t}-\bar{X}\right)}{S_{0, D_{n}}\left(W_{\mathcal{R}}\right) \sum_{s \in D_{n}}\left(X_{s}-\bar{X}\right)^{2}},
$$

donde:

$$
\overline{X:}=\frac{1}{n} \sum_{s \in D_{n}} X_{s} .
$$

Definición 4.6.9 (d-índice de Moran). Sea $S \subset \mathbb{R}^{2}$ numerable, $\mathcal{V}:=\left\{V_{s} / s \in S\right\}$ un sistema de vecindades de $S ; \mathcal{R}(\mathcal{V}):=\{(s, t) \in$ $\left.S \times S / t \in V_{s}\right\}$.

Sea $W_{\mathcal{R}(\mathcal{V})}: S \times S \rightarrow\{0,1\}$ dada por:

$$
W_{\mathcal{R}(\mathcal{V})}(s, t):= \begin{cases}1 & \text { si }(s, t) \in \mathcal{R}(\mathcal{V}) \\ 0 & \text { c.c. }\end{cases}
$$

a) Sea $d \geq 2$ entero. Se dice que $\left(s_{1}, \ldots, s_{d}\right) \in S^{d}$ es una $\mathcal{V}$-trayectoria entre $s_{1}$ y $s_{d}$ si

$$
W_{\mathcal{R}(\mathcal{V})}\left(s_{i}, s_{i+1}\right)=1 \text { para } i=1, \ldots, d-1 .
$$

b) Seant y $s$ en $S$. Se dice que s y t están $\mathcal{V}$-conectados si existe $\left(s_{1}, \ldots, s_{d}\right) \in$ $S^{d} \mathcal{V}$-trayectoria tal que $s_{1}=s$ y $s_{d}=t$.

c) Sean $t$ y $s$ en $S$. Si s y $t$ están $\mathcal{V}$-conectados, definimos la $\mathcal{V}$-distancia entre $t$ y $s$ a

$$
d_{\mathcal{V}}(s, t):=\min D_{\mathcal{V}}(s, t)-1
$$

siendo

$$
\begin{aligned}
D_{\mathcal{V}}(s, t):=\{ & d \geq 2 / \exists\left(s_{1}, \ldots, s_{d}\right) \in S^{d} \\
& \left.\mathcal{V} \text {-trayectoria con } s_{1}=s \text { y } s_{d}=t\right\} .
\end{aligned}
$$

d) Para cada $d \in \mathbb{N}$ sea $W_{\mathcal{R}(\mathcal{V}), d}: S \times S \rightarrow\{0,1\}$ dada por:

$$
W_{\mathcal{R}(\mathcal{V}), d}(s, t):= \begin{cases}1 & \text { si } d_{\mathcal{V}}(s, t)=d \\ 0 & \text { c.c. }\end{cases}
$$

e) Sea $D_{n} \in \mathcal{S}(S)$ con $\#\left(D_{n}\right)=n \geq 2$. Sea $X=\left(X_{s}\right)_{s \in D_{n}}$ con $X_{s} \in$ $\mathcal{L}^{2}(\Omega, \mathcal{A}, \mu, \mathbb{R})$. Sea $d \in \mathbb{N}$, llamaremos $d$-índice de Moran de $X$ asociado al sistema de vecindades $\mathcal{V}$ al índice de Moran de $X$ sobre $D_{n}$ asociado a $W_{\mathcal{R}(\mathcal{V}), d}$.

Notemos que este $d$-índice de Moran podría no estar definida para $\operatorname{algunos} d$. 


\subsubsection{Test asintótico de independencia espacial}

Supongamos que existe $d_{S}>0$ tal que

$$
s \in S, t \in S \Rightarrow\|s-t\| \geq d_{S},
$$

siendo || || la norma euclídea de $\mathbb{R}^{2}$.

Sean $\mathcal{V}=\left\{V_{s} / s \in S\right\}$ y $\mathcal{R}$ como al inicio de esta Sección. Sea $R>0$

Sea $W_{\mathcal{R}}: S \times S \rightarrow[0, M]$ con $0<M<\infty$ tal que:

i) $W_{\mathcal{R}}(s, t)=0 \quad \mathrm{si}(s, t) \notin \mathcal{R}, o s=t, o\|s-t\|>R$.

ii) Para cada $s \in S$ se tiene

$$
\sum_{t \in \mathcal{R}_{s}} W_{\mathcal{R}}(s, t) \neq 0,
$$

siendo $\mathcal{R}_{s}:=\left\{t \in S / t \in V_{s}\right\}$.

Supongamos que

$$
\#\left(V_{s}\right) \leq M \forall s \in S \text {. }
$$

Definición 4.6.10. Sea $\emptyset \neq \mathcal{R} \subset\left\{(s, t) \in S \times S / t \in V_{s}\right\}$.

Sea $W_{\mathcal{R}}: S \times S \rightarrow[0, M]$ con $0<M<\infty$

$$
W_{\mathcal{R}}(s, t):= \begin{cases}0 & \text { si } t=s, s \in S \\ 0 & \text { si }(s, t) \notin \mathcal{R} \\ >0 & \text { si }(s, t) \in \mathcal{R} .\end{cases}
$$

Diremos que $W_{\mathcal{R}}$ es una matriz de contigüidad asociada a $R$.

Para cada $n \geq 2$ entero sea $D_{n} \in \mathcal{S}(S)$ con $\#\left(D_{n}\right)=n$.

Sea $X=\left(X_{s}\right)_{s \in S}$ tal que

$$
\begin{aligned}
\mu\left(X_{s}\right) & =\mu_{X} \quad \forall s \in S \\
\mu\left(\left(X_{s}-\mu_{X}\right)^{2}\right) & =\sigma_{X}^{2}<\infty \quad \forall s \in S
\end{aligned}
$$

con $\mu_{X}$ y $\sigma_{X}^{2}>0$ a ser estimados.

Para cada $n=1,2, \ldots$ sea $I_{W_{\mathcal{R}}, D_{n}}^{M}(X)$ como en la Definición 4.6.8.

Teorema 4.6.11. Sean

a) $H_{0}: X=\left(X_{s}\right)_{s \in S}$ está formado por v.a. $\mu$-independientes.

b) $\exists \delta>0$ tal que $\sup _{s \in S} \mu\left(\left|X_{s}\right|^{4+2 \delta}\right)<\infty$.

c) $\underline{\lim } \frac{S_{1, D_{n}}\left(W_{\mathcal{R}}\right)}{n}>0$.

Entonces:

$$
\frac{S_{0, D_{n}}\left(W_{\mathcal{R}}\right)}{\left(S_{1, D_{n}}\left(W_{\mathcal{R}}\right)\right)^{1 / 2}} I_{W_{\mathcal{R}}, D_{n}}^{M}(X) \stackrel{\mathcal{D}}{\rightarrow} \mathcal{N}(0,1) .
$$

Para una demostración ver pag 168 de ([5]). 


\subsubsection{Cálculo exacto de la esperanza y varianza del índice de Moran bajo normalidad}

Definición 4.6.12. Sea $h: \mathbb{R}^{n} \mapsto \mathbb{R}$. Se dice que $h$ es una función homogénea de grado $\beta \geq 0$ real, si

$$
h\left(\lambda x_{1}, \ldots, \lambda x_{n}\right)=\lambda^{\beta} h\left(x_{1}, \ldots, x_{n}\right),
$$

cualesquiera sean $\lambda \in \mathbb{R} \backslash\{0\}$ y $\left(x_{1}, \ldots, x_{n}\right) \in \mathbb{R}^{n}$.

Lema 4.6.13. Sean: $E:=\mathbb{R} \times[0,+\infty)$ con la métrica usual; $\mathcal{E}$ la $\sigma$-álgebra de Borel de $E ; \mu$ y $\nu$ dos probabilidades sobre $(E, \mathcal{E})$. Si

$$
\int_{E} \exp (i u x-v y) \mu(d(x, y))=\int_{E} \exp (i u x-v y) \nu(d(x, y)),
$$

cualesquiera sean $u \in \mathbb{R}$ y $v \geq 0$.Entonces

$$
\mu=\nu .
$$

Demostración. Ejercicio. (Ayuda: Sea

$$
\mathcal{F}_{0}:=\{f: E \rightarrow \mathbb{R} / f \text { es continua y con soporte compacto }\} .
$$

Es bien conocido (ver ejemplo: Parthasarathy (1967)([10]), Theorem 5.9, pag. 39) que:

$$
\int f d \mu=\int f d \nu \quad \forall f \in \mathcal{F}_{0} \Rightarrow \mu=\nu .
$$

Sea

$$
\mathcal{A}:=\{f: E \rightarrow \mathbb{R} / f(x, y)=p(x) q(y) \text { con } p \in \mathcal{P} \text { y } q \in \mathcal{Q}\},
$$

siendo:

$$
\begin{gathered}
\mathcal{P} \quad:=\left\{p: \mathbb{R} \rightarrow \mathbb{C} / p(x)=\sum_{k=1}^{n} a_{k} e^{i u_{k} x}\right. \\
\left.\quad \operatorname{con} n \geq 1, a_{k} \in \mathbb{R}, u_{k} \in \mathbb{R}, k=1, \ldots, n\right\} . \\
\mathcal{Q} \quad:=\left\{q:[0,+\infty) \rightarrow \mathbb{R} / q(x)=\sum_{j=1}^{m} b_{j} e^{-v_{j} x}\right. \\
\\
\text { con } \left.m \geq 1, b_{j} \in \mathbb{R}, v_{j} \geq 0, j=1, \ldots, n\right\} .
\end{gathered}
$$

Por el Teorema de Weierstrass se deduce que $\mathcal{A}$ es denso en $\mathcal{F}_{0}$ con respecto a la topología de la convergencia uniforme sobre compactos).

De aquí se deduce el Lema 4.6.13.

Lema 4.6.14. Sea $Z$ una v.a. con distribución $\chi^{2}$ con n grados de libertad. Entonces:

$$
E\left(e^{-s Z}\right)=\frac{1}{(2 s+1)^{\frac{n}{2}}} \quad \forall s \geq 0 .
$$


Demostración. Ejercicio. Ayuda: es el resultado de un cálculo directo teniendo en cuenta que la densidad de $Z$ es:

$$
f(x)=\frac{1}{\Gamma\left(\frac{n}{2}\right) 2^{\frac{n}{2}}} x^{\frac{n}{2}-1} e^{-\frac{x}{2}} 1_{(0,+\infty)}(x) .
$$

Teorema 4.6.15. Sean: $h: \mathbb{R}^{n} \mapsto \mathbb{R}$ homogénea de grado $0 ; X_{1}, \ldots, X_{n}$ v.a.i.i.d. $\mathcal{N}(0,1)$

$$
Q\left(X_{1}, \ldots, X_{n}\right):=\sum_{j=1}^{n} X_{j}^{2} .
$$

Entonces $h\left(X_{1}, \ldots, X_{n}\right)$ y $Q\left(X_{1}, \ldots, X_{n}\right)$ son independientes.

Demostración. Por el Lema 4.6.13 bastará ver que

$$
\begin{aligned}
& E\left(\exp \left(i u h\left(X_{1}, \ldots, X_{n}\right)-s Q\left(X_{1}, \ldots, X_{n}\right)\right)\right)= \\
& E\left(-s Q\left(X_{1}, \ldots, X_{n}\right)\right) E\left(\exp \left(i u h\left(X_{1}, \ldots, X_{n}\right)\right)\right),
\end{aligned}
$$

$\forall u \in \mathbb{R}$ y $\forall s \geq 0$.

Ahora por el Lema 4.6.14, (1) es equivalente a:

$$
\begin{gathered}
E\left(\exp \left(i u h\left(X_{1}, \ldots, X_{n}\right)-s Q\left(X_{1}, \ldots, X_{n}\right)\right)\right)= \\
\frac{1}{(2 s+1)^{\frac{n}{2}}} E\left(\exp \left(i u h\left(X_{1}, \ldots, X_{n}\right)\right)\right),
\end{gathered}
$$

$\forall u \in \mathbb{R} y \forall s \geq 0$

Veamos entonces que se cumple (2). Pongamos $X:=\left(X_{1}, \ldots, X_{n}\right)$.

Sean: $u \in \mathbb{R}$ y $s \geq 0$ cualesquiera.

$$
\begin{aligned}
E(\exp (i u h(X) & -s Q(X))) \\
& =\frac{1}{(2 \pi)^{n / 2}} \int_{\mathbb{R}^{n}} \exp (i u h(\tilde{x})-s Q(\tilde{x})) \exp \left(-\frac{1}{2} Q(\tilde{x})\right) d \tilde{x}= \\
& =\frac{1}{(2 \pi)^{n / 2}} \int_{\mathbb{R}^{n}} \exp \left(i u h(\tilde{x})-\frac{1}{2}(2 s+1) Q(\tilde{x})\right) d \tilde{x}
\end{aligned}
$$

Para cada $j=1, \ldots, n$ sea $y_{j}:=(1+2 s)^{\frac{1}{2}} x_{j}$. Entonces:

$$
Q(\tilde{y})=(1+2 s) Q(\tilde{x}) \quad \text { y } \quad d \tilde{y}=(1+2 s)^{\frac{n}{2}} d \tilde{x} .
$$

Como $h$ es homogénea de grado 0:

$$
h(\tilde{x})=h(\tilde{y})
$$


Luego:

$$
\begin{aligned}
(3) & =\frac{1}{(2 s+1)^{n / 2}} \frac{1}{(2 \pi)^{n / 2}} \int_{\mathbb{R}_{n}} \exp \left(i u h(\tilde{y})-\frac{1}{2} Q(\tilde{y})\right) d \tilde{y} \\
& =\frac{1}{(2 s+1)^{n / 2}} \int_{\mathbb{R}^{n}} \exp (i u h(\tilde{y}))\left(\frac{1}{(2 \pi)^{n / 2}} \exp \left(-\frac{1}{2} Q(\tilde{y})\right)\right) d \tilde{y} \\
& =\frac{1}{(2 s+1)^{n / 2}} E\left(\exp \left(i u h\left(X_{1}, \ldots, X_{n}\right)\right)\right) .
\end{aligned}
$$

Así, la formula (2) está probada.

Corolario 4.6.16. Sean: $h: \mathbb{R}^{n} \mapsto \mathbb{R}$ homogénea de grado $0 ; X_{1}, \ldots, X_{n}$ v.a.i.i.d. $\mathcal{N}\left(\mu, \sigma^{2}\right)$ con $\mu \in \mathbb{R} y \sigma>0$;

$$
Q\left(X_{1}, \ldots, X_{n}\right):=\sum_{j=1}^{n}\left(X_{j}-\bar{X}\right)^{2},
$$

$\operatorname{con} \overline{X:}=\frac{1}{n} \sum_{j=1}^{n} X_{j}$

Entonces $h\left(X_{1}, \ldots, X_{n}\right)$ y $Q\left(X_{1}, \ldots, X_{n}\right)$ son independientes.

Demostración. Sea $M$ la matriz $n \times n$ dada por:

$$
\begin{aligned}
& M(j, j):=\left(1-\frac{1}{n}\right) \quad \forall j=1, \ldots, n . \\
& M(j, k):=-\frac{1}{n} \quad 1 \leq j, k \leq n, j \neq k .
\end{aligned}
$$

Sea

$$
\tilde{X}=\left(X_{1}, \ldots, X_{n}\right)^{\prime}
$$

Entonces

$$
Q(\tilde{X})=\tilde{X}^{\prime} M \tilde{X}
$$

Por resultados clásicos de Algebra Lineal se tiene que $\exists P$ matriz $n \times n$ ortogonal tal que

$$
P^{\prime} M P=\Lambda
$$

con $\Lambda$ matriz $n \times n$ diagonal dada por:

$$
\begin{aligned}
\Lambda(j, j) & =1 \quad \text { si } 1 \leq j \leq n-1 \\
\Lambda(n, n) & =0 .
\end{aligned}
$$

Sea

$$
\tilde{\eta}=P^{\prime} \tilde{X}
$$


Luego:

$$
Q(\tilde{X})=\tilde{\eta}^{\prime} \Lambda \tilde{\eta}=\sum_{j=1}^{n-1} \eta_{j}^{2}
$$

Por (3), tenemos que:

$$
\tilde{\eta} \sim \mathcal{N}_{n}\left(P^{\prime} \tilde{\theta}, \sigma^{2} I\right)
$$

donde $\tilde{\theta}=(\theta, \ldots, \theta) \in \mathbb{R}^{n}$ e $I=$ matriz identidad $n \times n$.

Es fácil ver que para probar la independencia entre $h\left(X_{1}-\bar{X}, \ldots, X_{n}-\bar{X}\right)$ y $Q(\tilde{X})$ no hay pérdida de generalidad en suponer que $\theta=0$.

Luego, de (4), tenemos:

$$
\tilde{\eta} \sim \mathcal{N}_{n}\left(\tilde{0}, \sigma^{2} I\right)
$$

Por lo tanto

$$
\tilde{\xi}:=\frac{1}{\sigma} \tilde{\eta} \sim \mathcal{N}_{n}(\tilde{0}, I) .
$$

Pongamos

$$
\tilde{\xi}:\left(\xi_{1}, \ldots, \xi_{n}\right)^{\prime} .
$$

Luego: $\xi_{1}, \ldots, \xi_{n-1}$ son v.a.i.i.d. $\mathcal{N}(0,1)$.

Además:

$\left(X_{1}-\bar{X}, \ldots, X_{n}-\bar{X}\right)^{\prime}=M \tilde{X}=P \Lambda P^{\prime} \tilde{X}=P \Lambda \tilde{\eta}=P\left[\begin{array}{c}\eta_{1} \\ \cdots \\ \eta_{n-1} \\ 0\end{array}\right]$

Luego, si $h_{1}: \mathbb{R}^{n-1} \mapsto \mathbb{R}$ está dada por:

$$
h_{1}\left(z_{1}, \ldots, z_{n-1}\right):=h\left(P\left[\begin{array}{c}
z_{1} \\
\cdots \\
z_{n-1} \\
0
\end{array}\right]\right)
$$

se tiene:

$$
h\left(X_{1}-\bar{X}, \ldots, X_{n}-\bar{X}\right)=h_{1}\left(\eta_{1}, \ldots, \eta_{n-1}\right) .
$$

Además, como $h$ es homogénea de grado $0, h_{1}$ también lo es. 
Por consiguiente, como

$$
Q(\tilde{X})=\sum_{j=1}^{n-1} \eta_{j}^{2}
$$

se tiene

$$
h\left(X_{1}-\bar{X}, \ldots, X_{n}-\bar{X}\right)=h_{1}\left(\xi_{1}, \ldots, \xi_{n-1}\right)
$$

y $\sum_{j=1}^{n-1} \xi_{j}^{2}$ son independientes por el Teorema 4.6.15.

De $(7)$ y $(8)$ se deduce ahora fácilmente que $h\left(X_{1}-\bar{X}, \ldots, X_{n}-\bar{X}\right)$ y $Q(\tilde{X})$ son independientes.

Proposición 4.6.17. Continuación de la Definición 4.6.8. Supongamos que $D_{n}=\left\{s_{1}, \ldots, s_{n}\right\}$ y que $X_{s_{1}}, \ldots, X_{s_{n}}$ son v.a.i.i.d. $\mathcal{N}\left(\theta, \sigma^{2}\right)$ con $\theta \in \mathbb{R}$ $y \sigma>0$.

Sea $p \geq 1$ entero. Entonces:

$$
E\left(\left(I_{W_{\mathcal{R}}, D_{n}}^{M}(\tilde{X})\right)^{p}\right)=\frac{n^{p} E\left(\left(C_{W_{\mathcal{R}}, D_{n}}(\tilde{X})\right)^{p}\right)}{\left(S_{0, D_{n}}\left(W_{\mathcal{R}}\right)\right)^{p} E\left(\left(\sum_{s \in D_{n}}\left(X_{s}-\bar{X}\right)^{2}\right)^{p}\right)},
$$

siendo

$$
\begin{aligned}
& \tilde{X} \quad: \quad=\left(X_{1}, \ldots, X_{n}\right)^{\prime} ; \bar{X}:=\frac{1}{n} \sum_{j=1}^{n} X_{j} \\
& I_{W_{\mathcal{R}}, D_{n}}^{M}(\tilde{X}) \quad:=\frac{n C_{W_{\mathcal{R}}, D_{n}}(\tilde{X})}{S_{0, D_{n}}\left(W_{\mathcal{R}}\right) \sum_{s \in D_{n}}\left(X_{s}-\bar{X}\right)^{2}} \\
& C_{W_{\mathcal{R}}, D_{n}}(\tilde{X}):=\sum_{j=1}^{n} \sum_{k=1}^{n} W_{\mathcal{R}}\left(s_{j}, s_{k}\right)\left(X_{s_{j}}-\bar{X}\right)\left(X_{s_{k}}-\bar{X}\right) ; \\
& S_{0, D_{n}}\left(W_{\mathcal{R}}\right):=\sum_{j=1}^{n} \sum_{k=1}^{n} W_{\mathcal{R}}\left(s_{j}, s_{k}\right) .
\end{aligned}
$$

Demostración. Es fácil ver que $I_{W_{\mathcal{R}}, D_{n}}^{M}$ es homogénea de grado 0, considerada como función de $\mathbb{R}^{n}$ en $\mathbb{R}$.

$$
E\left(\left(n C_{W_{\mathcal{R}}, D_{n}}(\tilde{X})\right)^{p}\right)=E\left(\frac{\left(n C_{W_{\mathcal{R}}, D_{n}}(\tilde{X})\right)^{p}}{\left(Q_{W}(\tilde{X})\right)^{p}}\left(Q_{W}(\tilde{X})\right)^{p}\right),
$$

siendo

$$
Q_{W}(\tilde{X}):=S_{0, D_{n}}\left(W_{\mathcal{R}}\right) \sum_{j=1}^{n}\left(X_{s_{j}}-\bar{X}\right)^{2} .
$$


Por el Corolario 4.6.16 tenemos entonces:

$$
\begin{aligned}
(1) & =E\left(\left(I_{W_{\mathcal{R}}, D_{n}}^{M}(\tilde{X})\right)^{p}\left(Q_{W}(\tilde{X})\right)^{p}\right) \\
& =E\left(\left(I_{W_{\mathcal{R}}, D_{n}}^{M}(\tilde{X})\right)^{p}\right) E\left(\left(Q_{W}(\tilde{X})\right)^{p}\right) .
\end{aligned}
$$

De donde se sigue inmediatamente la Proposición 4.6.17.

Proposición 4.6.18. Sean: $n \geq 2$ entero; $X_{1}, \ldots, X_{n}$ v.a.i.i.d. $\mathcal{N}\left(\mu, \sigma^{2}\right)$. Para cada $j=1, \ldots, n$ sea

$$
Z_{j}:=X_{j}-\bar{X}
$$

donde

$$
\bar{X}:=\frac{1}{n} \sum_{k=1}^{n} X_{k}
$$

Entonces

a) $E\left(Z_{j}\right)=0, \quad j=1, \ldots, n$.

b) $E\left(Z_{j}^{2}\right)=\left(1-\frac{1}{n}\right) \sigma^{2}, \quad j=1, \ldots, n$.

c) $E\left(Z_{j} Z_{k}\right)=-\frac{\sigma^{2}}{n}, \quad 1 \leq j, k \leq n, j \neq k$.

d) $E\left(Z_{j}^{2} Z_{k}^{2}\right)=-\frac{n^{2}-2 n+3}{n^{2}} \sigma^{4}, \quad 1 \leq j, k \leq n, j \neq k$.

e) $E\left(Z_{j}^{2} Z_{k} Z_{l}\right)=-\frac{n-3}{n^{2}} \sigma^{4}, \quad 1 \leq j, k, l \leq n, \#(\{j, k, l\})=3$.

f) $E\left(Z_{j} Z_{k} Z_{j_{1}} Z_{j_{2}}\right)=\frac{3}{n^{2}} \sigma^{4}, \quad 1 \leq j, k, j_{1}, k_{1} \leq n, \#\left(\left\{j, k, j_{1}, k_{1}\right\}\right)=4$.

Demostración. Ejercicio.

Teorema 4.6.19. Continuacióm de la Proposición 4.6.17.

Se cumple:

a) $E\left(I_{W_{\mathcal{R}}, D_{n}}^{M}(\tilde{X})\right)=-\frac{1}{n-1}$.

b) $\operatorname{Var}\left(I_{W_{\mathcal{R}}, D_{n}}^{M}(\tilde{X})\right)=\frac{n^{2} S_{1, D_{n}}\left(W_{\mathcal{R}}\right)-n S_{2, D_{n}}\left(W_{\mathcal{R}}\right)+3\left(S_{0, D_{n}}\left(W_{\mathcal{R}}\right)\right)^{2}}{\left(n^{2}-1\right)\left(S_{0, D_{n}}\left(W_{\mathcal{R}}\right)\right)^{2}}-\frac{1}{(n-1)^{2}}$, con: $S_{0, D_{n}}\left(W_{\mathcal{R}}\right):=\sum_{j=1}^{n} \sum_{k=1}^{n} W_{\mathcal{R}}\left(s_{j}, s_{k}\right) ;$

$S_{1, D_{n}}\left(W_{\mathcal{R}}\right):=\sum_{j=1}^{n} \sum_{k=1}^{n}\left(\left(W_{\mathcal{R}}\left(s_{j}, s_{k}\right)\right)^{2}+W_{\mathcal{R}}\left(s_{j}, s_{k}\right) W_{\mathcal{R}}\left(s_{k}, s_{j}\right)\right) ;$

$S_{2, D_{n}}\left(W_{\mathcal{R}}\right):=\sum_{j=1}^{n}\left(W_{\mathcal{R}}\left(s_{j}, *\right)+W_{\mathcal{R}}\left(*, s_{j}\right)\right)^{2}$

$W_{\mathcal{R}}\left(s_{j}, *\right):=\sum_{k=1}^{n} W_{\mathcal{R}}\left(s_{j}, s_{k}\right)$

$W_{\mathcal{R}}\left(*, s_{j}\right):=\sum_{j=1}^{n} W_{\mathcal{R}}\left(s_{j}, s_{k}\right)$. 
Demostración. Para simplificar la notación pongamos $j$ en lugar de $s_{j}$, $\forall j=1, \ldots, n ;$ y $W$ en lugar de $W_{\mathcal{R}} ; S_{0}$ en lugar de $S_{0, D_{n}}\left(W_{\mathcal{R}}\right) ; S_{1}$ en lugar de $S_{1, D_{n}}\left(W_{\mathcal{R}}\right) ; S_{2}$ en lugar de $S_{2, D_{n}}\left(W_{\mathcal{R}}\right)$. También $N$ en lugar de $n C_{W_{\mathcal{R}}, D_{n}}(\tilde{X}) ; \mathcal{D}$ en lugar de $S_{0, D_{n}}\left(W_{\mathcal{R}}\right) \sum_{s \in D_{n}}\left(X_{s}-\bar{X}\right)^{2} ; I$ en lugar de $I_{W_{\mathcal{R}}, D_{n}}^{M}(\tilde{X}) ; Z_{j}:=X_{s_{j}}-\bar{X}$ para $j=1, \ldots, n$.

\section{Prueba de a):}

Por la Proposición 4.6.17

$$
E(I)=\frac{E(N)}{E(\mathcal{D})}
$$

Ahora:

$$
E(N)=E\left(n \sum_{j=1}^{n} \sum_{k=1}^{n} W_{\mathcal{R}}(j, k) Z_{j} Z_{k}\right)
$$

Como

$$
W(j, j)=0 \quad \forall j=1, \ldots, n,
$$

basta ver qué vale $E\left(Z_{j} Z_{k}\right)$ para $1 \leq j, k \leq n, j \neq k$.

Por la Proposición 4.6.18 tenemos entonces:

$$
(2)=n \sum_{j=1}^{n} \sum_{k=1}^{n} W_{\mathcal{R}}(j, k)\left(-\frac{\sigma^{2}}{n}\right)=-\sigma^{2} S_{0}
$$

Esto es

$$
E(N)=-\sigma^{2} S_{0}
$$

Ahora

$$
E(\mathcal{D})=S_{0} \sum_{j=1}^{n} E\left(Z_{j}^{2}\right)=n S_{0}\left(1-\frac{1}{n}\right) \sigma^{2}=S_{0}(n-1) \sigma^{2} .
$$

De aquí y por (3) y (1) tenemos que

$$
E(I)=-\frac{1}{n-1} .
$$

\section{$\underline{\text { Prueba de b): }}$}

Por lo probado en a)

$$
\operatorname{Var}(I)=E\left(I^{2}\right)-\frac{1}{(n-1)^{2}} .
$$


Por la Proposición 4.6.17 tenemos:

$$
E\left(I^{2}\right)=\frac{E\left(N^{2}\right)}{E\left(\mathcal{D}^{2}\right)} .
$$

Ahora:

$$
\begin{aligned}
E\left(\mathcal{D}^{2}\right) & =S_{0}^{2} E\left(\left(\sum_{j=1}^{n} Z_{j}^{2}\right)^{2}\right)=S_{0}^{2}\left(\operatorname{Var}\left(\sum_{j=1}^{n} Z_{j}^{2}\right)+\left(E\left(\sum_{j=1}^{n} Z_{j}^{2}\right)\right)^{2}\right) \\
& =S_{0}^{2} \cdot\left(\operatorname{Var}\left(\sum_{j=1}^{n} Z_{j}^{2}\right)+(n-1)^{2} \sigma^{2}\right),
\end{aligned}
$$

por lo ya probado en a).

Por otra parte

$$
\sum_{j=1}^{n} Z_{j}^{2} \sim \sigma^{2} \chi_{n-1}^{2} \Rightarrow \operatorname{Var}\left(\sum_{j=1}^{n} Z_{j}^{2}\right)=2(n-1) \sigma^{4} .
$$

Por lo tanto:

$$
(4)=\sigma^{4} S_{0}^{2}\left(2(n-1)+(n-1)^{2}\right)=\sigma^{4} S_{0}^{2}\left(n^{2}-1\right) .
$$

Queda así probado:

$$
E(\mathcal{D})=\sigma^{4} S_{0}^{2}\left(n^{2}-1\right)
$$

Por otra parte:

$$
E\left(N^{2}\right)=n^{2} \sum_{\left((j, k),\left(j_{1}, k_{1}\right)\right) \in T \times T} W(j, k) W\left(j_{1}, k_{1}\right) E\left(Z_{j} Z_{k} Z_{j_{1}} Z_{k_{1}}\right)
$$

siendo:

$$
T:=\{(j, k) / 1 \leq j, k \leq n, j \neq k\} .
$$

Sean ahora:

$$
\begin{gathered}
\mathbb{T}_{1}:=\left\{\left((j, k),\left(j_{1}, k_{1}\right)\right) \in T \times T / j=j_{1} \wedge k=k_{1}\right\} \\
\cup\left\{\left((j, k),\left(j_{1}, k_{1}\right)\right) \in T \times T / j=k_{1} \wedge k=j_{1}\right\} . \\
\mathbb{T}_{2}:=\left\{\left((j, k),\left(j_{1}, k_{1}\right)\right) \in T \times T / j=j_{1} \wedge k \neq k_{1}\right\} \\
\cup\left\{\left((j, k),\left(j_{1}, k_{1}\right)\right) \in T \times T / j \neq j_{1} \wedge k=k_{1}\right\} \\
\cup\left\{\left((j, k),\left(j_{1}, k_{1}\right)\right) \in T \times T / j \neq k_{1} \wedge k=j_{1}\right\} \\
\cup\left\{\left((j, k),\left(j_{1}, k_{1}\right)\right) \in T \times T / j=k_{1} \wedge k \neq j_{1}\right\} .
\end{gathered}
$$




$$
\mathbb{T}_{3}:=\left\{\left((j, k),\left(j_{1}, k_{1}\right)\right) \in T \times T / \#\left(\left\{j, k, j_{1}, k_{1}\right\}\right)=4\right\} .
$$

Por (8) y por la Proposición 4.6.18 tenemos:

$$
\begin{aligned}
& E\left(N^{2}\right)=n^{2}\left[\left(\sum_{(j, k) \in T}(W(j, k))^{2}+\sum_{(j, k) \in T} W(j, k) W(k, j)\right) \frac{n^{2}-2 n+3}{n^{2}} \sigma^{4}\right. \\
& -\frac{(n-3) \sigma^{4}}{n^{2}} \sum_{j=1}^{n} \sum_{k=1}^{n} \sum_{k_{1} \neq k}\left(W(j, k) W\left(j, k_{1}\right)+W(j, k) W\left(k_{1}, k\right)\right. \\
& \left.+W(j, k) W\left(k, k_{1}\right)+W(j, k) W\left(k_{1}, j\right)\right) \\
& \left.+\frac{3}{n^{2}} \sigma^{4} \sum_{\left((j, k),\left(j_{1}, k_{1}\right)\right) \in \mathbb{T}_{3}} W(j, k) W\left(j_{1}, k_{1}\right)\right] \\
& =n^{2} \sigma^{4} S_{1}+3 \sigma^{4} S_{0}^{2}-n \sigma^{4} \sum_{j=1}^{n}\left[2 \sum_{k=1}^{n}(W(j, k))^{2}+2 \sum_{k=1}^{n} W(j, k) W(k, j)\right. \\
& +\sum_{k=1}^{n} \sum_{k_{1} \neq k} W(j, k) W\left(j, k_{1}\right)+\sum_{k=1}^{n} \sum_{k_{1} \neq j} W(j, k) W\left(k_{1}, k\right) \\
& \left.+\sum_{k=1}^{n} \sum_{k_{1} \neq k} W(j, k) W\left(k, k_{1}\right)+\sum_{k=1}^{n} \sum_{k_{1} \neq k} W(j, k) W\left(k_{1}, j\right)\right]
\end{aligned}
$$

último sumando tenemos:

$$
\begin{aligned}
\sum_{j=1}^{n}[] & =2 \sum_{j=1}^{n} \sum_{k=1}^{n}(W(j, k))^{2}+\sum_{j=1}^{n} \sum_{k=1}^{n} \sum_{k_{1} \neq k} W(j, k) W\left(j, k_{1}\right) \\
& +\sum_{j=1}^{n} \sum_{k=1}^{n} \sum_{k_{1} \neq k} W(j, k) W\left(k_{1}, k\right) \\
& \left.+2 \sum_{j=1}^{n} \sum_{k=1}^{n} \sum_{k_{1} \neq k} W(j, k) W\left(k_{1}, j\right)+2 \sum_{j=1}^{n} \sum_{k=1}^{n} W(j, k) W(k, j)\right] \\
& =\sum_{j=1}^{n}\left(\sum_{k=1}^{n} W(j, k)+\sum_{k=1}^{n} W(k, j)\right)^{2}=S_{2} .
\end{aligned}
$$

Luego, por (9)

$$
E\left(N^{2}\right)=n^{2} \sigma^{4} S_{1}+3 \sigma^{4} S_{0}^{2}-n \sigma^{4} S_{2} .
$$

De aquí, por (7) y (3) tenemos

$$
E\left(I^{2}\right)=\frac{n^{2} S_{1}+3 S_{0}^{2}-n S_{2}}{S_{0}^{2}\left(n^{2}-1\right)} .
$$

Por (4), el Teorema 4.6.19 queda demostrado. 


\subsection{4 Índice de Geary}

Continuamos con la misma notación usada al tratar el índice de Moran.

Definición 4.6.20. Sea $X=\left(X_{s}\right)_{s \in \mathcal{D}_{n}}$ con $X_{s} \in \mathcal{L}^{2}(\Omega, \mathcal{A}, \mu, \mathbb{R})$ y $W_{\mathcal{R}}: S \times S \rightarrow[0, M]$ con $0<M<\infty$. Se llama índice de Geary de $X$ (sobre $D_{n}$ ) asociado a $W_{\mathcal{R}}$ a:

$$
I_{W_{\mathcal{R}}, D_{n}}^{G}(X):=\frac{(n-1) \sum_{s \in D_{n}} \sum_{t \in D_{n}} W_{\mathcal{R}}(s, t)\left(X_{s}-X_{t}\right)^{2}}{2 S_{0, D_{n}}\left(W_{\mathcal{R}}\right) \sum_{s \in D_{n}}\left(X_{s}-\bar{X}\right)^{2}},
$$

con

$$
\begin{aligned}
S_{0, D_{n}}\left(W_{\mathcal{R}}\right) & :=\sum_{s \in D_{n}} \sum_{t \in D_{n}} W_{\mathcal{R}}(s, t) y \\
\bar{X} \quad: & =\frac{1}{n} \sum_{s \in D_{n}} X_{s} .
\end{aligned}
$$

Sean: $S_{0, D_{n}}\left(W_{\mathcal{R}}\right)$ como arriba;

$$
\begin{aligned}
& S_{1, D_{n}}\left(W_{\mathcal{R}}\right):=\sum_{s \in D_{n}} \sum_{t \in D_{n}}\left(\left(W_{\mathcal{R}}(s, t)\right)^{2}+W_{\mathcal{R}}(s, t) W_{\mathcal{R}}(t, s)\right) \mathrm{y} \\
& S_{2, D_{n}}\left(W_{\mathcal{R}}\right):=\sum_{s \in D_{n}}\left(\sum_{t \in D_{n}} W_{\mathcal{R}}(s, t)+\sum_{t \in D_{n}} W_{\mathcal{R}}(t, s)\right)^{2} .
\end{aligned}
$$

Teorema 4.6.21. Sean a), b) y c) como en el Teorema 4.6.11. Entonces:

$$
\left(\frac{S_{0, D_{n}}\left(W_{\mathcal{R}}\right)}{2 S_{1, D_{n}}\left(W_{\mathcal{R}}\right)+S_{2, D_{n}}\left(W_{\mathcal{R}}\right)}\right)^{1 / 2}\left(I_{W_{\mathcal{R}}, D_{n}}^{G}(X)-1\right) \stackrel{\mathcal{D}}{\rightarrow} \mathcal{N}(0,1) .
$$

Demostración. Ver lo dicho para la demostración del Teorema 4.6.11.

\subsubsection{Cálculo exacto de la esperanza y varianza del índice de Geary bajo normalidad}

Sea $n \geq 2$. A los fines de simplificar la notación pongamos:

$$
\begin{aligned}
& \mathcal{D}_{n}:=\{1, \ldots, n\} ; W=W_{\mathcal{R}} ; \\
& S_{0}:=S_{0, D_{n}}\left(W_{\mathcal{R}}\right) ; S_{1}=S_{1, D_{n}}\left(W_{\mathcal{R}}\right) ; S_{2}=S_{2, D_{n}}\left(W_{\mathcal{R}}\right) ; \\
& I_{G}=I_{W_{\mathcal{R}}, D_{n}}^{G}(X) .
\end{aligned}
$$

Es fácil ver que $I_{G}$ como función de $\mathbb{R}^{n}$ es homogénea de grado 0 (con $\left.I_{G}(x, \ldots, x):=0 \forall x \in \mathbb{R}\right)$; tenemos entonces un resultado análogo al de la Proposición 4.6.17: 
Proposición 4.6.22. Si $X_{1}, \ldots, X_{n}$ son v.a.i.i.d. $\mathcal{N}\left(\theta, \sigma^{2}\right)$ con $\theta \in \mathbb{R} y$ $\sigma>0$.

Sea $p \geq 1$ entero. Entonces:

$$
E\left(\left(I_{G}\right)^{p}\right)=\frac{(n-1)^{p} E\left(\left(\sum_{j=1}^{n} \sum_{k=1}^{n} W(j, k)\left(X_{j}-X_{k}\right)^{2}\right)^{p}\right)}{2^{p} S_{0} p E\left(\left(\sum_{j=1}^{n}\left(X_{j}-\bar{X}\right)^{2}\right)^{p}\right)} .
$$

Demostración. Ejercicio.

Teorema 4.6.23. Si $X_{1}, \ldots, X_{n}$ son v.a.i.i.d. $\mathcal{N}\left(\theta, \sigma^{2}\right) \operatorname{con} \theta \in \mathbb{R} y \sigma>0$ se tiene:

a) $E\left(I_{G}\right)=1$.

b) $\operatorname{Var}\left(I_{G}\right)=\frac{\left(2 S_{1}+S_{2}\right)(n-1)-4 S_{2}}{2(n+1) S_{0}}$.

Demostración. Ejercicio. 



\section{Bibliografía}

[1] Adler, R.J. (1981). The Geometry of Random Fields. Wiley.

[2] Besag, J., 1989, Towards Bayesian image analysis. Journal of Applied Statistics, 16, pp. 395-407.

[3] Bustos, O. y Ojeda S. (1994). Campos Aleatorios Markovianos en Procesamiento de Imágenes. Trabajos de Matemática, Serie "B" 25/94, Facultad de Matemática, Astronomía y Física, Universidad Nacional de Córdoba.

[4] Frery, A.C. Ferrero, S., and Bustos, O.H. (2009). The Influence of training errors, context and number of bands in the accuracy of image classification. International Journal of Remote Sensing. 30, 6: 14251440

[5] Gaetan, C. and Guyon, X. (2010). Spatial Statistics and Modeling. Springer.

[6] Georgii, H. (1988). Gibbs Measures and Phase Transitions. Walter de Gruyter, Berlin.

[7] Guyon, X. (1995). Random Fields on a Network: Modeling, Statistics and Applications. Springer.

[8] Halmos, P. (1974). Measure Theory. Springer.

[9] Ojeda, S., Vallejos, R. and Bustos, O. (2010). A new image segmentation algorithm with applications to image inpainting. Computational Statistics and Data Analysis 54 (2010) 2082-2093.

[10] Parthasarathy, K.R. (1967). Probability Measures on Metric Spaces. Academic Press. 
[11] Schlather, M. (1999). Introduction to positive definite functions and to unconditional simulation of random fields. Tech. Rep. ST 99-10, Lancaster University, Lancaster.

Oscar Humberto Bustos

Consejo de Investigaciones en Matemática (CONICET)

Facultad de Matemática, Astronomía y Física - Universidad Nacional de Córdoba

Haya de la Torre y Medina Allende, Ciudad Universitaria

Córdoba

e-mail: bustos@famaf.unc.edu.ar

Aureliano Andrés Guerrero

Consejo de Investigaciones en Matemática (CONICET)

Facultad de Matemática, Astronomía y Física - Universidad Nacional de Córdoba

Haya de la Torre y Medina Allende, Ciudad Universitaria

Córdoba

e-mail: aguerrero@famaf.unc.edu.ar 


\section{Índice alfabético}

Anisotropía, 22

Anisotropía Geométrica, 22

Automodelo

de Besag, 73

Binomial, 78

de Besag Generalizado, 73

Exponencial, 81

Logístico, 78

Poisson, 79

Automodelos

$\mathcal{G}$-markovianos, 77

Campos Aleatorios de Gibbs Markov, 48

Continuidad y Diferenciabilidad, 23 caso Estacionario, 24

Correlación Isotrópica, 16

d-indice de Moran, 97

derivada espectral de $C^{0}, 26$

Descomposición de Cholesky, 40

Distribución de Gibbs asociada, 51

Ejemplos de Covarianza y Variogramas, 21

Especificación $\mathcal{G}$-markoviana, 51

Especificación Inducida, 50

Estacionaridad Estricta. Isotropía, 16

Estimación del Semivariograma con Presencia de Covariables, 90

Estimación en Geoestadística, 82

Caso Isotrópico, 86

Estimador de Máxima

Verosimilitud, 91
Estimador Empírico del

Semivariograma Isotrópico

Caso Paramétrico, 87

Estimador Empírico del Semi-

variograma Isotrópico, 86

Fórmula de Möbius, 65

Función Semivariograma

Estimador de Mínimo Contraste, 89

Estimador de Mínimos Cuadrados, 89

Función

condicionalmente definida, 19

de Bessel modificada, 21

Incremento, 19

localmente acotada, 19

Oscilación en el infinito, 67

función

local, 59

quasilocal, 59

Función

Semivariograma, 18

Función Semivariograma

Direccional, 22

Distribución del Estimador Empírico de la

Procesos Gaussianos, 83

Estimador de Mínimos Cuadrados Generalizado, 89

Estimador Empírico de la, 83

Isotrópicos, 21

Meseta de, 20

Modelos, 21

Efecto Pepita Puro, 21 
Esférico, 21

Exponencial, 21

Exponencial Generalizado, 21

Gaussiano, 21

Matérn, 21

Potencial, 22

Rango de, 20

Rango práctico de, 20

$\mathcal{G}$-Potencial, 53

Geary, índice de, 108

Cálculo Exacto de la Esperanza y la Varianza bajo, 108

grafo asociado

a la representación CAR, 42

a la representación SAR, 43

Imagen, 10

Independencia Espacial

Test Asintótico, 98

Inferencias en Modelos Espaciales, 82

Kriging Simple, 46

Kriging Universal, 47

Matriz de Contigüidad, 95, 98

Matriz de Contigüidad

Normalizada, 95

Modelo de Ising, 55-57

Modelo de Potts-Strauss, 58

Modelo de Regresión Espacial, 44

Análisis de la covarianza, 45

Análisis de la varianza, 45

Ejemplo: Dependencia exógena, 45

Ejemplo: Superficie de Respuesta, 44

Modelos ARMA, 28

Modelos Autoregresivos Espaciales, 28

Modelo ARMA, 28

Modelos Autorregresivos no sobre redes finitas, 39
Grafo asociado a modelo SAR, 43

Proceso Gauss-Markov, 41

Representación CAR-Markov, 41

Modelos Espaciales de Segundo Orden, 10

Moran, índice de, 96

Cálculo Exacto de la Esperanza y la Varianza bajo, 99

Movimiento Browniano, 17

Orden Lexicográfico, 31, 86

Potencial, 49

a-normalizado, 59

Acotado, 51

de Rango Finito, 61

Energía del, 49

Equivalencia entre, 62

Función partición, 50

Invariante por traslaciones, 72

Normalizado e Identificable, 59

$\lambda$-admisible, 49

sobre pares, 73

Sumable, 52

Uniformemente Convergente, 62

Potencial $\Phi^{a}, 70$

Potenciales y distribuciones de Gibbs, 49

Predicción con Varianza Conocida, 46

Predictor, 32

Optimo, 33

Predictor Lineal, 32

Optimo, 32

Predictor Lineal Optimo Varianza Conocida, 46

proceso de medias móviles

de orden finito, 37

de orden infinito, 28, 37

Proceso de segundo orden

Continuo en Media

Cuadrática, 23 
Diferenciable en Media

Cuadrática, 23

Proceso Estocástico de Imágenes, 11

P-independientes, 11

Coeficiente de Correlación de, 12

Covarianza de, 12

Débilmente Estacionario de primer orden, 12

Débilmente Estacionarios de segundo orden, 12

de primer orden, 11

de segundo orden, 11

Estrictamente Estacionario, 16

Gaussiano, 12

Intrínsecamente Estacionario, 18

Varianza de, 11

Proceso Intrínseco

Anisotrópico, 22

de Media Cuadrática de orden 1,25

de Media Cuadrática de orden $\mathrm{m}, 25$

de Media Cuadrática Infinitamente, 26

Isotrópico, 22

proceso L-markoviano (CAR(L)), 32

Procesos Autorregresivos

Condicionales Estacionarios, 31

Procesos Intrínsecos y Variogramas, 18

Procesos SAR, 29

Isotrópico, 30

Procesos SAR(1), 30

Representación AR, 40

Representación ARMA, 40

Representación CAR asociada al SARlocal uniparamétrico, 43

Representación CAR-Markov, 41

Representación Espectral de

caso $S=\mathbb{R}, 27$

caso $S=\mathbb{Z}^{2}, 27$
Representación Espectral de

Covarianzas

caso $S=\mathbb{R}^{2}, 26$

Representación MA, 40

Representación SAR-local uniparamétrica, 40

Representación SAR-local uniparamétrica, 40

Ruido Blanco, 16 en sentido débil, 16 en sentido fuerte, 16

SAR causal, 37

SAR factorizante, 39

SAR no causal, 38

Sistema de Vecindades, 42, 51

Trayectoria, 11, 23

Casi Seguramente Continuas (c.s.c.), 23

Validación Cruzada, 91

Validación de Modelos para Semivariogramas Isotrópicos, 91

Validación por "bootstrap" paramétrico, 93

Variogramas para Procesos, 20 